\title{
Subgradients of Marginal Functions in Parametric Control Problems of Partial Differential Equations
}

\author{
Nguyen Thanh Qui* and Daniel Wachsmuth ${ }^{\dagger}$ \\ July 17, 2018
}

\begin{abstract}
The paper studies generalized differentiability properties of the marginal function of parametric optimal control problems of semilinear elliptic partial differential equations. We establish upper estimates for the regular and the limiting subgradients of the marginal function. With some additional assumptions, we show that the solution map of the perturbed optimal control problems has local upper Hölderian selections. This leads to a lower estimate for the regular subdifferential of the marginal function.
\end{abstract}

Key words. Perturbed control problem, semilinear elliptic equation, marginal function, local upper Hölderian selection, regular subgradient, limiting subgradient.

AMS subject classifications. 35J61, 49J52, 49J53, 49K20, 49K30.

\section{Introduction}

It is well recognized that optimal value function (or, marginal function) and solution map of parametric optimization problems are very important in variational analysis, optimization theory, control theory, etc. Problems of investigation on generalized differentiability properties of the marginal function and the solution map of parametric optimization problems are in the research direction of differential stability of optimization problems. Many researchers have had contributions to this research direction such as Aubin [5], Auslender [6], Bonnans and Shapiro [8], Dien and Yen [13], Gauvin and Dubeau [14, 15], Gollan [16], Mordukhovich et al. [20, 21], Rockafellar [25], Thibault [27]. In general, marginal functions are complicated and intrinsically nonsmooth in perturbed parameters, therefore generalized differentiability properties of marginal functions play a crucial role in order to derive important information on sensitivity and stability of optimization problems.

Recently, Mordukhovich et al. [21] derived formulas for computing and estimating the regular subdifferential and the limiting (Mordukhovich, singular) subdifferentials of marginal functions in Banach spaces and specified these results for important classes of problems in parametric optimization with smooth and nonsmooth data. Motivated by the results of [21], some new results on differential stability of convex optimization problems under inclusion constraints as well as under Aubin's regularity condition have been provided in [2, 3]. In addition, differential stability of parametric optimal control problems governed by

${ }^{*}$ College of Information and Communication Technology, Can Tho University, Campus II, $3 / 2$ Street, Can Tho, Vietnam; ntqui@cit.ctu.edu.vn. Institut für Mathematik, Universität Würzburg, Emil-Fischer-Str. 30, 97074 Würzburg, Germany; thanhqui.nguyen@mathematik.uni-wuerzburg.de. The research of this author was supported by the Alexander von Humboldt Foundation.

†Institut für Mathematik, Universität Würzburg, Emil-Fischer-Str. 30, 97074 Würzburg, Germany; daniel.wachsmuth@mathematik.uni-wuerzburg.de. 
ordinary differential equations (ODEs) has been studied by many authors in [1, 11, 28, 29, 30, where many results on the first-order behavior of the marginal function of parametric continuous/discrete optimal control problems with linear constraints, convex/nonconvex cost functionals have been established.

To the best of our knowledge, although there were many works on differential stability of optimal control problems of ODEs, the problem of study on differential stability of optimal control problems governed by partial differential equations (PDEs) remains open. For this reason, in the present paper we focus on the study of generalized differentiability properties of the marginal function of perturbed optimal control problems for PDEs. Namely, we will establish new formulas for computing/estimating the regular subdifferential as well as the limiting subdifferentials in the Mordukhovich's sense of the marginal function of perturbed optimal control problems of semilinear elliptic PDEs with control constraints.

For the original control problem in question, we are interested in two classes of perturbed control problems with respect to two different parametric spaces. In the first class of perturbed problems, under some standard assumptions posed on the initial data of the original control problem, we establish new upper estimates for the regular subdifferential, the Mordukhovich subdifferential, and the singular subdifferential of the marginal function of the perturbed control problems. In addition, these upper estimates for the regular and the Mordukhovich subdifferentials of the marginal function will hold as equalities provided that the solution map of the perturbed control problems has a local upper Lipschitzian selection at the reference point in the graph of the solution map. For the second class of perturbed problems, we consider parametric bang-bang control problems, where the cost functional of such control problems does not involve the usual quadratic term for the control. With some additional assumptions to the above standard assumptions, we show that the solution map of the perturbed control problems admits a local upper Hölderian selection at the reference point in the graph of the solution map. This leads to a new lower estimate for the regular subdifferential of the marginal function.

The rest of the paper is organized as follows. A class of optimal control problems together with standard assumptions in optimal control theory of PDEs and auxiliary results are stated in Section 2. In Section 3, we establish upper estimates for the regular, the Mordukhovich, and the singular subdifferentials of the marginal function to the first class of perturbed control problems. Section 4 is devoted to prove the existence of local upper Hölderian selections of the solution map and new lower estimates for the regular subdifferential of the marginal function to the second class of perturbed control problems (parametric bang-bang control problems). Some concluding remarks and open problems are provided in the last section.

\section{Preliminaries}

\subsection{Control problem statement}

The original optimal control problem that we are interested in this paper is stated as follows

$$
\begin{cases}\text { Minimize } & J(u)=\int_{\Omega} L\left(x, y_{u}(x)\right) d x+\frac{1}{2} \int_{\Omega} \zeta(x) u(x)^{2} d x \\ \text { subject to } & \alpha(x) \leq u(x) \leq \beta(x) \quad \text { for a.a. } x \in \Omega,\end{cases}
$$

where $\zeta \in L^{2}(\Omega)$ with $\zeta(x) \geq 0$ for a.a. $x \in \Omega$, and $y_{u}$ is the weak solution of the following Dirichlet problem

$$
\left\{\begin{aligned}
A y+f(x, y) & =u & & \text { in } \Omega \\
y & =0 & & \text { on } \Gamma,
\end{aligned}\right.
$$


where the letter $A$ denotes the second-order elliptic differential operator of the form

$$
A y(x)=-\sum_{i, j=1}^{N} \partial_{x_{j}}\left(a_{i j}(x) \partial_{x_{i}} y(x)\right) .
$$

The corresponding perturbed control problem of (2.1) is given below

$$
\begin{cases}\text { Minimize } & \mathcal{J}(u, e)=J\left(u+e_{y}\right)+\left(e_{J}, y_{u+e_{y}}\right)_{L^{2}(\Omega)} \\ \text { subject to } & u \in \mathcal{G}(e)=\mathcal{U}_{a d}(e) \cap \mathcal{Q}\end{cases}
$$

where $J(\cdot)$ is the cost functional of problem (2.1),$y_{u+e_{y}}$ is the weak solution of the perturbed Dirichlet problem

$$
\left\{\begin{aligned}
A y+f(x, y) & =u+e_{y} & & \text { in } \Omega \\
y & =0 & & \text { on } \Gamma,
\end{aligned}\right.
$$

$\mathcal{Q}$ is a given subset of $L^{p_{0}}(\Omega)$, and

$$
\mathcal{U}_{a d}(e)=\left\{u \in L^{q_{0}}(\Omega) \mid\left(\alpha+e_{\alpha}\right)(x) \leq u(x) \leq\left(\beta+e_{\beta}\right)(x) \text { for a.a. } x \in \Omega\right\} .
$$

We introduce $E=L^{p_{1}}(\Omega) \times L^{p_{2}}(\Omega) \times L^{p_{3}}(\Omega) \times L^{p_{4}}(\Omega)$ the parametric space with the norm of $e=\left(e_{J}, e_{y}, e_{\alpha}, e_{\beta}\right) \in E$ given by

$$
\|e\|_{E}=\left\|e_{J}\right\|_{L^{p_{1}(\Omega)}}+\left\|e_{y}\right\|_{L^{p_{2}(\Omega)}}+\left\|e_{\alpha}\right\|_{L^{p_{3}(\Omega)}}+\left\|e_{\beta}\right\|_{L^{p_{4}}(\Omega)} .
$$

In what follows, we will write $\mathcal{U}_{a d}$ for $\mathcal{U}_{a d}(0)$ the set of admissible controls of problem (2.1).

Let us recall definitions of the marginal function and the solution map of the perturbed control problem (2.3). The marginal function $\mu: E \rightarrow \overline{\mathbb{R}}$ of the perturbed problem (2.3) is defined by

$$
\mu(e)=\inf _{u \in \mathcal{G}(e)} \mathcal{J}(u, e),
$$

and the solution/argminimum map $S: E \rightrightarrows L^{s_{0}}(\Omega)$ of problem (2.3) is given by

$$
S(e)=\{u \in \mathcal{G}(e) \mid \mu(e)=\mathcal{J}(u, e)\} .
$$

The main goal of this paper is to establish explicit formulas for computing/estimating the regular subdifferential, the Mordukhovich subdifferential, and the singular subdifferential of the marginal function $\mu(\cdot)$ in (2.7) at a given parameter $\bar{e} \in E$.

\subsection{Generalized differentiation from variational analysis}

Let us recall some material on generalized differentiation taken from [18. Unless otherwise stated, every reference norm in a product normed space is the sum norm. Given a point $u$ in a Banach space $X$ and $\rho>0$, we denote $B_{\rho}(u)$ the open ball of center $u$ and radius $\rho$ in $X$, and $\bar{B}_{\rho}(u)$ is the corresponding closed ball. In particular, for any $p \in[1, \infty]$, the notation $\bar{B}_{\rho}^{p}(u)$ stands for the closed ball $\bar{B}_{\rho}(u)$ in the space $L^{p}(\Omega)$, i.e.,

$$
\bar{B}_{\rho}^{p}(u)=\left\{v \in L^{p}(\Omega) \mid\|v-u\|_{L^{p}(\Omega)} \leq \rho\right\} .
$$

Let $F: X \rightrightarrows W$ be a multifunction between Banach spaces. The graph and the domain of $F$ are the sets $g p h F:=\{(u, v) \in X \times W \mid v \in F(u)\}$ and $\operatorname{dom} F:=\{u \in X \mid F(u) \neq \emptyset\}$, respectively. We say that $F$ is locally closed around the point $\bar{\omega}=(\bar{u}, \bar{v}) \in \operatorname{gph} F$ if gph $F$ is locally closed around $\bar{\omega}$, i.e., there exists a closed ball $\bar{B}_{\rho}(\bar{\omega})$ such that $\bar{B}_{\rho}(\bar{\omega}) \cap \operatorname{gph} F$ is closed in $X \times W$. 
For a multifunction $\Phi: X \rightrightarrows X^{*}$, the sequential Painlevé-Kuratowski upper limit of $\Phi$ as $u \rightarrow \bar{u}$ is defined by

$$
\begin{aligned}
\underset{u \rightarrow \bar{u}}{\operatorname{Limsup}} \Phi(u)=\left\{u^{*} \in X^{*} \mid \text { there exist } u_{n} \rightarrow \bar{u} \text { and } u_{n}^{*} \stackrel{w^{*}}{\longrightarrow} u^{*}\right. \text { with } \\
\\
\left.u_{n}^{*} \in \Phi\left(u_{n}\right) \text { for every } k \in \mathbb{N}=\{1,2, \ldots\}\right\} .
\end{aligned}
$$

Given an extended-real-valued function $\phi: X \rightarrow \overline{\mathbb{R}}$ and $\bar{u} \in \operatorname{dom} \phi:=\{u \in X \mid \phi(u)<\infty\}$, the regular subdifferential (also called the Fréchet subdifferential) of $\phi$ at the point $\bar{u}$ is the set $\widehat{\partial} \phi(\bar{u}):=\widehat{\partial}_{0} \phi(\bar{u})$, where $\widehat{\partial}_{\varepsilon} \phi(\bar{u})$ with $\varepsilon \geq 0$ is the collection of $\varepsilon$-subgradients of $\phi$ at $\bar{u}$ defined by

$$
\widehat{\partial}_{\varepsilon} \phi(\bar{u})=\left\{u^{*} \in X^{*} \mid \liminf _{u \rightarrow \bar{u}} \frac{\phi(u)-\phi(\bar{u})-\left\langle u^{*}, u-\bar{u}\right\rangle}{\|u-\bar{u}\|} \geq-\varepsilon\right\},
$$

and the regular/Fréchet upper subdifferential of $\phi$ at $\bar{u}$ is given by

$$
\widehat{\partial}^{+} \phi(\bar{u})=-\widehat{\partial}(-\phi)(\bar{u}) .
$$

The limiting basic subdifferential (the Mordukhovich subdifferential) of $\phi$ at $\bar{u}$ is defined via the sequential outer limit (2.9) by

$$
\partial \phi(\bar{u})=\underset{u \underset{\varepsilon \downarrow 0}{\operatorname{Limsup}} \bar{u}}{ } \widehat{\partial}_{\varepsilon} \phi(u),
$$

and the limiting singular subdifferential (the singular subdifferential for short) of $\phi$ at $\bar{u}$ is given by

$$
\begin{aligned}
& \partial^{\infty} \phi(\bar{u})=\operatorname{Limsup} \lambda \widehat{\partial}_{\varepsilon} \phi(u), \\
& \underset{\varepsilon, \lambda \downarrow 0}{\stackrel{\phi}{\longrightarrow} \bar{u}}
\end{aligned}
$$

where the notation $u \stackrel{\phi}{\rightarrow} \bar{u}$ means that $u \rightarrow \bar{u}$ with $\phi(u) \rightarrow \phi(\bar{u})$.

Note that we can equivalently put $\varepsilon=0$ in (2.12) and (2.13) if $X$ is an Asplund space [4] (see also [18, 22 for more details) and $\phi$ is lower semicontinuous around $\bar{u}$. It is obvious that $\widehat{\partial} \phi(\bar{u}) \subset \partial \phi(\bar{u})$ whenever $\phi(\bar{u})$ is finite. If the latter inclusion holds as equality, $\phi$ is said to be lower regular at $\bar{u}$. The class of lower regular functions is sufficiently large and important in variational analysis and optimization; see [18, 19, 26] for more details and applications.

Given a nonempty set $\Theta \subset X$, the regular and Mordukhovich normal cones to $\Theta$ at $\bar{u} \in \Theta$ are respectively defined by

$$
\widehat{N}(\bar{u} ; \Theta)=\widehat{\partial} \delta(\bar{u} ; \Theta) \quad \text { and } \quad N(\bar{u} ; \Theta)=\partial \delta(\bar{u} ; \Theta),
$$

where $\delta(\cdot ; \Theta)$ is the indicator function of $\Theta$ defined by $\delta(u ; \Theta)=0$ for $u \in \Theta$ and $\delta(u ; \Theta)=\infty$ otherwise. If $X$ is an Asplund space and $\Theta \subset X$ is locally closed around $\bar{u} \in \Omega$, we have

$$
N(\bar{u} ; \Theta)=\operatorname{Limsup}_{u \rightarrow{ }^{\rightarrow} \bar{u}} \widehat{N}(u ; \Theta) .
$$

The regular and Mordukhovich coderivatives of the multifunction $F: X \rightrightarrows W$ at the point $(\bar{u}, \bar{v}) \in \operatorname{gph} F$ are respectively the multifunction $\widehat{D}^{*} F(\bar{u}, \bar{v}): W^{*} \rightrightarrows X^{*}$ defined by

$$
\widehat{D}^{*} F(\bar{u}, \bar{v})\left(v^{*}\right)=\left\{u^{*} \in X^{*} \mid\left(u^{*},-v^{*}\right) \in \widehat{N}((\bar{u}, \bar{v}) ; \operatorname{gph} F)\right\}, \forall v^{*} \in W^{*},
$$

and the multifunction $D^{*} F(\bar{u}, \bar{v}): W^{*} \rightrightarrows X^{*}$ given by

$$
D^{*} F(\bar{u}, \bar{v})\left(v^{*}\right)=\left\{u^{*} \in X^{*} \mid\left(u^{*},-v^{*}\right) \in N((\bar{x}, \bar{v}) ; \operatorname{gph} F)\right\}, \forall v^{*} \in W^{*} .
$$


The multifunction $F$ is said to be normally regular at $(\bar{u}, \bar{v})$ if

$$
\widehat{D}^{*} F(\bar{u}, \bar{v})\left(v^{*}\right)=D^{*} F(\bar{u}, \bar{v})\left(v^{*}\right), \forall v^{*} \in W^{*} .
$$

If $F=f: X \rightarrow W$ is a single-valued function, we will write $\widehat{D}^{*} f(\bar{u})\left(v^{*}\right)$ and $D^{*} f(\bar{u})\left(v^{*}\right)$ for coderivatives of $f$ in (2.16) and (2.17). If $f$ is respectively Fréchet differentiable and strictly differentiable at $\bar{u}$, both the regular and Mordukhovich coderivatives are extensions of the corresponding adjoint derivative operators in the sense that

$$
\widehat{D}^{*} f(\bar{u})\left(v^{*}\right)=f^{\prime}(\bar{u})^{*} v^{*} \quad \text { and } \quad D^{*} f(\bar{u})\left(v^{*}\right)=f^{\prime}(\bar{u})^{*} v^{*}, \forall v^{*} \in W^{*} .
$$

The multifunction $F: X \rightrightarrows W$ is locally Lipschitz-like (or, $F$ has the Aubin property [12]) around a point $(\bar{u}, \bar{v}) \in \operatorname{gph} F$ if there exist $\ell>0$ and neighborhoods $U$ of $\bar{u}, V$ of $\bar{v}$ such that

$$
F\left(u_{1}\right) \cap V \subset F\left(u_{2}\right)+\ell\left\|u_{1}-u_{2}\right\| \bar{B}_{W}, \forall u_{1}, u_{2} \in U,
$$

where $\bar{B}_{W}$ denotes the closed unit ball in $W$. Characterization of this property via the mixed Mordukhovich coderivative of $F$ can be found in [18, Theorem 4.10]. Following Robinson [24], a single-valued function $h: D \subset X \rightarrow W$ is locally upper Lipschitzian at $\bar{u}$ if there exist $\eta>0$ and $\ell>0$ such that

$$
\|h(u)-h(\bar{u})\| \leq \ell\|u-\bar{u}\| \quad \text { whenever } \quad u \in B_{\eta}(\bar{u}) \cap D .
$$

We say that a multifunction $F: D \rightrightarrows W$ defined on some set $D \subset X$ admits a local upper Lipschitzian selection at $(\bar{u}, \bar{v}) \in \operatorname{gph} F$ if there is a single-valued function $h: D \rightarrow W$, which is locally upper Lipschitzian at $\bar{u}$ satisfying $h(\bar{u})=\bar{v}$ and $h(u) \in F(u)$ for all $u \in D$ in a neighborhood of $\bar{u}$. We also call $h$ a local upper Hölderian selection at $(\bar{u}, \bar{v}) \in \operatorname{gph} F$ if (2.18) is replaced by the Hölder property with some exponent $æ \geq 0$ below

$$
\|h(u)-h(\bar{u})\| \leq \ell\|u-\bar{u}\|^{\infty} \quad \text { whenever } \quad u \in B_{\eta}(\bar{u}) \cap D .
$$

\subsection{Assumptions and auxiliary results}

Let us assume that $\Omega \subset \mathbb{R}^{N}$ with $N \in\{1,2,3\}, \alpha, \beta \in L^{\infty}(\Omega), \alpha \leq \beta$, and $\alpha \not \equiv \beta$. Moreover, $L, f: \Omega \times \mathbb{R} \rightarrow \mathbb{R}$ are Carathéodory functions of class $\mathcal{C}^{2}$ with respect to the second variable satisfying the following assumptions.

(A1) The function $f(\cdot, 0) \in L^{\bar{p}}(\Omega)$ with $\bar{p}>N / 2$,

$$
\frac{\partial f}{\partial y}(x, y) \geq 0 \text { for a.a. } x \in \Omega,
$$

and for all $M>0$ there exists a constant $C_{f, M}>0$ such that

$$
\left|\frac{\partial f}{\partial y}(x, y)\right|+\left|\frac{\partial^{2} f}{\partial y^{2}}(x, y)\right| \leq C_{f, M} \quad \text { for a.a. } x \in \Omega \text { and }|y| \leq M .
$$

For every $M>0$ and $\varepsilon>0$ there exists $\delta>0$, depending on $M$ and $\varepsilon$ such that

$$
\left|\frac{\partial^{2} f}{\partial y^{2}}\left(x, y_{2}\right)-\frac{\partial^{2} f}{\partial y^{2}}\left(x, y_{1}\right)\right|<\varepsilon \quad \text { if }\left|y_{1}\right|,\left|y_{2}\right| \leq M,\left|y_{2}-y_{1}\right| \leq \delta \text {, and for a.a. } x \in \Omega \text {. }
$$

(A2) The function $L(\cdot, 0) \in L^{1}(\Omega)$ and for all $M>0$ there are a constant $C_{L, M}>0$ and a function $\psi_{M} \in L^{\bar{p}}(\Omega)$ such that for every $|y| \leq M$ and almost all $x \in \Omega$,

$$
\left|\frac{\partial L}{\partial y}(x, y)\right| \leq \psi_{M}(x), \quad\left|\frac{\partial^{2} L}{\partial y^{2}}(x, y)\right| \leq C_{L, M}
$$


For every $M>0$ and $\varepsilon>0$ there exists $\delta>0$, depending on $M$ and $\varepsilon$ such that

$$
\left|\frac{\partial^{2} L}{\partial y^{2}}\left(x, y_{2}\right)-\frac{\partial^{2} L}{\partial y^{2}}\left(x, y_{1}\right)\right|<\varepsilon \quad \text { if }\left|y_{1}\right|,\left|y_{2}\right| \leq M,\left|y_{2}-y_{1}\right| \leq \delta \text {, and for a.a. } x \in \Omega \text {. }
$$

(A3) The set $\Omega$ is an open and bounded domain in $\mathbb{R}^{N}$ with Lipschitz boundary $\Gamma$. The set $\mathcal{Q}$ is closed, convex, and bounded in $L^{p_{0}}(\Omega)$ satisfying $\mathcal{U}_{a d}(e) \cap \operatorname{int} \mathcal{Q} \neq \emptyset$ for some $e \in E$, where int $\mathcal{Q}$ stands for the interior of $\mathcal{Q}$. The coefficients $a_{i j} \in C(\bar{\Omega})$ of the second-order elliptic differential operator $A$ satisfy

$$
\lambda_{A}\|\xi\|_{\mathbb{R}^{N}}^{2} \leq \sum_{i, j=1}^{N} a_{i j}(x) \xi_{i} \xi_{j}, \forall \xi \in \mathbb{R}^{N}, \text { for a.a. } x \in \Omega
$$

for some constant $\lambda_{A}>0$.

For every $u \in L^{p}(\Omega)$ with $p>N / 2$, according to [31, Chapter 4], equation (2.2) has a unique weak solution $y_{u} \in H_{0}^{1}(\Omega) \cap C(\bar{\Omega})$. In addition, there exists a constant $M_{\alpha, \beta}>0$ such that

$$
\left\|y_{u}\right\|_{H_{0}^{1}(\Omega)}+\left\|y_{u}\right\|_{C(\bar{\Omega})} \leq M_{\alpha, \beta}, \forall u \in \mathcal{U}_{a d} .
$$

The control-to-state mapping $G: L^{p}(\Omega) \rightarrow H_{0}^{1}(\Omega) \cap C(\bar{\Omega})$ defined by $G(u)=y_{u}$ is of class $\mathcal{C}^{2}$. Moreover, for every $v \in L^{2}(\Omega), z_{u, v}=G^{\prime}(u) v$ is the unique weak solution of

$$
\left\{\begin{aligned}
A z+\frac{\partial f}{\partial y}(x, y) z & =v & & \text { in } \Omega \\
z & =0 & & \text { on } \Gamma,
\end{aligned}\right.
$$

and for any $v_{1}, v_{2} \in L^{2}(\Omega), w_{v_{1}, v_{2}}=G^{\prime \prime}(u)\left(v_{1}, v_{2}\right)$ is the unique weak solution of

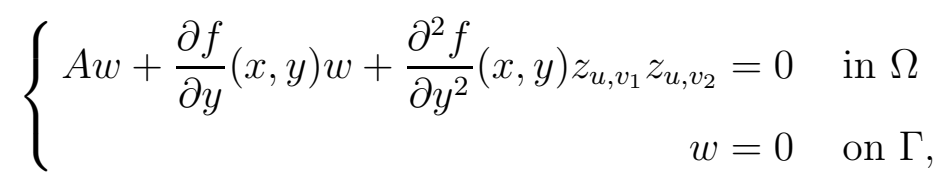

where $y=G(u)$ and $z_{u, v_{i}}=G^{\prime}(u) v_{i}$ for $i=1,2$.

By assumption (A2), using the latter results and applying the chain rule we deduce that the cost functional $J: L^{p}(\Omega) \rightarrow \mathbb{R}$ with $p>N / 2$ is of class $\mathcal{C}^{2}$, and the first and second derivatives of $J(\cdot)$ are given by

$$
J^{\prime}(u) v=\int_{\Omega}\left(\varphi_{u}+\zeta u\right) v d x
$$

and

$$
J^{\prime \prime}(u)\left(v_{1}, v_{2}\right)=\int_{\Omega}\left(\frac{\partial^{2} L}{\partial y^{2}}\left(x, y_{u}\right) z_{u, v_{1}} z_{u, v_{2}}+\zeta v_{1} v_{2}-\varphi_{u} \frac{\partial^{2} f}{\partial y^{2}}\left(x, y_{u}\right) z_{u, v_{1}} z_{u, v_{2}}\right) d x
$$

where $z_{u, v_{i}}=G^{\prime}(u) v_{i}$ for $i=1,2$, and $\varphi_{u} \in H_{0}^{1}(\Omega) \cap C(\bar{\Omega})$ is the adjoint state of $y_{u}$ defined as the unique weak solution of

$$
\left\{\begin{aligned}
A^{*} \varphi+\frac{\partial f}{\partial y}\left(x, y_{u}\right) \varphi & =\frac{\partial L}{\partial y}\left(x, y_{u}\right) & & \text { in } \Omega \\
\varphi & =0 & & \text { on } \Gamma
\end{aligned}\right.
$$

where $A^{*}$ is the adjoint operator of $A$. 
A control $\bar{u} \in \mathcal{U}_{a d}$ is said to be a solution/global minimum of problem (2.1) if $J(\bar{u}) \leq J(u)$ for all $u \in \mathcal{U}_{a d}$. We will say that $\bar{u}$ is a local solution/local minimum of problem (2.1) in the sense of $L^{p}(\Omega)$ if there exists a closed ball $\bar{B}_{\varepsilon}^{p}(\bar{u})$ such that $J(\bar{u}) \leq J(u)$ for all $u \in \mathcal{U}_{a d} \cap \bar{B}_{\varepsilon}^{p}(\bar{u})$. The local solution $\bar{u}$ is called strict if $J(\bar{u})<J(u)$ holds for all $u \in \mathcal{U}_{a d} \cap \bar{B}_{\varepsilon}^{p}(\bar{u})$ with $u \neq \bar{u}$. Under the assumptions given above, solutions of problem (2.1) exist. We introduce the space $Y=H_{0}^{1}(\Omega) \cap C(\bar{\Omega})$ endowed with the norm

$$
\|y\|_{Y}=\|y\|_{H_{0}^{1}(\Omega)}+\|y\|_{L^{\infty}(\Omega)} .
$$

According to [31, Chapter 4], if $\bar{u} \in \mathcal{U}_{a d}$ is a solution of problem (2.1) in the sense of $L^{p}(\Omega)$, then there exist a unique state $y_{\bar{u}} \in Y$ and a unique adjoint state $\varphi_{\bar{u}} \in Y$ satisfying the first-order optimality system

$$
\begin{gathered}
\left\{\begin{aligned}
A y_{\bar{u}}+f\left(x, y_{\bar{u}}\right) & =\bar{u} \quad \text { in } \Omega \\
y_{\bar{u}}=0 \quad & \text { on } \Gamma,
\end{aligned}\right. \\
\left\{\begin{aligned}
A^{*} \varphi_{\bar{u}}+\frac{\partial f}{\partial y}\left(x, y_{\bar{u}}\right) \varphi_{\bar{u}}=\frac{\partial L}{\partial y}\left(x, y_{\bar{u}}\right) & \text { in } \Omega \\
\varphi_{\bar{u}}=0 & \text { on } \Gamma,
\end{aligned}\right. \\
\int_{\Omega}\left(\varphi_{\bar{u}}+\zeta \bar{u}\right)(u-\bar{u}) d x \geq 0, \forall u \in \mathcal{U}_{a d} .
\end{gathered}
$$

Similarly, if $\bar{u}_{e} \in \mathcal{G}(e)$ is a solution of the perturbed problem (2.3) with respect to $e \in E$, then $\bar{u}_{e}$ satisfies the perturbed first-order optimality system

$$
\begin{gathered}
\left\{\begin{aligned}
A y_{\bar{u}_{e}+e_{y}}+f\left(x, y_{\bar{u}_{e}+e_{y}}\right)=\bar{u}_{e}+e_{y} & \text { in } \Omega \\
y_{\bar{u}_{e}+e_{y}}=0 & \text { on } \Gamma,
\end{aligned}\right. \\
\left\{\begin{array}{cl}
A^{*} \varphi_{\bar{u}_{e}, e}+\frac{\partial f}{\partial y}\left(x, y_{\bar{u}_{e}+e_{y}}\right) \varphi_{\bar{u}_{e}, e}=\frac{\partial L}{\partial y}\left(x, y_{\bar{u}_{e}+e_{y}}\right)+e_{J} & \text { in } \Omega \\
\varphi_{\bar{u}_{e}, e}=0 & \text { on } \Gamma,
\end{array}\right. \\
\int_{\Omega}\left(\varphi_{\bar{u}_{e}, e}+\zeta \bar{u}_{e}\right)\left(u(x)-\bar{u}_{e}(x)\right) d x \geq 0, \forall u \in \mathcal{G}(e),
\end{gathered}
$$

where $\varphi_{\bar{u}_{e}, e}$ is the adjoint state of $y_{\bar{u}_{e}+e_{y}}$ for the perturbed problem (2.3). Furthermore, the partial derivative of $\mathcal{J}(u, e)$ in $u$ at $\bar{u}_{e}$ can be computed by

$$
\mathcal{J}_{u}^{\prime}\left(\bar{u}_{e}, e\right) v=\int_{\Omega}\left(\varphi_{\bar{u}_{e}, e}+\zeta \bar{u}_{e}\right) v d x
$$

\section{Subgradients of marginal functions}

In this section, we consider the parametric control problem (2.3), where $p_{0}=2$ while $q_{0}=2$ in (2.5), $p_{1}=p_{2}=p_{3}=p_{4}=2$ in (2.6) , and $s_{0}=2$. This means that $\mathcal{Q} \subset L^{2}(\Omega)$ and the perturbed admissible control set $\mathcal{U}_{a d}(e) \subset L^{2}(\Omega)$ for $e \in E=L^{2}(\Omega) \times L^{2}(\Omega) \times L^{2}(\Omega) \times L^{2}(\Omega)$.

Theorem 3.1. Assume that the assumptions (A1)-(A3) hold. Then, for each $e \in E$ with $\mathcal{G}(e) \neq \emptyset$, the perturbed control problem (2.3) has at least one optimal control $\bar{u}_{e}$ with associated optimal perturbed state $y_{\bar{u}_{e}+e_{y}} \in H^{1}(\Omega) \cap C(\bar{\Omega})$.

Proof. Let $e \in E$ be such that $\mathcal{G}(e) \neq \emptyset$. Then, $\mathcal{G}(e)$ is nonempty, closed, bounded, and convex in $L^{2}(\Omega)$ due to $\mathcal{U}_{a d}(e)$ is closed, bounded, and convex in $L^{2}(\Omega)$. By arguing similarly as in the proof of [23, Theorem 4.1], we obtain assertion of the theorem. 


\subsection{Regular subgradients of marginal functions}

Let the marginal function $\mu(\cdot)$ from (2.7) be finite at some $\bar{e} \in \operatorname{dom} S$, and let $\bar{u}_{\bar{e}} \in S(\bar{e})$ be such that $\widehat{\partial}^{+} \mathcal{J}\left(\bar{u}_{\bar{e}}, \bar{e}\right) \neq \emptyset$. Then, applying [21, Theorem 1], we obtain

$$
\widehat{\partial} \mu(\bar{e}) \subset \bigcap_{\left(u^{*}, e^{*}\right) \in \widehat{\partial}^{+} \mathcal{J}\left(\bar{u}_{\bar{e}}, \bar{e}\right)}\left(e^{*}+\widehat{D}^{*} \mathcal{G}\left(\bar{e}, \bar{u}_{\bar{e}}\right)\left(u^{*}\right)\right) .
$$

Note that by the assumptions (A1)-(A3) the function $\mathcal{J}$ is Fréchet differentiable at $\left(\bar{u}_{\bar{e}}, \bar{e}\right)$. Thus, we get

$$
\widehat{\partial}^{+} \mathcal{J}\left(\bar{u}_{\bar{e}}, \bar{e}\right)=\left\{\nabla \mathcal{J}\left(\bar{u}_{\bar{e}}, \bar{e}\right)\right\}=\left\{\left(\mathcal{J}_{u}^{\prime}\left(\bar{u}_{\bar{e}}, \bar{e}\right), \mathcal{J}_{e}^{\prime}\left(\bar{u}_{\bar{e}}, \bar{e}\right)\right)\right\} .
$$

Consequently, from (3.1) we deduce that

$$
\widehat{\partial} \mu(\bar{e}) \subset \mathcal{J}_{e}^{\prime}\left(\bar{u}_{\bar{e}}, \bar{e}\right)+\widehat{D}^{*} \mathcal{G}\left(\bar{e}, \bar{u}_{\bar{e}}\right)\left(\mathcal{J}_{u}^{\prime}\left(\bar{u}_{\bar{e}}, \bar{e}\right)\right) .
$$

If, in addition, the solution map $S: \operatorname{dom} \mathcal{G} \rightrightarrows L^{2}(\Omega)$ admits a local upper Lipschitzian selection at $\left(\bar{e}, \bar{u}_{\bar{e}}\right)$, then by [21, Theorem 2] we obtain

$$
\widehat{\partial} \mu(\bar{e})=\mathcal{J}_{e}^{\prime}\left(\bar{u}_{\bar{e}}, \bar{e}\right)+\widehat{D}^{*} \mathcal{G}\left(\bar{e}, \bar{u}_{\bar{e}}\right)\left(\mathcal{J}_{u}^{\prime}\left(\bar{u}_{\bar{e}}, \bar{e}\right)\right) .
$$

We will apply (3.2) to derive a new explicit formula for estimating the Fréchet subdifferential $\widehat{\partial} \mu(\bar{e})$, and this formula will also be an exact formula for computing $\widehat{\partial} \mu(\bar{e})$ provided that the solution map $S(\cdot)$ has a local upper Lipschitzian selection at $\left(\bar{e}, \bar{u}_{\bar{e}}\right)$.

For each pair $(e, u) \in E \times L^{2}(\Omega)$ with $u \in \mathcal{G}(e)$ we define subsets $\Omega_{1}(e, u), \Omega_{2}(e, u)$, $\Omega_{3}(e, u)$ of $\Omega$ by

$$
\left\{\begin{array}{l}
\Omega_{1}(e, u)=\left\{x \in \Omega \mid u(x)=\alpha(x)+e_{\alpha}(x)\right\} \\
\Omega_{2}(e, u)=\left\{x \in \Omega \mid u(x) \in\left(\alpha(x)+e_{\alpha}(x), \beta(x)+e_{\beta}(x)\right)\right\} \\
\Omega_{3}(e, u)=\left\{x \in \Omega \mid u(x)=\beta(x)+e_{\beta}(x)\right\}
\end{array}\right.
$$

We have gph $\mathcal{G}=\operatorname{gph} \mathcal{U}_{a d} \cap(E \times \mathcal{Q})$, where gph $\mathcal{U}_{a d}$ and $E \times \mathcal{Q}$ are convex sets. In addition, we can verify that $\operatorname{gph} \mathcal{U}_{a d} \cap \operatorname{int}(E \times \mathcal{Q}) \neq \emptyset$. By [17, Proposition 1, p. 205], we get

$$
\begin{aligned}
\widehat{N}((e, u) ; \operatorname{gph} \mathcal{G}) & =\widehat{N}\left((e, u) ; \operatorname{gph} \mathcal{U}_{a d}\right)+\widehat{N}((e, u) ; E \times \mathcal{Q}) \\
& =\widehat{N}\left((e, u) ; \operatorname{gph} \mathcal{U}_{a d}\right)+\left\{0_{E}\right\} \times N(u ; \mathcal{Q})
\end{aligned}
$$

Thus, for each $\left(\bar{e}, \bar{u}_{\bar{e}}\right) \in \operatorname{gph} S$, we obtain

$$
\begin{aligned}
\widehat{D}^{*} \mathcal{G}\left(\bar{e}, \bar{u}_{\bar{e}}\right)\left(u^{*}\right) & =\left\{e^{*} \in E^{*} \mid\left(e^{*},-u^{*}\right) \in \widehat{N}\left(\left(\bar{e}, \bar{u}_{\bar{e}}\right) ; \operatorname{gph} \mathcal{G}\right)\right\} \\
& =\left\{e^{*} \in E^{*} \mid\left(e^{*},-u^{*}\right) \in \widehat{N}\left(\left(\bar{e}, \bar{u}_{\bar{e}}\right) ; \operatorname{gph} \mathcal{U}_{a d}\right)+\left\{0_{E}\right\} \times N\left(\bar{u}_{\bar{e}} ; \mathcal{Q}\right)\right\}
\end{aligned}
$$

In order to compute $\widehat{D}^{*} \mathcal{G}\left(\bar{e}, \bar{u}_{\bar{e}}\right)\left(u^{*}\right)$ explicitly via (3.5) , we provide a formula for computing the regular normal cone $\widehat{N}\left(\left(\bar{e}, \bar{u}_{\bar{e}}\right) ;\right.$ gph $\left.\mathcal{U}_{a d}\right)$ in the following lemma.

Lemma 3.2. Assume that the assumptions (A1)-(A3) hold and let $\bar{u}_{\bar{e}} \in S(\bar{e})$. The following formula holds that

$$
\begin{aligned}
\widehat{N}\left(\left(\bar{e}, \bar{u}_{\bar{e}}\right) ; \operatorname{gph} \mathcal{U}_{a d}\right)=\{ & \left(e^{*}, u^{*}\right) \in E^{*} \times L^{2}(\Omega) \mid e^{*}=\left(0,0, e_{\alpha}^{*}, e_{\beta}^{*}\right), u^{*}=-e_{\alpha}^{*}-e_{\beta}^{*}, \\
& \left.e_{\alpha}^{*}\right|_{\Omega_{1}\left(\bar{e}, \bar{u}_{\bar{e}}\right)} \geq 0,\left.e_{\alpha}^{*}\right|_{\Omega \backslash \Omega_{1}\left(\bar{e}, \bar{u}_{\bar{e}}\right)}=0 \\
& \left.\left.e_{\beta}^{*}\right|_{\Omega_{3}\left(\bar{e}, \bar{u}_{\bar{e}}\right)} \leq 0,\left.e_{\beta}^{*}\right|_{\Omega \backslash \Omega_{3}\left(\bar{e}, \bar{u}_{\bar{e}}\right)}=0\right\}
\end{aligned}
$$


Proof. Let $\left(e^{*}, u^{*}\right) \in \widehat{N}\left(\left(\bar{e}, \bar{u}_{\bar{e}}\right) ; \operatorname{gph} \mathcal{U}_{a d}\right)$ with $e^{*}=\left(e_{y}^{*}, e_{J}^{*}, e_{\alpha}^{*}, e_{\beta}^{*}\right) \in E^{*}$. Since the set $\mathcal{U}_{a d}(e)$ does not depend on $e_{y}$ and $e_{J}$ for every $e=\left(e_{y}, e_{J}, e_{\alpha}, e_{\beta}\right) \in E$, we have $e_{y}^{*}=e_{J}^{*}=0$. By the definition of $\widehat{N}\left(\left(\bar{e}, \bar{u}_{\bar{e}}\right)\right.$; gph $\left.\mathcal{U}_{a d}\right)$, we have

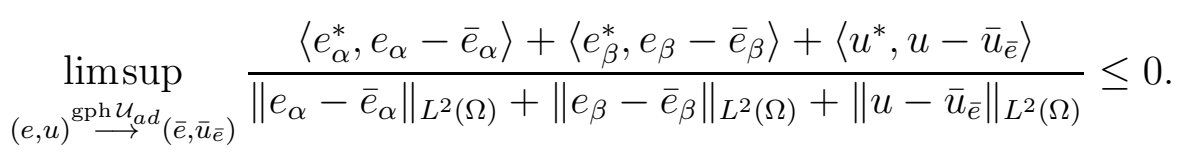

Let $e_{\alpha}-\bar{e}_{\alpha}=e_{\beta}-\bar{e}_{\beta}=u-\bar{u}_{\bar{e}}$. Then, $(e, u) \in \operatorname{gph} \mathcal{U}_{a d}$ and from (3.7) we have

$$
\limsup _{e_{\alpha} \rightarrow \bar{e}_{\alpha}} \frac{\left\langle e_{\alpha}^{*}+e_{\beta}^{*}+u^{*}, e_{\alpha}-\bar{e}_{\alpha}\right\rangle}{\left\|e_{\alpha}-\bar{e}_{\alpha}\right\|_{L^{2}(\Omega)}} \leq 0 .
$$

This yields $e_{\alpha}^{*}+e_{\beta}^{*}+u^{*}=0$, or $u^{*}=-e_{\alpha}^{*}-e_{\beta}^{*}$. We now consider the following situations.

- Necessary conditions for $e_{\alpha}^{*}$ :

. Let $e_{\beta}=\bar{e}_{\beta}, u=\bar{u}_{\bar{e}},\left.\left.e_{\alpha}\right|_{\Omega_{1}\left(\bar{e}, \bar{u}_{\bar{e}}\right)} \nearrow \bar{e}_{\alpha}\right|_{\Omega_{1}\left(\bar{e}, \bar{u}_{\bar{e}}\right)},\left.e_{\alpha}\right|_{\Omega \backslash \Omega_{1}\left(\bar{e}, \bar{u}_{\bar{e}}\right)}=\left.\bar{e}_{\alpha}\right|_{\Omega \backslash \Omega_{1}\left(\bar{e}, \bar{u}_{\bar{e}}\right)}$. Then, we have $(e, u) \in \operatorname{gph} \mathcal{U}_{a d}$ and from (3.7) we deduce that $\left.e_{\alpha}^{*}\right|_{\Omega_{1}\left(\bar{e}, \bar{u}_{\bar{e}}\right)} \geq 0$.

- Let $e_{\beta}=\bar{e}_{\beta}$ and $u=\bar{u}_{\bar{e}}$. For $\varepsilon>0$, define $A_{\varepsilon}:=\left\{x \in \Omega \mid \bar{u}_{\bar{e}} \geq \alpha+\bar{e}_{\alpha}+\varepsilon\right\} \subset \Omega \backslash \Omega_{1}(\bar{e}, \bar{u})$. Let now $B \subset A_{\varepsilon}$ with positive measure. Let us set $\left.e_{\alpha}\right|_{\Omega_{1}\left(\bar{e}, \bar{u}_{\bar{e}}\right)}=\left.\bar{e}_{\alpha}\right|_{\Omega_{1}\left(\bar{e}, \bar{u}_{\bar{e}}\right)}$ and

$$
\left.e_{\alpha}\right|_{\Omega \backslash \Omega_{1}\left(\bar{e}, \bar{u}_{\bar{e}}\right)}=\left.\bar{e}_{\alpha}\right|_{\Omega \backslash \Omega_{1}\left(\bar{e}, \bar{u}_{\bar{e}}\right)}+t \chi_{B},
$$

where $|t|<\varepsilon$. Then, we have $(e, u) \in \operatorname{gph} \mathcal{U}_{a d}$, and from (3.7) we deduce

$$
0 \geq \limsup _{e_{\alpha} \rightarrow \bar{e}_{\alpha}} \frac{\left\langle e_{\alpha}^{*}, e_{\alpha}-\bar{e}_{\alpha}\right\rangle}{\left\|e_{\alpha}-\bar{e}_{\alpha}\right\|_{L^{2}(\Omega)}} \geq \limsup _{t \rightarrow 0} \frac{\left\langle e_{\alpha}^{*}, t \chi_{B}\right\rangle}{\left\|t \chi_{B}\right\|_{L^{2}(\Omega)}}=\left\|\chi_{B}\right\|_{L^{2}(\Omega)}^{-1}\left|\left\langle e_{\alpha}^{*}, \chi_{B}\right\rangle\right| .
$$

This implies $e_{\alpha}^{*}=0$ almost everywhere on $A_{\varepsilon}$. Since $\cup_{\varepsilon>0} A_{\varepsilon}=\Omega \backslash \Omega_{1}\left(\bar{e}, \bar{u}_{\bar{e}}\right)$, we find $e_{\alpha}^{*}=0$ almost everywhere on $\Omega \backslash \Omega_{1}\left(\bar{e}, \bar{u}_{\bar{e}}\right)$ as claimed.

- Necessary conditions for $e_{\beta}^{*}$ :

- Let $e_{\alpha}=\bar{e}_{\alpha}, u=\bar{u}_{\bar{e}},\left.e_{\beta}\right|_{\Omega \backslash \Omega_{3}\left(\bar{e}, \bar{u}_{\bar{e}}\right)}=\left.\bar{e}_{\beta}\right|_{\Omega \backslash \Omega_{3}\left(\bar{e}, \bar{u}_{\bar{e}}\right)},\left.\left.e_{\beta}\right|_{\Omega_{3}\left(\bar{e}, \bar{u}_{\bar{e}}\right)} \searrow \bar{e}_{\beta}\right|_{\Omega_{3}\left(\bar{e}, \bar{u}_{\bar{e}}\right)}$. Then, we have $(e, u) \in \operatorname{gph} \mathcal{U}_{a d}$ and from (3.7) we deduce that $\left.e_{\beta}^{*}\right|_{\Omega_{3}\left(\bar{e}, \bar{u}_{\bar{e}}\right)} \leq 0$.

- By arguing similarly as in the proof of the second necessary condition for $e_{\alpha}^{*}$ above, we also find that $\left.e_{\beta}^{*}\right|_{\Omega \backslash \Omega_{3}\left(\bar{e}, \bar{u}_{\bar{e}}\right)}=0$.

Conversely, pick any $\left(e^{*}, u^{*}\right)$ from the set on the right-hand side of (3.6). Taking any sequence $\left(e_{n}, u_{n}\right) \rightarrow\left(\bar{e}, \bar{u}_{\bar{e}}\right)$ with $\left(e_{n}, u_{n}\right) \in \operatorname{gph} \mathcal{U}_{a d}$, we have to show that (3.7) holds for this sequence. For convenience, we denote $\Omega_{i}=\Omega_{i}\left(\bar{e}, \bar{u}_{\bar{e}}\right)$ for $i=1,2,3$. We observe that

$$
\begin{aligned}
& \left\langle e_{\alpha}^{*},\left(e_{\alpha}\right)_{n}-\bar{e}_{\alpha}\right\rangle+\left\langle e_{\beta}^{*},\left(e_{\beta}\right)_{n}-\bar{e}_{\beta}\right\rangle+\left\langle u^{*}, u_{n}-\bar{u}_{\bar{e}}\right\rangle \\
= & \left\langle\left. e_{\alpha}^{*}\right|_{\Omega_{1}},\left.\left(e_{\alpha}\right)_{n}\right|_{\Omega_{1}}-\left.\bar{e}_{\alpha}\right|_{\Omega_{1}}\right\rangle+\left\langle\left. e_{\beta}^{*}\right|_{\Omega_{3}},\left.\left(e_{\beta}\right)_{n}\right|_{\Omega_{3}}-\left.\bar{e}_{\beta}\right|_{\Omega_{3}}\right\rangle \\
& -\left\langle\left.\left(e_{\alpha}^{*}+e_{\beta}^{*}\right)\right|_{\Omega_{1} \cup \Omega_{3}},\left.u_{n}\right|_{\Omega_{1} \cup \Omega_{3}}-\left.\bar{u}_{\bar{e}}\right|_{\Omega_{1} \cup \Omega_{3}}\right\rangle \\
= & \left\langle\left. e_{\alpha}^{*}\right|_{\Omega_{1}},\left.\left(e_{\alpha}\right)_{n}\right|_{\Omega_{1}}-\left.\bar{e}_{\alpha}\right|_{\Omega_{1}}\right\rangle+\left\langle\left. e_{\beta}^{*}\right|_{\Omega_{3}},\left.\left(e_{\beta}\right)_{n}\right|_{\Omega_{3}}-\left.\bar{e}_{\beta}\right|_{\Omega_{3}}\right\rangle \\
& -\left\langle e_{\alpha}^{*}\left|\Omega_{\Omega_{1}}, u_{n}\right|_{\Omega_{1}}-\left.\bar{u}_{\bar{e}}\right|_{\Omega_{1}}\right\rangle-\left\langle\left. e_{\beta}^{*}\right|_{\Omega_{3}},\left.u_{n}\right|_{\Omega_{3}}-\left.\bar{u}_{\bar{e}}\right|_{\Omega_{3}}\right\rangle \\
= & \left\langle\left. e_{\alpha}^{*}\right|_{\Omega_{1}},\left.\left(e_{\alpha}\right)_{n}\right|_{\Omega_{1}}-\left.\bar{e}_{\alpha}\right|_{\Omega_{1}}-\left.u_{n}\right|_{\Omega_{1}}+\left.\bar{u}_{\bar{e}}\right|_{\Omega_{1}}\right\rangle+\left\langle\left. e_{\beta}^{*}\right|_{\Omega_{3}},\left.\left(e_{\beta}\right)_{n}\right|_{\Omega_{3}}-\left.\bar{e}_{\beta}\right|_{\Omega_{3}}-\left.u_{n}\right|_{\Omega_{3}}+\left.\bar{u}_{\bar{e}}\right|_{\Omega_{3}}\right\rangle \\
& \left(\text { with }\left.\bar{u}_{\bar{e}}\right|_{\Omega_{1}}=\left.\alpha\right|_{\Omega_{1}}+\left.\bar{e}_{\alpha}\right|_{\Omega_{1}} \text { and }\left.\bar{u}_{\bar{e}}\right|_{\Omega_{3}}=\left.\beta\right|_{\Omega_{3}}+\left.\bar{e}_{\beta}\right|_{\Omega_{3}} \text { by the definition of } \Omega_{1} \text { and } \Omega_{3}\right) \\
= & \langle\underbrace{\left.e_{\alpha}^{*}\right|_{\Omega_{1}}}_{\geq 0}, \underbrace{\left.\left.\alpha\right|_{\Omega_{1}}+\left.\left(e_{\alpha}\right)_{n}\right|_{\Omega_{1}}-\left.u_{n}\right|_{\Omega_{1}}\right\rangle}_{\leq 0}+\langle\underbrace{\left.e_{\beta}^{*}\right|_{\Omega_{3}}}_{\leq 0}, \underbrace{\beta 0}_{\left.\Omega_{\Omega_{3}}+\left.\left(e_{\beta}\right)_{n}\right|_{\Omega_{3}}-\left.u_{n}\right|_{\Omega_{3}}\right\rangle} \\
\leq & 0 .
\end{aligned}
$$

This implies that (3.7) holds for the sequence $\left\{\left(e_{n}, u_{n}\right)\right\}$ chosen above. 
Proposition 3.3. Assume that the assumptions (A1)-(A3) hold and let $\bar{u}_{\bar{e}} \in S(\bar{e})$. Then, the following formula holds that

$$
\begin{aligned}
& \widehat{D}^{*} \mathcal{G}\left(\bar{e}, \bar{u}_{\bar{e}}\right)\left(u^{*}\right)=\left\{e^{*} \in E^{*} \mid e^{*}=\left(0,0, e_{\alpha}^{*}, e_{\beta}^{*}\right), u^{*}=u_{1}^{*}-u_{2}^{*},\right. \\
& u_{1}^{*}=e_{\alpha}^{*}+e_{\beta}^{*}, u_{2}^{*} \in N\left(\bar{u}_{\bar{e}} ; \mathcal{Q}\right) \\
&\left.e_{\alpha}^{*}\right|_{\Omega_{1}\left(\bar{e}, \bar{u}_{\bar{e}}\right)} \geq 0,\left.e_{\alpha}^{*}\right|_{\Omega \backslash \Omega_{1}\left(\bar{e}, \bar{u}_{\bar{e}}\right)}=0 \\
&\left.\left.e_{\beta}^{*}\right|_{\Omega_{3}\left(\bar{e}, \bar{u}_{\bar{e}}\right)} \leq 0,\left.e_{\beta}^{*}\right|_{\Omega \backslash \Omega_{3}\left(\bar{e}, \bar{u}_{\bar{e}}\right)}=0\right\}
\end{aligned}
$$

Proof. Formula (3.8) follows directly from (3.5) and (3.6).

The forthcoming theorem establishes an upper estimate for the regular subdifferential of the marginal function $\mu(\cdot)$.

Theorem 3.4. Assume that the assumptions (A1)-(A3) hold and let $\bar{u}_{\bar{e}} \in S(\bar{e})$. Then it is necessary for an element $\widehat{e}^{*}=\left(\widehat{e}_{y}^{*}, \widehat{e}_{J}^{*}, \widehat{e}_{\alpha}^{*}, \widehat{e}_{\beta}^{*}\right)$ from $E^{*}$ belonging to $\widehat{\partial} \mu(\bar{e})$ that

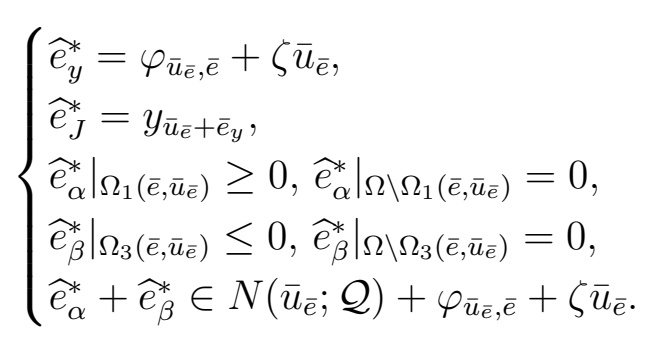

If, in addition, $S: \operatorname{dom} \mathcal{G} \rightrightarrows L^{2}(\Omega)$ has a local upper Lipschitzian selection at $\left(\bar{e}, \bar{u}_{\bar{e}}\right)$, then condition (3.9) is also sufficient for the inclusion $\widehat{e}^{*} \in \widehat{\partial} \mu(\bar{e})$.

Proof. Pick any $\widehat{e}^{*}=\left(\widehat{e}_{y}^{*}, \widehat{e}_{J}^{*}, \widehat{e}_{\alpha}^{*}, \widehat{e}_{\beta}^{*}\right) \in \widehat{\partial} \mu(\bar{e})$. Using (3.2) we obtain the following inclusion

$$
\widehat{e}^{*} \in \mathcal{J}_{e}^{\prime}\left(\bar{u}_{\bar{e}}, \bar{e}\right)+\widehat{D}^{*} \mathcal{G}\left(\bar{e}, \bar{u}_{\bar{e}}\right)\left(\mathcal{J}_{u}^{\prime}\left(\bar{u}_{\bar{e}}, \bar{e}\right)\right),
$$

or, equivalently, as follows

$$
\widehat{e}^{*}-\mathcal{J}_{e}^{\prime}\left(\bar{u}_{\bar{e}}, \bar{e}\right) \in \widehat{D}^{*} \mathcal{G}\left(\bar{e}, \bar{u}_{\bar{e}}\right)\left(\mathcal{J}_{u}^{\prime}\left(\bar{u}_{\bar{e}}, \bar{e}\right)\right) .
$$

From (2.3) we have

$$
\mathcal{J}(u, e)=J\left(u+e_{y}\right)+\left(e_{J}, G\left(u+e_{y}\right)\right)_{L^{2}(\Omega)} \cdot
$$

It follows that

$$
\mathcal{J}_{u}^{\prime}\left(\bar{u}_{\bar{e}}, \bar{e}\right) v=J^{\prime}\left(\bar{u}_{\bar{e}}+\bar{e}_{y}\right) v+\left(\bar{e}_{J}, G^{\prime}\left(\bar{u}_{\bar{e}}+\bar{e}_{y}\right) v\right)_{L^{2}(\Omega)},
$$

and

$$
\begin{aligned}
\mathcal{J}_{e}^{\prime}\left(\bar{u}_{\bar{e}}, \bar{e}\right) \widetilde{e} & =J^{\prime}\left(\bar{u}_{\bar{e}}+\bar{e}_{y}\right) \widetilde{e}_{y}+\left(\bar{e}_{J}, G^{\prime}\left(\bar{u}_{\bar{e}}+\bar{e}_{y}\right) \widetilde{e}_{y}\right)_{L^{2}(\Omega)}+\left(\widetilde{e}_{J}, G\left(\bar{u}_{\bar{e}}+\bar{e}_{y}\right)\right)_{L^{2}(\Omega)} \\
& =\mathcal{J}_{u}^{\prime}\left(\bar{u}_{\bar{e}}, \bar{e}\right) \widetilde{e}_{y}+\left(\widetilde{e}_{J}, y_{\bar{u}_{\bar{e}}+\bar{e}_{y}}\right)_{L^{2}(\Omega)}
\end{aligned}
$$

where $y_{\bar{u}_{\bar{e}}+\bar{e}_{y}}=G\left(\bar{u}_{\bar{e}}+\bar{e}_{y}\right)$. Using (2.32) we find that $\mathcal{J}_{u}^{\prime}\left(\bar{u}_{\bar{e}}, \bar{e}\right)=\varphi_{\bar{u}_{\bar{e}}, \bar{e}}+\zeta \bar{u}_{\bar{e}}$. Combining this with (3.11) and the fact that $\mathcal{J}(u, e)$ does not depend on $e_{\alpha}$ and $e_{\beta}$, we obtain

$$
\mathcal{J}_{e}^{\prime}\left(\bar{u}_{\bar{e}}, \bar{e}\right)=\left(\varphi_{\bar{u}_{\bar{e}}, \bar{e}}+\zeta \bar{u}_{\bar{e}}, y_{\bar{u}_{\bar{e}}+\bar{e}_{y}}, 0_{L^{2}(\Omega)}, 0_{L^{2}(\Omega)}\right) .
$$

Consequently, we have

$$
\widehat{e}^{*}-\mathcal{J}_{e}^{\prime}\left(\bar{u}_{\bar{e}}, \bar{e}\right)=\left(\widehat{e}_{y}^{*}-\varphi_{\bar{u}_{\bar{e}}, \bar{e}}-\zeta \bar{u}_{\bar{e}}, \widehat{e}_{J}^{*}-y_{\bar{u}_{\bar{e}}+\bar{e}_{y}}, \widehat{e}_{\alpha}^{*}, \widehat{e}_{\beta}^{*}\right) .
$$


From (3.12), (3.10) and (3.8) we deduce that

$$
\widehat{e}_{y}^{*}-\varphi_{\bar{u}_{\bar{e}}, \bar{e}}-\zeta \bar{u}_{\bar{e}}=0, \quad \widehat{e}_{J}^{*}-y_{\bar{u}_{\bar{e}}+\bar{e}_{y}}=0, \quad \widehat{e}_{\alpha}^{*}=e_{\alpha}^{*}, \quad \widehat{e}_{\beta}^{*}=e_{\beta}^{*},
$$

where $e_{\alpha}^{*}$ and $e_{\beta}^{*}$ satisfy the following condition

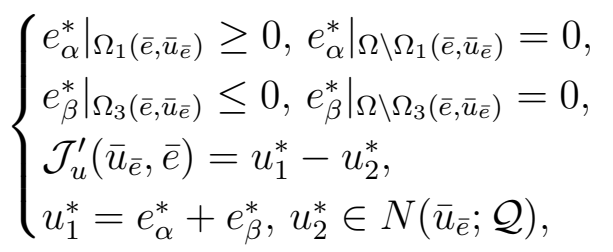

or, equivalently, as follows

$$
\left\{\begin{array}{l}
\left.e_{\alpha}^{*}\right|_{\Omega_{1}\left(\bar{e}, \bar{u}_{\bar{e}}\right)} \geq 0,\left.e_{\alpha}^{*}\right|_{\Omega \backslash \Omega_{1}\left(\bar{e}, \bar{u}_{\bar{e}}\right)}=0 \\
\left.e_{\beta}^{*}\right|_{\Omega_{3}\left(\bar{e}, \bar{u}_{\bar{e}}\right)} \leq 0,\left.e_{\beta}^{*}\right|_{\Omega \backslash \Omega_{3}\left(\bar{e}, \bar{u}_{\bar{e}}\right)}=0 \\
\varphi_{\bar{u}_{\bar{e}}, \bar{e}}+\zeta \bar{u}_{\bar{e}}=e_{\alpha}^{*}+e_{\beta}^{*}-u_{2}^{*}, \\
u_{2}^{*} \in N\left(\bar{u}_{\bar{e}} ; \mathcal{Q}\right)
\end{array}\right.
$$

Combining (3.13) with (3.14) we get

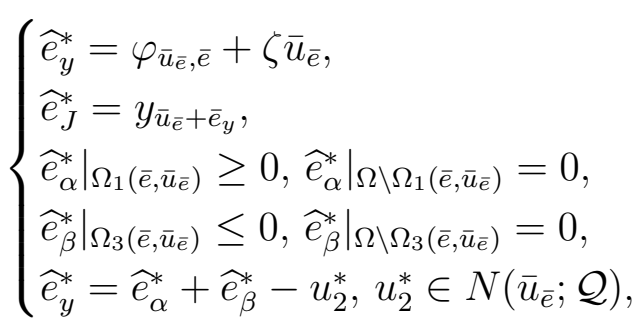

which yields (3.9).

If, in addition, $S: \operatorname{dom} \mathcal{G} \rightrightarrows L^{2}(\Omega)$ admits a local upper Lipschitzian selection at $\left(\bar{e}, \bar{u}_{\bar{e}}\right)$, then in the arguments above we use equality (3.3) instead of estimate (3.2) to deduce that condition (3.9) is necessary and sufficient for the inclusion $\widehat{e}^{*} \in \widehat{\partial} \mu(\bar{e})$.

\subsection{Limiting subgradients of marginal functions}

The next proposition provides us with an explicit formula for computing the Mordukhovich coderivative of the multifunction $\mathcal{G}(\cdot)$ that will be used to establish upper estimates for the Mordukhovich and the singular subdifferentials of the marginal function $\mu(\cdot)$.

Proposition 3.5. Assume that the assumptions (A1)-(A3) hold and let $\bar{u}_{\bar{e}} \in S(\bar{e})$. Then, for every $u^{*} \in L^{2}(\Omega)$, we have

$$
\begin{aligned}
D^{*} \mathcal{G}\left(\bar{e}, \bar{u}_{\bar{e}}\right)\left(u^{*}\right)= & \widehat{D}^{*} \mathcal{G}\left(\bar{e}, \bar{u}_{\bar{e}}\right)\left(u^{*}\right) \\
= & \left\{e^{*} \in E^{*} \mid e^{*}=\left(0,0, e_{\alpha}^{*}, e_{\beta}^{*}\right), u^{*}=u_{1}^{*}-u_{2}^{*},\right. \\
& u_{1}^{*}=e_{\alpha}^{*}+e_{\beta}^{*}, u_{2}^{*} \in N\left(\bar{u}_{\bar{e}} ; \mathcal{Q}\right) \\
& \left.e_{\alpha}^{*}\right|_{\Omega_{1}\left(\bar{e}, \bar{u}_{\bar{e}}\right)} \geq 0,\left.e_{\alpha}^{*}\right|_{\Omega \backslash \Omega_{1}\left(\bar{e}, \bar{u}_{\bar{e}}\right)}=0 \\
& \left.\left.e_{\beta}^{*}\right|_{\Omega_{3}\left(\bar{e}, \bar{u}_{\bar{e}}\right)} \leq 0,\left.e_{\beta}^{*}\right|_{\Omega \backslash \Omega_{3}\left(\bar{e}, \bar{u}_{\bar{e}}\right)}=0\right\}
\end{aligned}
$$

Proof. We observe that gph $\mathcal{G}$ is closed in the product space $E \times L^{2}(\Omega)$. By the definitions of coderivatives, we have $\widehat{D}^{*} \mathcal{G}\left(\bar{e}, \bar{u}_{\bar{e}}\right)\left(u^{*}\right) \subset D^{*} \mathcal{G}\left(\bar{e}, \bar{u}_{\bar{e}}\right)\left(u^{*}\right)$. Let us verify the opposite inclusion. 
Fix any $e^{*}=\left(e_{y}^{*}, e_{J}^{*}, e_{\alpha}^{*}, e_{\beta}^{*}\right) \in D^{*} \mathcal{G}\left(\bar{e}, \bar{u}_{\bar{e}}\right)\left(u^{*}\right)$. Then, by (2.16), (2.17), and (2.15), there exist sequences $\left(e_{n}, u_{n}\right) \in \operatorname{gph} \mathcal{G}$ and $\left(e_{n}^{*}, u_{n}^{*}\right) \in E^{*} \times L^{2}(\Omega)$ satisfying

$$
\left(e_{n}, u_{n}\right) \rightarrow\left(\bar{e}, \bar{u}_{\bar{e}}\right),\left(e_{n}^{*}, u_{n}^{*}\right) \stackrel{w^{*}}{\longrightarrow}\left(e^{*}, u^{*}\right), e_{n}^{*} \in \widehat{D}^{*} \mathcal{G}\left(e_{n}, u_{n}\right)\left(u_{n}^{*}\right), \forall n \in \mathbb{N}
$$

and

$$
\left(e_{n}, u_{n}\right) \rightarrow\left(\bar{e}, \bar{u}_{\bar{e}}\right) \text { pointwise a.e. on } \Omega \text {. }
$$

For every $n \in \mathbb{N}$, since $e_{n}^{*} \in \widehat{D}^{*} \mathcal{G}\left(e_{n}, u_{n}\right)\left(u_{n}^{*}\right)$, by (3.8) we infer that $e_{n}^{*}=\left(0,0,\left(e_{n}^{*}\right)_{\alpha},\left(e_{n}^{*}\right)_{\beta}\right)$ satisfies the following conditions

$$
\left\{\begin{array}{l}
u_{n}^{*}=\left(u_{n}^{*}\right)_{1}-\left(u_{n}^{*}\right)_{2}, \\
\left(u_{n}^{*}\right)_{1}=\left(e_{n}^{*}\right)_{\alpha}+\left(e_{n}^{*}\right)_{\beta},\left(u_{n}^{*}\right)_{2} \in N\left(u_{n} ; \mathcal{Q}\right), \\
\left.\left(e_{n}^{*}\right)_{\alpha}\right|_{\Omega_{1}\left(e_{n}, u_{n}\right)} \geq 0,\left.\left(e_{n}^{*}\right)_{\alpha}\right|_{\Omega \backslash \Omega_{1}\left(e_{n}, u_{n}\right)}=0, \\
\left.\left(e_{n}^{*}\right)_{\beta}\right|_{\Omega_{3}\left(e_{n}, u_{n}\right)} \leq 0,\left.\left(e_{n}^{*}\right)_{\beta}\right|_{\Omega \backslash \Omega_{3}\left(e_{n}, u_{n}\right)}=0 .
\end{array}\right.
$$

By $e_{n}^{*}=\left(0,0,\left(e_{n}^{*}\right)_{\alpha},\left(e_{n}^{*}\right)_{\beta}\right) \stackrel{w^{*}}{\longrightarrow} e^{*}$, we deduce that $e^{*}=\left(0,0, e_{\alpha}^{*}, e_{\beta}^{*}\right)$ with

$$
\left(e_{n}^{*}\right)_{\alpha} \stackrel{w^{*}}{\longrightarrow} e_{\alpha}^{*} \text { and }\left(e_{n}^{*}\right)_{\beta} \stackrel{w^{*}}{\longrightarrow} e_{\beta}^{*} \text {. }
$$

From this and (3.16) it follows that $e_{\alpha}^{*} \geq 0$ and $e_{\beta}^{*} \leq 0$ on $\Omega$. We show that $\left.e_{\alpha}^{*}\right|_{\Omega \backslash \Omega_{1}\left(\bar{e}, \bar{u}_{\bar{e}}\right)}=0$ and $\left.e_{\beta}^{*}\right|_{\Omega \backslash \Omega_{3}\left(\bar{e}, \bar{u}_{\bar{e}}\right)}=0$. Let $\varepsilon>0$ be given. Let $B \subset A_{\varepsilon}:=\left\{x \in \Omega \mid \bar{u} \geq \alpha+\bar{e}_{\alpha}+\varepsilon\right\} \subset$ $\Omega \backslash \Omega_{1}\left(\bar{e}, \bar{u}_{\bar{e}}\right)$ be a bounded set of positive measure. Since $\left(e_{n}^{*}\right)_{\alpha}=0$ on $\Omega \backslash \Omega_{1}\left(\bar{e}_{\bar{u}} \bar{u}_{\bar{e}}\right)$, we get

$$
\begin{aligned}
\left\langle e_{\alpha}^{*}, \chi_{B}\right\rangle & =\lim _{n \rightarrow \infty}\left\langle\left(e_{n}^{*}\right)_{\alpha}, \chi_{B}\right\rangle \\
& =\lim _{n \rightarrow \infty}\left\langle\left(e_{n}^{*}\right)_{\alpha},\left.\chi_{B}\right|_{\Omega \backslash \Omega_{1}\left(e_{n}, u_{n}\right)}+\left.\chi_{B}\right|_{\Omega_{1}\left(e_{n}, u_{n}\right)}\right\rangle \\
& =\lim _{n \rightarrow \infty}\left\langle\left(e_{n}^{*}\right)_{\alpha},\left.\chi_{B}\right|_{\Omega_{1}\left(e_{n}, u_{n}\right)}\right\rangle .
\end{aligned}
$$

Due to pointwise convergence, we have $\chi_{B} \chi_{\Omega_{1}\left(e_{n}, u_{n}\right)} \rightarrow 0$ pointwise almost everywhere. By dominated convergence theorem, $\chi_{B} \chi_{\Omega_{1}\left(e_{n}, u_{n}\right)} \rightarrow 0$ in $L^{2}(\Omega)$. Hence, $\lim _{n \rightarrow \infty}\left\langle\left(e_{n}^{*}\right)_{\alpha}, \chi_{B}\right\rangle=0$. It follows that $e_{\alpha}^{*}=0$ on $A_{\varepsilon}$ for all $\varepsilon>0$, which in turn implies $e_{\alpha}^{*}=0$ on $\Omega \backslash \Omega_{1}\left(\bar{e}, \bar{u}_{\bar{e}}\right)$. Similarly, we can prove $\left.e_{\beta}^{*}\right|_{\Omega \backslash \Omega_{3}\left(\bar{e}, \bar{u}_{\bar{e}}\right)}=0$. We have shown that

$$
\left\{\begin{array}{l}
\left.e_{\alpha}^{*}\right|_{\Omega_{1}\left(\bar{e}, \bar{u}_{\bar{e}}\right)} \geq 0,\left.e_{\alpha}^{*}\right|_{\Omega \backslash \Omega_{1}\left(\bar{e}, \bar{u}_{\bar{e}}\right)}=0 \\
\left.e_{\beta}^{*}\right|_{\Omega_{3}\left(\bar{e}, \bar{u}_{\bar{e}}\right)} \leq 0,\left.e_{\beta}^{*}\right|_{\Omega \backslash \Omega_{3}\left(\bar{e}, \bar{u}_{\bar{e}}\right)}=0 .
\end{array}\right.
$$

Since $u_{n} \rightarrow \bar{u}_{\bar{e}}$ with $u_{n} \in \mathcal{Q}$, we have $e_{\alpha}^{*}+e_{\beta}^{*}-u^{*} \in N\left(\bar{u}_{\bar{e}} ; \mathcal{Q}\right)$. Indeed, for all $v \in \mathcal{Q}$, due to

$$
\left(e_{n}^{*}\right)_{\alpha}+\left(e_{n}^{*}\right)_{\beta}-u_{n}^{*}=\left(u_{n}^{*}\right)_{2} \in N\left(u_{n} ; \mathcal{Q}\right)
$$

we obtain $\left\langle\left(e_{n}^{*}\right)_{\alpha}+\left(e_{n}^{*}\right)_{\beta}-u_{n}^{*}, v-u_{n}\right\rangle \leq 0$ for every $n \in \mathbb{N}$. In addition, since

$$
\left(e_{n}^{*}\right)_{\alpha}+\left(e_{n}^{*}\right)_{\beta}-u_{n}^{*} \stackrel{w^{*}}{\rightarrow} e_{\alpha}^{*}+e_{\beta}^{*}-u^{*} \quad \text { and } \quad v-u_{n} \rightarrow v-\bar{u}_{\bar{e}}
$$

we have

$$
\left\langle\left(e_{n}^{*}\right)_{\alpha}+\left(e_{n}^{*}\right)_{\beta}-u_{n}^{*}, v-u_{n}\right\rangle \rightarrow\left\langle e_{\alpha}^{*}+e_{\beta}^{*}-u^{*}, v-\bar{u}_{\bar{e}}\right\rangle .
$$

This implies that $\left\langle e_{\alpha}^{*}+e_{\beta}^{*}-u^{*}, v-\bar{u}_{\bar{e}}\right\rangle \leq 0$, which yields $e_{\alpha}^{*}+e_{\beta}^{*}-u^{*} \in N\left(\bar{u}_{\bar{e}} ; \mathcal{Q}\right)$. We now put

$$
u_{1}^{*}=e_{\alpha}^{*}+e_{\beta}^{*} \quad \text { and } \quad u_{2}^{*}=u_{1}^{*}-u^{*} \in N\left(\bar{u}_{\bar{e}} ; \mathcal{Q}\right) .
$$

From this and (3.17) we obtain $e^{*} \in \widehat{D}^{*} \mathcal{G}\left(\bar{e}, \bar{u}_{\bar{e}}\right)\left(u^{*}\right)$. Thus, $D^{*} \mathcal{G}\left(\bar{e}, \bar{u}_{\bar{e}}\right)\left(u^{*}\right) \subset \widehat{D}^{*} \mathcal{G}\left(\bar{e}, \bar{u}_{\bar{e}}\right)\left(u^{*}\right)$. We have shown that $D^{*} \mathcal{G}\left(\bar{e}, \bar{u}_{\bar{e}}\right)\left(u^{*}\right)=\widehat{D}^{*} \mathcal{G}\left(\bar{e}, \bar{u}_{\bar{e}}\right)\left(u^{*}\right)$ and (3.15) follows. 
The next two theorems establish respectively upper estimates for the Mordukhovich and the singular subdifferentials of the marginal function $\mu(\cdot)$.

Theorem 3.6. Assume that the assumptions (A1)-(A3) hold and let $\bar{u}_{\bar{e}} \in S(\bar{e})$. Then it is necessary for an element $e^{*}=\left(e_{y}^{*}, e_{J}^{*}, e_{\alpha}^{*}, e_{\beta}^{*}\right)$ from $E^{*}$ belonging to $\partial \mu(\bar{e})$ that

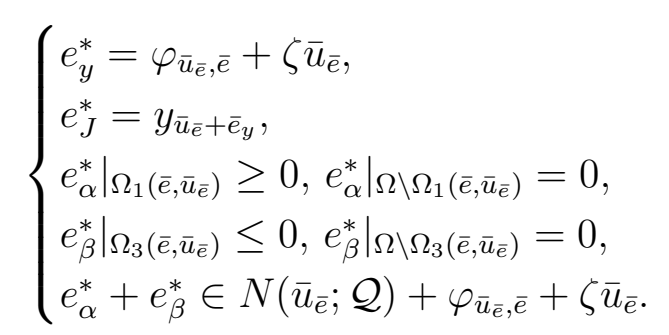

If, in addition, $S: \operatorname{dom} \mathcal{G} \rightrightarrows L^{2}(\Omega)$ admits a local upper Lipschitzian selection at $\left(\bar{e}, \bar{u}_{\bar{e}}\right)$, then the marginal function $\mu(\cdot)$ is lower regular at $\bar{e}$ and (3.18) is also sufficient for the inclusion $e^{*} \in \partial \mu(\bar{e})$.

Proof. By our assumptions, $\mathcal{J}(u, e)$ is continuously differentiable at $\left(\bar{u}_{\bar{e}}, \bar{e}\right)$, thus $\mathcal{J}(u, e)$ is strictly differentiable at $\left(\bar{u}_{\bar{e}}, \bar{e}\right)$ and Lipschitz continuous around $\left(\bar{u}_{\bar{e}}, \bar{e}\right)$. This implies that $\partial \mathcal{J}\left(\bar{u}_{\bar{e}}, \bar{e}\right)=\left\{\left(\mathcal{J}_{u}^{\prime}\left(\bar{u}_{\bar{e}}, \bar{e}\right), \mathcal{J}_{e}^{\prime}\left(\bar{u}_{\bar{e}}, \bar{e}\right)\right)\right\}$. Applying [21, Theorem 7(i)], we obtain

$$
\partial \mu(\bar{e}) \subset \mathcal{J}_{e}^{\prime}\left(\bar{u}_{\bar{e}}, \bar{e}\right)+D^{*} \mathcal{G}\left(\bar{e}, \bar{u}_{\bar{e}}\right)\left(\mathcal{J}_{u}^{\prime}\left(\bar{u}_{\bar{e}}, \bar{e}\right)\right) .
$$

By Proposition 3.5, we infer that $D^{*} \mathcal{G}\left(\bar{e}, \bar{u}_{\bar{e}}\right)\left(\mathcal{J}_{u}^{\prime}\left(\bar{u}_{\bar{e}}, \bar{e}\right)\right)=\widehat{D}^{*} \mathcal{G}\left(\bar{e}, \bar{u}_{\bar{e}}\right)\left(\mathcal{J}_{u}^{\prime}\left(\bar{u}_{\bar{e}}, \bar{e}\right)\right)$. From this and (3.19) we get

$$
\partial \mu(\bar{e}) \subset \mathcal{J}_{e}^{\prime}\left(\bar{u}_{\bar{e}}, \bar{e}\right)+\widehat{D}^{*} \mathcal{G}\left(\bar{e}, \bar{u}_{\bar{e}}\right)\left(\mathcal{J}_{u}^{\prime}\left(\bar{u}_{\bar{e}}, \bar{e}\right)\right)
$$

By (3.20) and by Theorem 3.4, we deduce that (3.18) is necessary for $e^{*} \in \partial \mu(\bar{e})$.

If, in addition, $S: \operatorname{dom} \mathcal{G} \rightrightarrows L^{2}(\Omega)$ admits a local upper Lipschitzian selection at $\left(\bar{e}, \bar{u}_{\bar{e}}\right)$, then by [21, Theorem 7(iii)] the marginal function $\mu(\cdot)$ is lower regular at $\bar{e}$ and (3.19) holds as equality

$$
\begin{aligned}
\partial \mu(\bar{e}) & =\mathcal{J}_{e}^{\prime}\left(\bar{u}_{\bar{e}}, \bar{e}\right)+D^{*} \mathcal{G}\left(\bar{e}, \bar{u}_{\bar{e}}\right)\left(\mathcal{J}_{u}^{\prime}\left(\bar{u}_{\bar{e}}, \bar{e}\right)\right) \\
& =\mathcal{J}_{e}^{\prime}\left(\bar{u}_{\bar{e}}, \bar{e}\right)+\widehat{D}^{*} \mathcal{G}\left(\bar{e}, \bar{u}_{\bar{e}}\right)\left(\mathcal{J}_{u}^{\prime}\left(\bar{u}_{\bar{e}}, \bar{e}\right)\right) .
\end{aligned}
$$

By (3.21) and by Theorem 3.4, condition (3.18) is also sufficient for $e^{*} \in \partial \mu(\bar{e})$.

Remark 3.7. From Theorems 3.4, 3.6 we see that the necessary conditions (3.9) and (3.18) coincide because $\mathcal{G}(\cdot)$ is normally regular at $\left(\bar{e}, \bar{u}_{\bar{e}}\right)$ by (3.15). These necessary conditions are also the same sufficient conditions provided that $S: \operatorname{dom} \mathcal{G} \rightrightarrows L^{2}(\Omega)$ admits a local upper Lipschitzian selection at $\left(\bar{e}, \bar{u}_{\bar{e}}\right)$, which yields $\widehat{\partial} \mu(\bar{e})=\partial \mu(\bar{e})$, i.e., the marginal function $\mu(\cdot)$ is lower regular at $\bar{e}$.

Theorem 3.8. Assume that the assumptions (A1)-(A3) hold and let $\bar{u}_{\bar{e}} \in S(\bar{e})$. Then, we have the following estimate

$$
\begin{gathered}
\partial^{\infty} \mu(\bar{e}) \subset\left\{\left(0,0, e_{\alpha}^{*}, e_{\beta}^{*}\right) \in E^{*} \mid e_{\alpha}^{*}+e_{\beta}^{*} \in N\left(\bar{u}_{\bar{e}} ; \mathcal{Q}\right),\right. \\
\left.e_{\alpha}^{*}\right|_{\Omega_{1}\left(\bar{e}, \bar{u}_{\bar{e}}\right)} \geq 0,\left.e_{\alpha}^{*}\right|_{\Omega \backslash \Omega_{1}\left(\bar{e}, \bar{u}_{\bar{e}}\right)}=0, \\
\left.\left.e_{\beta}^{*}\right|_{\Omega_{3}\left(\bar{e}, \bar{u}_{\bar{e}}\right)} \leq 0,\left.e_{\beta}^{*}\right|_{\Omega \backslash \Omega_{3}\left(\bar{e}, \bar{u}_{\bar{e}}\right)}=0\right\} .
\end{gathered}
$$

Proof. Our assumptions ensure that $\mathcal{J}(u, e)$ is Lipschitz continuous around $\left(\bar{u}_{\bar{e}}, \bar{e}\right)$. Hence, we have $\partial^{\infty} \mathcal{J}\left(\bar{u}_{\bar{e}}, \bar{e}\right)=\{(0,0)\}$. By [21, Theorem $\left.7(\mathrm{i})\right]$, we deduce that

$$
\partial^{\infty} \mu(\bar{e}) \subset D^{*} \mathcal{G}\left(\bar{e}, \bar{u}_{\bar{e}}\right)(0) .
$$

By (3.23), formula (3.22) follows directly from formula (3.15). 
Corollary 3.9. Assume that the assumptions (A1)-(A3) hold and let $\bar{u}_{\bar{e}} \in S(\bar{e})$. Then, the following hold:

(i) If $\bar{u}_{\bar{e}} \in \operatorname{int} \mathcal{Q}$, then we have

$$
\partial^{\infty} \mu(\bar{e}) \subset\{0\}
$$

(ii) If $\bar{u}_{\bar{e}} \in \operatorname{int} \mathcal{Q}$, and there exists a sequence $e_{n} \rightarrow \bar{e}$ such that $\bar{u}_{e_{n}} \rightarrow \bar{u}_{\bar{e}}$ in $L^{p_{0}}(\Omega)$ with $\bar{u}_{e_{n}} \in S\left(e_{n}\right)$ and $\widehat{\partial} \mu\left(e_{n}\right) \neq \emptyset$, then we have

$$
0 \in \partial^{\infty} \mu(\bar{e})
$$

Consequently, (3.24) holds as an equality.

Proof. (i) Take any $\left(0,0, e_{\alpha}^{*}, e_{\beta}^{*}\right) \in \partial^{\infty} \mu(\bar{e})$. Note that $N\left(\bar{u}_{\bar{e}} ; \mathcal{Q}\right)=\{0\}$ because $\bar{u}_{\bar{e}} \in \operatorname{int} \mathcal{Q}$. Hence, from (3.22) it follows that $e_{\alpha}^{*}=e_{\beta}^{*}=0$ a.e. on $\Omega$. This yields (3.24).

(ii) Choose $\lambda_{n}=\varepsilon_{n}=1 / n$ and take any $\widehat{e}_{n}^{*} \in \widehat{\partial} \mu\left(e_{n}\right) \subset \widehat{\partial}_{\varepsilon_{n}} \mu\left(e_{n}\right)$ for every $n \in \mathbb{N}$. Since $\widehat{e}_{n}^{*} \in \widehat{\partial} \mu\left(e_{n}\right), \widehat{e}_{n}^{*}$ holds (3.9) . Because $\bar{u}_{e_{n}} \rightarrow \bar{u}_{\bar{e}}$ and $\bar{u}_{\bar{e}} \in \operatorname{int} \mathcal{Q}$, we have $\bar{u}_{e_{n}} \in \operatorname{int} \mathcal{Q}$ for all $n$ large enough. Hence, $N\left(\bar{u}_{e_{n}} ; \mathcal{Q}\right)=\{0\}$ for all $n$ sufficiently large. Consequently, according to (3.9), $\widehat{e}_{n}^{*}$ must be bounded. Letting $n \rightarrow \infty$, we have

$$
e_{n} \rightarrow \bar{e}, \quad \mu\left(e_{n}\right) \rightarrow \mu(\bar{e}), \quad \varepsilon_{n} \downarrow 0, \quad \lambda_{n} \downarrow 0, \quad \lambda_{n} \widehat{e}_{n}^{*} \stackrel{w^{*}}{\longrightarrow} 0
$$

which yields $0 \in \partial^{\infty} \mu(\bar{e})$ by (2.13) . Combining this with (i) we obtain $\partial^{\infty} \mu(\bar{e})=\{0\}$.

Remark 3.10. If $\widehat{\partial} \mu(\bar{e}) \neq \emptyset$, then (3.25) holds without the assumption $\bar{u}_{\bar{e}} \in \operatorname{int} \mathcal{Q}$. Indeed, take any $\widehat{e}^{*} \in \widehat{\partial} \mu(\bar{e})$ and choose $e_{n}=\bar{e}, \lambda_{n}=\varepsilon_{n}=1 / n, \widehat{e}_{n}^{*}=\widehat{e}^{*}$ for every $n \in \mathbb{N}$. Letting $n \rightarrow \infty$, we obtain (3.26), which implies $0 \in \partial^{\infty} \mu(\bar{e})$.

\section{Parametric bang-bang control problems}

In this section, we consider the parametric control problem (2.3), where the functional $J(\cdot)$ is given in (2.1) with $\zeta=0$ a.e. on $\Omega, \mathcal{Q}=L^{p_{0}}(\Omega)$ with $p_{0}>N / 2$ while $q_{0}=s_{0}=1$, and $p_{1}=p_{2}=2, p_{3}=p_{4}=\infty$ in (2.6). In addition, the solution map $S: E \rightrightarrows L^{1}(\Omega)$ is defined by (2.8) with respect to $\mathcal{J}(u, e): L^{1}(\Omega) \times E \rightarrow \mathbb{R}$. For this setting, we rewrite problem (2.3) as follows

$$
\begin{cases}\text { Minimize } & \mathcal{J}(u, e)=J\left(u+e_{y}\right)+\left(e_{J}, y_{u+e_{y}}\right)_{L^{2}(\Omega)} \\ \text { subject to } & u \in \mathcal{U}_{a d}(e)\end{cases}
$$

where $y_{u+e_{y}}$ is the weak solution of (2.4), and the functional $J(\cdot)$ is defined by

$$
J(u)=\int_{\Omega} L\left(x, y_{u}(x)\right) d x .
$$

Note that we have $\mathcal{U}_{\text {ad }}(e) \subset L^{\infty}(\Omega)$ for every $e \in E=L^{2}(\Omega) \times L^{2}(\Omega) \times L^{\infty}(\Omega) \times L^{\infty}(\Omega)$.

In contrast to the previous section, the cost functional $\mathcal{J}: L^{1}(\Omega) \times E \rightarrow \mathbb{R}$ of problem (4.1) is not Fréchet differentiable. In addition, the problem of computing $\widehat{\partial}^{+} \mathcal{J}\left(\bar{u}_{\bar{e}}, \bar{e}\right)$ or checking $\widehat{\partial}^{+} \mathcal{J}\left(\bar{u}_{\bar{e}}, \bar{e}\right) \neq \emptyset$ at a given point $\left(\bar{u}_{\bar{e}}, \bar{e}\right) \in \operatorname{gph} S$ remains open. Therefore, we can not apply [21, Theorems 1 and 2] to compute/estimate subdifferentials of the marginal function $\mu(\cdot)$ of problem (4.1). For this reason, by the definition of regular subgradients we will establish directly a characterization of regular subgradients of the marginal function $\mu(\cdot)$ at a given point $\left(\bar{e}, \bar{u}_{\bar{e}}\right) \in \operatorname{gph} S$ in a subspace $E_{1}^{*}$ (see the definition of $E_{1}^{*}$ below) of $E^{*}$ via local upper 
Hölderian selections of the solution map $S: \operatorname{dom} \mathcal{G} \rightrightarrows L^{1}(\Omega)$ at the point $\left(\bar{e}, \bar{u}_{\bar{e}}\right)$. This leads to some lower estimates for the regular subdifferential of $\mu(\cdot)$ at $\left(\bar{e}, \bar{u}_{\bar{e}}\right)$.

Consider problem (2.1) with $\mathcal{U}_{a d}$ being replaced by $\mathcal{U}_{a d}(\bar{e})$ and let $\bar{u}_{\bar{e}} \in \mathcal{U}_{a d}(\bar{e})$ be a solution of problem (2.1) in the sense of $L^{p_{0}}(\Omega)$. From (2.28), we deduce that

$$
\bar{u}_{\bar{e}}(x)= \begin{cases}\left(\alpha+\bar{e}_{\alpha}\right)(x), & \text { if } \varphi_{\bar{u}_{\bar{e}}}(x)>0 \\ \left(\beta+\bar{e}_{\beta}\right)(x), & \text { if } \varphi_{\bar{u}_{\bar{e}}}(x)<0,\end{cases}
$$

and

$$
\varphi_{\bar{u}_{\bar{e}}}(x)\left\{\begin{array}{l}
\geq 0, \quad \text { if } \bar{u}_{\bar{e}}(x)=\left(\alpha+\bar{e}_{\alpha}\right)(x) \\
\leq 0, \quad \text { if } \bar{u}_{\bar{e}}(x)=\left(\beta+\bar{e}_{\beta}\right)(x) \\
=0, \quad \text { if } \bar{u}_{\bar{e}}(x) \in\left(\left(\alpha+\bar{e}_{\alpha}\right)(x),\left(\beta+\bar{e}_{\beta}\right)(x)\right) .
\end{array}\right.
$$

In general, solutions $\bar{u}_{\bar{e}}$ have the so-called bang-bang property: for a.a. $x \in \Omega$, it holds that $\bar{u}_{\bar{e}}(x) \in\left\{\left(\alpha+\bar{e}_{\alpha}\right)(x),\left(\beta+\bar{e}_{\beta}\right)(x)\right\}$. Consider the case where $\left\{x \in \Omega \mid \varphi_{\bar{u}_{\bar{e}}}(x)=0\right\}$ has a zero Lebesgue measure. Then, it follows from (4.2) and (4.3) that $\left.\bar{u}_{\bar{e}}(x) \in\left\{\alpha+\bar{e}_{\alpha}\right)(x),\left(\beta+\bar{e}_{\beta}\right)(x)\right\}$ for a.a. $x \in \Omega$, i.e., $\bar{u}_{\bar{e}}$ is a bang-bang control. In this section, we are interested in the last property of the reference control $\bar{u}_{\bar{e}}$.

\subsection{Local upper Hölderian selections of solution map}

According to [10], sufficient second-order optimality conditions for bang-bang controls $\bar{u}_{\bar{e}}$ of problem (2.1) with respect to the admissible control set $\mathcal{U}_{a d}(\bar{e})$ established under the following assumption (A4) posed on the adjoint state $\varphi_{\bar{u}_{\bar{e}}}$ in the case where $\left\{x \in \Omega \mid \varphi_{\bar{u}_{\bar{e}}}(x)=0\right\}$ has a zero Lebesgue measure.

(A4) Assume that $\bar{u}_{\bar{e}} \in \mathcal{U}_{a d}(\bar{e})$, and it satisfies the system (2.26)-(2.28) and the following condition

$$
\exists K>0, \exists æ>0 \text { such that }\left|\left\{x \in \Omega:\left|\varphi_{\bar{u}_{\bar{e}}}(x)\right| \leq \varepsilon\right\}\right| \leq K \varepsilon^{\infty}, \forall \varepsilon>0 .
$$

In (4.4), the notation $|\cdot|$ stands for the Lebesgue measure.

Proposition 4.1. (See [23, Proposition 3.2]) Assume that (A1)-(A4) hold at $\bar{u}_{\bar{e}} \in \mathcal{U}_{a d}(\bar{e})$. Then, there exists $\kappa>0$ such that

$$
J^{\prime}\left(\bar{u}_{\bar{e}}\right)\left(u-\bar{u}_{\bar{e}}\right) \geq \kappa\left\|u-\bar{u}_{\bar{e}}\right\|_{L^{1}(\Omega)}^{1+\frac{1}{x}}, \forall u \in \mathcal{U}_{a d}(\bar{e}) .
$$

For each $\bar{u}_{\bar{e}} \in \mathcal{U}_{a d}(\bar{e})$ and $\tau \geq 0,1 \leq p \leq \infty$, we define the cone

$$
C_{\bar{u}_{\bar{e}, p}}^{\tau}=\left\{v \in L^{p}(\Omega) \mid v(x)\left\{\begin{array}{ll}
\geq 0 & \text { if } \bar{u}_{\bar{e}}(x)=\left(\alpha+\bar{e}_{\alpha}\right)(x) \\
\leq 0 & \text { if } \bar{u}_{\bar{e}}(x)=\left(\beta+\bar{e}_{\beta}\right)(x) \\
=0 & \text { if }\left|\varphi_{\bar{u}_{\bar{e}}}(x)\right|>\tau
\end{array}\right\} .\right.
$$

The forthcoming theorem provides us with a second-order sufficient condition for bang-bang controls $\bar{u}_{\bar{e}} \in \mathcal{U}_{a d}(\bar{e})$ to be optimal for problem (4.1) with respect to $\bar{e} \in E$.

Theorem 4.2. (See [23, Theorem 3.1]) Let $\bar{u}_{\bar{e}} \in \mathcal{U}_{a d}(\bar{e})$ be a feasible bang-bang control for problem (4.1) satisfying (A1)-(A4). Assume that there exist $\delta>0$ and $\tau>0$ such that

$$
J^{\prime \prime}\left(\bar{u}_{\bar{e}}\right) v^{2} \geq \delta\left\|z_{v}\right\|_{L^{2}(\Omega)}^{2}, \forall v \in C_{\bar{u}_{\bar{e}}, 2}^{\tau},
$$

where $z_{v}=G^{\prime}\left(\bar{u}_{\bar{e}}\right) v$ is the solution of (2.21) for $y=y_{\bar{u}_{\bar{e}}}$. Then, there exists $\varepsilon>0$ such that

$$
J\left(\bar{u}_{\bar{e}}\right)+\frac{\kappa}{2}\left\|u-\bar{u}_{\bar{e}}\right\|_{L^{1}(\Omega)}^{1+\frac{1}{\infty}}+\frac{\delta}{8}\left\|z_{u-\bar{u}_{\bar{e}}}\right\|_{L^{2}(\Omega)}^{2} \leq J(u), \forall u \in \mathcal{U}_{a d}(\bar{e}) \cap \bar{B}_{\varepsilon}^{2}\left(\bar{u}_{\bar{e}}\right),
$$

with $z_{u-\bar{u}_{\bar{e}}}=G^{\prime}\left(\bar{u}_{\bar{e}}\right)\left(u-\bar{u}_{\bar{e}}\right)$ and $\kappa$ being given in Proposition 4.1 . 
In what follows, assumption (A4) and condition (4.7) will play a crucial role to prove the existence of local upper Hölderian selections of the solution map $S(\cdot)$.

Recall that a vector $v$ is an extremal point of a set $\Theta$ in a Banach space $X$ if and only if $v=\lambda v_{1}+(1-\lambda) v_{2}$ with $v_{1}, v_{2} \in \Theta$ and $0<\lambda<1$ entails $v_{1}=v_{2}=v$. We will denote the closed convex hull of $\Theta$ by $\overline{\operatorname{conv}} \Theta$.

Theorem 4.3. (See [32, Theorem 1]) Assume that $u_{n} \rightarrow u$ in $L^{1}(\Omega)$ and $u(x)$ is an extremal point of $\Theta(x):=\overline{\operatorname{conv}}\left(\left\{u_{n}(x)\right\}_{n \in \mathbb{N}} \cup\{u(x)\}\right)$ for a.a. $x \in \Omega$. Then, $u_{n} \rightarrow u$ in $L^{1}(\Omega)$.

We will use this theorem to lift weak convergence to strong convergence.

Lemma 4.4. Let $\bar{u}_{\bar{e}}$ be bang-bang, i.e., $\bar{u}_{\bar{e}}(x) \in\left\{\alpha(x)+\bar{e}_{\alpha}(x), \beta(x)+\bar{e}_{\beta}(x)\right\}$ for almost all $x \in \Omega$. Let $e_{n} \rightarrow \bar{e}$ in $E$ and choose $u_{n} \in \mathcal{U}_{a d}\left(e_{n}\right)$ such that $u_{n} \rightarrow \bar{u}_{\bar{e}}$ in $L^{1}(\Omega)$. Then $u_{n} \rightarrow \bar{u}_{\bar{e}}$ in $L^{1}(\Omega)$.

Proof. On the active set $\Omega_{1}\left(\bar{e}, \bar{u}_{\bar{e}}\right)$ it holds $\bar{u}_{\bar{e}}=\alpha+\bar{e}_{\alpha}$, cf., (3.4), which implies $u_{n}-\bar{u}_{\bar{e}}-$ $\left(\left(e_{\alpha}\right)_{n}-\bar{e}_{\alpha}\right) \geq 0$ on this subset. In addition, $u_{n}-\bar{u}_{\bar{e}}-\left(\left(e_{\alpha}\right)_{n}-\bar{e}_{\alpha}\right) \rightarrow 0$ in $L^{1}(\Omega)$. Then by Theorem 4.3 we conclude $u_{n}-\bar{u}_{\bar{e}}-\left(\left(e_{\alpha}\right)_{n}-\bar{e}_{\alpha}\right) \rightarrow 0$ in $L^{1}\left(\Omega_{1}\left(\bar{e}, \bar{u}_{\bar{e}}\right)\right)$, which implies $u_{n} \rightarrow \bar{u}_{\bar{e}}$ in $L^{1}\left(\Omega_{1}\left(\bar{e}, \bar{u}_{\bar{e}}\right)\right)$. Similarly, we find $u_{n} \rightarrow \bar{u}_{\bar{e}}$ in $L^{1}\left(\Omega_{3}\left(\bar{e}, \bar{u}_{\bar{e}}\right)\right)$. Since $\bar{u}_{\bar{e}}$ is bang-bang, it holds $\Omega=\Omega_{1}\left(\bar{e}, \bar{u}_{\bar{e}}\right) \cup \Omega_{3}\left(\bar{e}, \bar{u}_{\bar{e}}\right)$, which proves the claim.

A straightforward application of the above Theorem 4.3 would require to assume that $\bar{u}_{\bar{e}}(x)$ is an extremal point of the set $\overline{\operatorname{conv}}\left(\left\{u_{n}(x)\right\}_{n \in \mathbb{N}} \cup\left\{\bar{u}_{\bar{e}}(x)\right\}\right)$ for a.a. $x \in \Omega$. This cannot be guaranteed as the control bounds are perturbed, so $\bar{u}_{\bar{e}}(x)=\alpha(x)+\bar{e}_{\alpha}(x)$ does not imply $\bar{u}_{\bar{e}}(x) \leq u_{n}(x)$.

Note that similarly to Theorem 3.1, under the assumptions (A1)-(A3) we can show that for any $\bar{u}_{\bar{e}} \in S(\bar{e})$ and for every $e \in E$ near $\bar{e}$ enough the problem of minimizing the cost functional $\mathcal{J}(u, e)$ subject to $u \in \mathcal{U}_{a d}(e) \cap \bar{B}_{\varepsilon}^{p_{0}}\left(\bar{u}_{\bar{e}}\right)$ has at least one global solution $\bar{u}_{e}$, where $\bar{B}_{\varepsilon}^{p_{0}}\left(\bar{u}_{\bar{e}}\right)$ is the closed ball of center $\bar{u}_{\bar{e}}$ and radius $\varepsilon>0$ in $L^{p_{0}}(\Omega)$.

Theorem 4.5. Assume that (A1)-(A3) hold and let $\bar{u}_{\bar{e}} \in \mathcal{U}_{a d}(\bar{e})$ be a bang-bang solution of problem (4.1) with respect to $\bar{e} \in E$ such that $\bar{u}_{\bar{e}}$ is strict in some neighborhood $\bar{B}_{\varepsilon}^{p_{0}}\left(\bar{u}_{\bar{e}}\right)$ with $\varepsilon>0$. For every $e \in E$ near $\bar{e}$ enough, let $\bar{u}_{e}$ be a solution of the following control problem

$$
\text { Minimize } \mathcal{J}(u, e) \quad \text { subject to } \quad u \in \mathcal{U}_{a d}(e) \cap \bar{B}_{\varepsilon}^{p_{0}}\left(\bar{u}_{\bar{e}}\right),
$$

where $\mathcal{J}(\cdot, \cdot)$ is the cost functional of problem (4.1). Then, we have $\bar{u}_{e} \rightarrow \bar{u}_{\bar{e}}$ in $L^{p_{0}}(\Omega)$ when $e \rightarrow \bar{e}$ in $E$.

Proof. Let $\left\{e_{n}\right\}_{n \in \mathbb{N}}$ be such that $e_{n} \rightarrow \bar{e}$ in $E$ and let $\bar{u}_{e_{n}} \in \mathcal{U}_{a d}\left(e_{n}\right) \cap \bar{B}_{\varepsilon}^{p_{0}}\left(\bar{u}_{\bar{e}}\right)$ be a global solution of problem (4.9) with respect to $e_{n}$. Since the sequence $\left\{\bar{u}_{e_{n}}\right\}$ is bounded in $L^{p_{0}}(\Omega)$, it has a subsequence $\left\{\bar{u}_{e_{n_{k}}}\right\}$ with $\bar{u}_{e_{n_{k}}} \rightarrow \widehat{u}$ in $L^{p_{0}}(\Omega)$ for some $\widehat{u} \in \mathcal{U}_{a d}(\bar{e}) \cap \bar{B}_{\varepsilon}^{p_{0}}\left(\bar{u}_{\bar{e}}\right)$. Because $\bar{u}_{\bar{e}} \in \mathcal{U}_{a d}(\bar{e})$, we have

$$
\bar{u}_{\bar{e}}=\lambda\left(\alpha+\bar{e}_{\alpha}\right)+(1-\lambda)\left(\beta+\bar{e}_{\beta}\right)
$$

for some $\lambda(x) \in[0,1]$ for almost all $x \in \Omega$. Defining $u_{e_{n_{k}}} \in \mathcal{U}_{a d}\left(e_{n_{k}}\right) \cap \bar{B}_{\varepsilon}^{p_{0}}\left(\bar{u}_{\bar{e}}\right)$ by

$$
u_{e_{n_{k}}}:=\lambda\left(\alpha+\left(e_{n_{k}}\right)_{\alpha}\right)+(1-\lambda)\left(\beta+\left(e_{n_{k}}\right)_{\beta}\right)
$$

for almost all $x \in \Omega$, we have

$$
\left\|u_{e_{n_{k}}}-\bar{u}_{\bar{e}}\right\|_{L^{\infty}(\Omega)} \leq\left\|\left(e_{n_{k}}\right)_{\alpha}-\bar{e}_{\alpha}\right\|_{L^{\infty}(\Omega)}+\left\|\left(e_{n_{k}}\right)_{\beta}-\bar{e}_{\beta}\right\|_{L^{\infty}(\Omega)} \leq\left\|e_{n_{k}}-\bar{e}\right\|_{E} .
$$

It follows that when $k \rightarrow \infty$, we have

$$
\left\|u_{e_{n_{k}}}-\bar{u}_{\bar{e}}\right\|_{L^{p_{0}}(\Omega)} \leq|\Omega|^{1 / p_{0}}\left\|e_{n_{k}}-\bar{e}\right\|_{E} \rightarrow 0 .
$$


Letting $k \rightarrow \infty$, we get $\mathcal{J}\left(\bar{u}_{e_{n_{k}}}, e_{n_{k}}\right) \rightarrow \mathcal{J}(\widehat{u}, \bar{e})$ and $\mathcal{J}\left(u_{e_{n_{k}}}, e_{n_{k}}\right) \rightarrow \mathcal{J}\left(\bar{u}_{\bar{e}}, \bar{e}\right)$ with

$$
\mathcal{J}\left(\bar{u}_{e_{n_{k}}}, e_{n_{k}}\right) \leq \mathcal{J}\left(u_{e_{n_{k}}}, e_{n_{k}}\right), \forall k \in \mathbb{N} .
$$

This yields $\mathcal{J}(\widehat{u}, \bar{e}) \leq \mathcal{J}\left(\bar{u}_{\bar{e}}, \bar{e}\right)$. Therefore, we obtain $\widehat{u}=\bar{u}_{\bar{e}}$ since $\bar{u}_{\bar{e}}$ is a strict local solution of problem (4.1) with respect to $\bar{e}$. We have shown that $\bar{u}_{e_{n_{k}}} \rightarrow \bar{u}_{\bar{e}}$ in $L^{p_{0}}(\Omega)$. Consequently, $\bar{u}_{e_{n_{k}}} \rightarrow \bar{u}_{\bar{e}}$ in $L^{1}(\Omega)$. Since $\bar{u}_{\bar{e}}$ is bang-bang, we deduce $\bar{u}_{e_{n_{k}}} \rightarrow \bar{u}_{\bar{e}}$ in $L^{1}(\Omega)$ by Lemma 4.4. Note that $\bar{u}_{e_{n_{k}}} \in \mathcal{U}_{a d}\left(e_{n_{k}}\right)$ and the set $\bigcup_{k=1}^{\infty} \mathcal{U}_{a d}\left(e_{n_{k}}\right)$ is bounded in $L^{\infty}(\Omega)$. Hence, we can find a constant $M>0$ such that $\left\|\bar{u}_{e_{n_{k}}}-\bar{u}_{\bar{e}}\right\|_{L^{\infty}(\Omega)} \leq M$ for every $k \in \mathbb{N}$. Applying Hölder's inequality we get

$$
\left\|\bar{u}_{e_{n_{k}}}-\bar{u}_{\bar{e}}\right\|_{L^{p_{0}(\Omega)}} \leq\left\|\bar{u}_{e_{n_{k}}}-\bar{u}_{\bar{e}}\right\|_{L^{1}(\Omega)}^{1 / p_{0}}\left\|\bar{u}_{e_{n_{k}}}-\bar{u}_{\bar{e}}\right\|_{L^{\infty}(\Omega)}^{\left(p_{0}-1\right) / p_{0}} \leq M\left\|\bar{u}_{e_{n_{k}}}-\bar{u}_{\bar{e}}\right\|_{L^{1}(\Omega)}^{1 / p_{0}} \rightarrow 0,
$$

which verifies that $\bar{u}_{e} \rightarrow \bar{u}_{\bar{e}}$ in $L^{p_{0}}(\Omega)$ when $e \rightarrow \bar{e}$ in $E$.

Corollary 4.6. Assume that (A1)-(A3) hold and let $\bar{u}_{\bar{e}}$ be a unique bang-bang solution of problem (4.1) with respect to $\bar{e} \in E$. For every $e \in E$, let $\bar{u}_{e}$ be a solution of problem (4.1). Then, we have $\bar{u}_{e} \rightarrow \bar{u}_{\bar{e}}$ in $L^{p_{0}}(\Omega)$ when $e \rightarrow \bar{e}$ in $E$.

Proof. The proof is similar to the proof of Theorem 4.5, where the neighborhood $\bar{B}_{\varepsilon}^{p_{0}}\left(\bar{u}_{\bar{e}}\right)$ is replaced by $L^{p_{0}}(\Omega)$.

We need the following lemmas that will be used in the proofs of Hölderian stability for solutions to problem (4.1) in the parameter $e \in E$ as well as existence of Hölderian selections of the solution map $S(\cdot)$.

Lemma 4.7. Given $\widetilde{e} \in E$, let any $\widetilde{u} \in \mathcal{U}_{a d}(\widetilde{e})$ be given. Then, there exists $C_{1}=C_{1}(\widetilde{u}, \widetilde{e})>0$ such that

$$
\left\|y_{u}-y_{\widetilde{u}}\right\|_{Y}+\left\|\varphi_{u}-\varphi_{\widetilde{u}}\right\|_{Y} \leq C_{1}\|u-\widetilde{u}\|_{L^{p_{0}(\Omega)}}, \forall u \in L^{p_{0}}(\Omega),
$$

where $y_{u}$ and $\varphi_{u}$ are respectively the weak solutions of (2.2) and (2.25).

Proof. The proof is similar to the proof of [23, Lemma 4.1].

Lemma 4.8. Given $\widetilde{e} \in E$, let any $\widetilde{u} \in \mathcal{U}_{a d}(\widetilde{e})$ be given. Then, for every $\varepsilon>0$, there exists $\rho>0$ such that for $u \in \mathcal{U}_{a d}(\widetilde{e})$ with $\|u-\widetilde{u}\|_{L^{p_{0}(\Omega)}} \leq \rho$ the following holds

$$
\left|\mathcal{J}_{u}^{\prime \prime}(u, \widetilde{e}) v^{2}-\mathcal{J}_{u}^{\prime \prime}(\widetilde{u}, \widetilde{e}) v^{2}\right| \leq \varepsilon\left\|z_{v}\right\|_{L^{2}(\Omega)}^{2} \quad \forall v \in L^{p_{0}}(\Omega),
$$

where $z_{v}$ solves the linearized equation

$$
\left\{\begin{aligned}
A z_{u, v}+\frac{\partial f}{\partial y}\left(x, y_{\widetilde{u}+\widetilde{e}_{y}}\right) z_{u, v} & =v & & \text { in } \Omega \\
z_{u, v} & =0 & & \text { on } \Gamma .
\end{aligned}\right.
$$

Proof. The proof is similar to the proof of [9, Lemma 2.7].

Lemma 4.9. Let $\bar{e} \in E$ and $\eta>0$ be given. Then there is a constant $K_{M}>0$ such that

$$
\left|\mathcal{J}_{u}^{\prime \prime}(u, e)(v, w)\right| \leq K_{M}\left\|z_{u, v}^{e}\right\|_{L^{2}(\Omega)}\left\|z_{u, w}^{e}\right\|_{L^{2}(\Omega)}
$$

holds for all $e \in B_{\eta}(\bar{e}), u \in \mathcal{U}_{a d}(e), v, w \in L^{2}(\Omega)$, where $z_{u, v}^{e}=G^{\prime}\left(u+e_{y}\right) v, z_{u, w}^{e}=G^{\prime}\left(u+e_{y}\right) w$ solve the linearized equation

$$
\left\{\begin{aligned}
A z+\frac{\partial f}{\partial y}\left(x, y_{u+e_{y}}\right) z & =v & & \text { in } \Omega \\
z & =0 & & \text { on } \Gamma .
\end{aligned}\right.
$$


Proof. Let us put the set $\mathcal{U}=\bigcup_{e \in \bar{B}_{\eta}(\bar{e})} \mathcal{U}_{a d}(e)$. Let us define for $e \in B_{\eta}(\bar{e})$ and $u \in \mathcal{U}_{a d}(e)$ the function

$$
F(u, e)=\frac{\partial^{2} L}{\partial y^{2}}\left(x, y_{u+e_{y}}\right)-\varphi_{u, e} \frac{\partial^{2} f}{\partial y^{2}}\left(x, y_{u+e_{y}}\right) .
$$

Then $F$ is well-defined due to the assumptions posed on the functions $f$ and $L$. In addition, there is a constant $M>0$ such that

$$
\left\|y_{u+e_{y}}\right\|_{L^{\infty}(\Omega)}+\left\|\varphi_{u, e}\right\|_{L^{\infty}(\Omega)} \leq M
$$

holds for all $e \in B_{\eta}(\bar{e})$ and $u \in \mathcal{U}_{a d}(e)$. In addition, we can find a constant $\ell_{M}>0$ satisfying the condition

$$
\|F(u, e)\|_{L^{\infty}(\Omega)} \leq \ell_{M}
$$

for all $e \in B_{\eta}(\bar{e})$ and $u \in \mathcal{U}_{a d}(e)$. Consequently, for every $v, w \in L^{2}(\Omega)$ and $u \in \mathcal{U}$, it holds that

$$
\left|J^{\prime \prime}\left(u+e_{y}\right)(v, w)\right|=\left|\int_{\Omega} F(u, e) z_{u, v}^{e} z_{u, w}^{e} d x\right| \leq \ell_{M}\left\|z_{u, v}^{e}\right\|_{L^{2}(\Omega)}\left\|z_{u, w}^{e}\right\|_{L^{2}(\Omega)} .
$$

Note that $G^{\prime \prime}\left(u+e_{y}\right)(v, w)$ is the weak solution of (2.22) with respect to $y=G\left(u+e_{y}\right)$ and it satisfies the condition for some constant $C \geq 0$ as follows

$$
\begin{aligned}
\left\|G^{\prime \prime}\left(u+e_{y}\right)(v, w)\right\|_{L^{2}(\Omega)} & \leq C\left\|-\frac{\partial^{2} f}{\partial y^{2}}\left(x, y_{u+e_{y}}\right) z_{u, v}^{e} z_{u, w}^{e}\right\|_{L^{2}(\Omega)} \\
& \leq C\left\|\frac{\partial^{2} f}{\partial y^{2}}\left(x, y_{u+e_{y}}\right)\right\|_{L^{\infty}(\Omega)}\left\|z_{u, v}^{e}\right\|_{L^{2}(\Omega)}\left\|z_{u, w}^{e}\right\|_{L^{2}(\Omega)} \\
& \leq C C_{f, M}\left\|z_{u, v}^{e}\right\|_{L^{2}(\Omega)}\left\|z_{u, w}^{e}\right\|_{L^{2}(\Omega)},
\end{aligned}
$$

where $z_{u, v}^{e}=z_{u+e_{y}, v}=G^{\prime}\left(u+e_{y}\right) v$ and $z_{u, w}^{e}=z_{u+e_{y}, w}=G^{\prime}\left(u+e_{y}\right) w$. From (4.12), (4.13), and (4.1) it follows that

$$
\begin{aligned}
\left|\mathcal{J}_{u}^{\prime \prime}(u, e)(v, w)\right| & =\left|J^{\prime \prime}\left(u+e_{y}\right)(v, w)+\left(e_{J}, G^{\prime \prime}\left(u+e_{y}\right)(v, w)\right)_{L^{2}(\Omega)}\right| \\
& \leq\left|J^{\prime \prime}\left(u+e_{y}\right)(v, w)\right|+\left\|e_{J}\right\|_{L^{2}(\Omega)}\left\|G^{\prime \prime}\left(u+e_{y}\right)(v, w)\right\|_{L^{2}(\Omega)} \\
& \leq \ell_{M}\left\|z_{u, v}^{e}\right\|_{L^{2}(\Omega)}\left\|z_{u, w}^{e}\right\|_{L^{2}(\Omega)}+\eta C C_{f, M}\left\|z_{u, v}^{e}\right\|_{L^{2}(\Omega)}\left\|z_{u, w}^{e}\right\|_{L^{2}(\Omega)} \\
& =\left(\ell_{M}+\eta C C_{f, M}\right)\left\|z_{u, v}^{e}\right\|_{L^{2}(\Omega)}\left\|z_{u, w}^{e}\right\|_{L^{2}(\Omega)},
\end{aligned}
$$

which yields (4.11) with $K_{M}=\ell_{M}+\eta C C_{f, M}$.

Lemma 4.10. Let $\bar{e} \in E, \bar{u} \in \mathcal{U}_{a d}(\bar{e})$, and $\eta>0$ be given. Then there is a constant $K_{M}>0$ such that

$$
\left\|\varphi_{u, \bar{e}}-\varphi_{\bar{u}, \bar{e}}\right\|_{L^{\infty}(\Omega)} \leq K_{M}\|u-\bar{u}\|_{L^{1}(\Omega)}
$$

holds for all $e \in B_{\eta}(\bar{e}), u \in \mathcal{U}_{a d}(e)$.

Proof. The proof is similar to the proof of [10, Lemma 2.6].

Lemma 4.11. Let $\bar{e} \in E, \bar{u} \in \mathcal{U}_{a d}(\bar{e})$, and $\eta>0$ be given. Then there is a constant $K_{M}>0$ such that

$$
\left\|\varphi_{\bar{u}, e}-\varphi_{\bar{u}, \bar{e}}\right\|_{L^{\infty}(\Omega)} \leq K_{M}\|e-\bar{e}\|_{E}
$$

holds for all $e \in B_{\eta}(\bar{e})$. 
Proof. Since $\varphi_{\bar{u}, e}$ and $\varphi_{\bar{u}, \bar{e}}$ are the weak solutions of (2.30) with respect to $e$ and $\bar{e}$. Thus, we have

$$
\left\{\begin{array}{crl}
A^{*}\left(\varphi_{\bar{u}, e}-\varphi_{\bar{u}, \bar{e}}\right)+\frac{\partial f}{\partial y}\left(x, y_{\bar{u}+e_{y}}\right)\left(\varphi_{\bar{u}, e}-\varphi_{\bar{u}, \bar{e}}\right)=\frac{\partial L}{\partial y}\left(x, y_{\bar{u}+e_{y}}\right)-\frac{\partial L}{\partial y}\left(x, y_{\bar{u}+\bar{e}_{y}}\right) & \\
-\left(\frac{\partial f}{\partial y}\left(x, y_{\bar{u}+e_{y}}\right)-\frac{\partial f}{\partial y}\left(x, y_{\bar{u}+\bar{e}_{y}}\right)\right) \varphi_{\bar{u}, \bar{e}}+e_{J}-\bar{e}_{J} & \text { in } \Omega \\
\varphi_{\bar{u}, e}-\varphi_{\bar{u}, \bar{e}}=0 & \text { on } \Gamma,
\end{array}\right.
$$

By our assumptions, there exist $C>0, \ell_{1}>0, \ell_{2}>0$ such that

$$
\begin{aligned}
\left\|\varphi_{\bar{u}, e}-\varphi_{\bar{u}, \bar{e}}\right\|_{L^{\infty}(\Omega)} \leq & C\left(\left\|\frac{\partial L}{\partial y}\left(\cdot, y_{\bar{u}+e_{y}}\right)-\frac{\partial L}{\partial y}\left(\cdot, y_{\bar{u}+\bar{e}_{y}}\right)\right\|_{L^{2}(\Omega)}\right. \\
& \left.+\left\|\frac{\partial f}{\partial y}\left(\cdot, y_{\bar{u}+e_{y}}\right)-\frac{\partial f}{\partial y}\left(\cdot, y_{\bar{u}+\bar{e}_{y}}\right)\right\|_{L^{2}(\Omega)}\left\|\varphi_{\bar{u}, \bar{e}}\right\|_{L^{2}(\Omega)}+\left\|e_{J}-\bar{e}_{J}\right\|_{L^{2}(\Omega)}\right) \\
\leq & C\left(\ell_{1}\left\|y_{\bar{u}+e_{y}}-y_{\bar{u}+\bar{e}_{y}}\right\|_{L^{2}(\Omega)}+\left\|e_{J}-\bar{e}_{J}\right\|_{L^{2}(\Omega)}\right) \\
\leq & C\left(\ell_{1} \ell_{2}\left\|e_{y}-\bar{e}_{y}\right\|_{L^{2}(\Omega)}+\left\|e_{J}-\bar{e}_{J}\right\|_{L^{2}(\Omega)}\right) \\
\leq & K_{M}\|e-\bar{e}\|_{E},
\end{aligned}
$$

where $K_{M}:=C \max \left\{\ell_{1} \ell_{2}, 1\right\}$.

Lemma 4.12. Let $\bar{u} \in \mathcal{U}_{a d}(\bar{e})$ and $\rho>0$ be given. Then, there exists $c>0$ such that for all $\widehat{u} \in L^{\infty}(\Omega)$ with $\|\widehat{u}-\bar{u}\|_{L^{2}(\Omega)}<\rho$ it holds

$$
\left\|z_{\widehat{u}, w}^{\bar{e}}\right\|_{L^{2}(\Omega)} \leq c\|w\|_{L^{1}(\Omega)}, \forall w \in L^{2}(\Omega),
$$

and

$$
\left\|z_{\bar{u}, v}^{\bar{e}}-z_{\widehat{u}, v}^{\bar{e}}\right\|_{L^{2}(\Omega)} \leq c\left\|z_{\bar{u}, v}^{\bar{e}}\right\|_{L^{2}(\Omega)}\left\|\widehat{u}-\bar{u}_{\bar{e}}\right\|_{L^{2}(\Omega)},
$$

where we define $z_{\widehat{u}, w}^{\bar{e}}:=z_{\widehat{u}+\bar{e}_{y}, w}=G^{\prime}\left(\widehat{u}+\bar{e}_{y}\right) w$, and similarly for $z_{\bar{u}, v}^{\bar{e}}$ and $z_{\widehat{u}, v}^{\bar{e}}$.

Proof. It follows from [23, Lemma 4.2].

We also need the following extension of Proposition 4.1 to perturbed feasible sets.

Lemma 4.13. Assume that (A4) holds at $\bar{u}_{\bar{e}}$. Assume further that there is $\sigma>0$ such that

$$
\beta+\bar{e}_{\beta}-\left(\alpha+\bar{e}_{\alpha}\right) \geq \sigma \text { a.e. on } \Omega \text {. }
$$

Take $0<\eta<\sigma / 4$. Let $e \in B_{\eta}(\bar{e})$ and let $\bar{u}_{e} \in \mathcal{U}_{a d}(e)$ satisfy the first-order optimality system (2.29) -(2.31). Then there are $c>0$ and $\kappa^{\prime}>0$ independent of $e$ and $u_{e} \in \mathcal{U}_{a d}(\bar{e})$ such that

$$
\begin{aligned}
& \left(\mathcal{J}_{u}^{\prime}\left(\bar{u}_{e}, e\right)-\mathcal{J}_{u}^{\prime}\left(\bar{u}_{\bar{e}}, \bar{e}\right)\right)\left(\bar{u}_{\bar{e}}-\bar{u}_{e}\right) \\
& \quad \geq \kappa^{\prime}\left\|\bar{u}_{e}-\bar{u}_{\bar{e}}\right\|_{L^{1}(\Omega)}^{1+\frac{1}{\alpha}}+\frac{1}{2} \mathcal{J}_{u}^{\prime}\left(\bar{u}_{\bar{e}}, \bar{e}\right)\left(u_{e}-\bar{u}_{\bar{e}}\right) \\
& \quad-c\left(\|e-\bar{e}\|_{E}^{\frac{1}{x}}+\left\|\varphi_{\bar{u}_{e}, e}-\varphi_{\bar{u}_{\bar{e}}, \bar{e}}\right\|_{L^{\infty}(\Omega)}+\left\|\bar{u}_{e}-\bar{u}_{\bar{e}}\right\|_{L^{1}(\Omega)}\right)\|e-\bar{e}\|_{E}
\end{aligned}
$$

and $\left\|\bar{u}_{e}-u_{e}\right\|_{L^{\infty}(\Omega)} \leq\|e-\bar{e}\|_{E}$.

Proof. Due to the perturbation in the feasible set, we have $\bar{u}_{\bar{e}} \notin \mathcal{U}_{a d}(e)$ and $\bar{u}_{e} \notin \mathcal{U}_{a d}(\bar{e})$ in general. First, we construct controls $u_{e} \approx \bar{u}_{e}$ with $u_{e} \in \mathcal{U}_{a d}(\bar{e})$ and $u_{\bar{e}} \approx \bar{u}_{\bar{e}}$ with $u_{\bar{e}} \in \mathcal{U}_{a d}(e)$. Let us define

$$
\Omega_{\sigma}:=\left\{x \in \Omega|| \bar{u}_{e}-\bar{u}_{\bar{e}} \mid<\frac{\sigma}{2}\right\}
$$


Then on $\Omega_{\sigma}$ we have the implications

$$
\bar{u}_{\bar{e}}=\alpha+\bar{e}_{\alpha} \Longrightarrow u_{e} \leq \beta+e_{\beta}-\frac{\sigma}{4},
$$

and

$$
\bar{u}_{\bar{e}}=\beta+\bar{e}_{\beta} \Longrightarrow u_{e} \geq \alpha+e_{\alpha}+\frac{\sigma}{4}
$$

Let us define

$$
u_{e}:=\chi_{\Omega_{\sigma} \cap \Omega_{1}\left(\bar{u}_{\bar{e}}, \bar{e}\right)}\left(\bar{u}_{e}-\left(e_{\alpha}-\bar{e}_{\alpha}\right)\right)+\chi_{\Omega_{\sigma} \cap \Omega_{3}\left(\bar{u}_{\bar{e}}, \bar{e}\right)}\left(\bar{u}_{e}-\left(e_{\beta}-\bar{e}_{\beta}\right)\right)+\chi_{\Omega \backslash \Omega_{\sigma}} \operatorname{proj}_{\mathcal{U}_{a d}(\bar{e})}\left(\bar{u}_{e}\right),
$$

and

$$
u_{\bar{e}}:=\chi_{\Omega_{\sigma} \cap \Omega_{1}\left(\bar{u}_{\bar{e}}, \bar{e}\right)}\left(\bar{u}_{\bar{e}}+\left(e_{\alpha}-\bar{e}_{\alpha}\right)\right)+\chi_{\Omega_{\sigma} \cap \Omega_{3}\left(\bar{u}_{\bar{e}}, \bar{e}\right)}\left(\bar{u}_{\bar{e}}+\left(e_{\beta}-\bar{e}_{\beta}\right)\right)+\chi_{\Omega \backslash \Omega_{\sigma}} \operatorname{proj}_{\mathcal{U}_{a d}(e)}\left(\bar{u}_{\bar{e}}\right) .
$$

Due to the definition of $\Omega_{\sigma}$ it holds $u_{e} \in \mathcal{U}_{a d}(\bar{e})$ and $u_{\bar{e}} \in \mathcal{U}_{a d}(e)$. In addition, we have the important relation

$$
u_{e}-\bar{u}_{e}=-\left(u_{\bar{e}}-\bar{u}_{\bar{e}}\right) \text { on } \Omega_{\sigma} .
$$

Since the projection is Lipschitz continuous with respect to changes of upper and lower bounds, we have

$$
\left\|u_{e}-\bar{u}_{e}\right\|_{L^{\infty}(\Omega)},\left\|u_{\bar{e}}-\bar{u}_{\bar{e}}\right\|_{L^{\infty}(\Omega)} \leq\|e-\bar{e}\|_{E} .
$$

Using these feasible approximations, by Proposition 4.1 and (2.31) $-(2.32)$ we get

$$
\begin{aligned}
& \left(\mathcal{J}_{u}^{\prime}\left(\bar{u}_{e}, e\right)-\mathcal{J}_{u}^{\prime}\left(\bar{u}_{\bar{e}}, \bar{e}\right)\right)\left(\bar{u}_{\bar{e}}-\bar{u}_{e}\right) \\
= & \mathcal{J}_{u}^{\prime}\left(\bar{u}_{e}, e\right)\left(\bar{u}_{\bar{e}}-u_{\bar{e}}+u_{\bar{e}}-\bar{u}_{e}\right)-\mathcal{J}_{u}^{\prime}\left(\bar{u}_{\bar{e}}, \bar{e}\right)\left(\bar{u}_{\bar{e}}-u_{e}+u_{e}-\bar{u}_{e}\right) \\
\geq & \mathcal{J}_{u}^{\prime}\left(\bar{u}_{e}, e\right)\left(\bar{u}_{\bar{e}}-u_{\bar{e}}\right)-\mathcal{J}_{u}^{\prime}\left(\bar{u}_{\bar{e}}, \bar{e}\right)\left(u_{e}-\bar{u}_{e}\right)+\frac{\kappa}{2}\left\|u_{e}-\bar{u}_{\bar{e}}\right\|_{L^{1}(\Omega)}^{1+\frac{1}{\alpha}}+\frac{1}{2} \mathcal{J}_{u}^{\prime}\left(\bar{u}_{\bar{e}}, \bar{e}\right)\left(u_{e}-\bar{u}_{\bar{e}}\right) .
\end{aligned}
$$

We can rewrite

$$
\begin{aligned}
& \mathcal{J}_{u}^{\prime}\left(\bar{u}_{e}, e\right)\left(\bar{u}_{\bar{e}}-u_{\bar{e}}\right)-\mathcal{J}_{u}^{\prime}\left(\bar{u}_{\bar{e}}, \bar{e}\right)\left(u_{e}-\bar{u}_{e}\right) \\
= & \left(\mathcal{J}_{u}^{\prime}\left(\bar{u}_{e}, e\right)-\mathcal{J}_{u}^{\prime}\left(\bar{u}_{\bar{e}}, \bar{e}\right)\right) d+\mathcal{J}_{u}^{\prime}\left(\bar{u}_{e}, e\right)\left(\chi_{\Omega \backslash \Omega_{\sigma}}\left(\bar{u}_{\bar{e}}-u_{\bar{e}}\right)\right)-\mathcal{J}_{u}^{\prime}\left(\bar{u}_{\bar{e}}, \bar{e}\right)\left(\chi_{\Omega \backslash \Omega_{\sigma}}\left(u_{e}-\bar{u}_{e}\right)\right),
\end{aligned}
$$

where

$$
d:=\chi_{\Omega_{\sigma}}\left(\bar{u}_{\bar{e}}-u_{\bar{e}}\right)=\chi_{\Omega_{\sigma}}\left(u_{e}-\bar{u}_{e}\right)
$$

satisfying $\|d\|_{L^{\infty}(\Omega)} \leq\|e-\bar{e}\|_{E}$. Due to Tchebyshev's inequality, the measure of $\Omega \backslash \Omega_{\sigma}$ is bounded by $2 \sigma^{-1}\left\|\bar{u}_{e}-\bar{u}_{\bar{e}}\right\|_{L^{1}(\Omega)}$. Then we can estimate with $c>0$ independent of $e$ as follows

$$
\begin{aligned}
\left|\mathcal{J}_{u}^{\prime}\left(\bar{u}_{e}, e\right)\left(\bar{u}_{\bar{e}}-u_{\bar{e}}\right)-\mathcal{J}_{u}^{\prime}\left(\bar{u}_{\bar{e}}, \bar{e}\right)\left(u_{e}-\bar{u}_{e}\right)\right| & \\
\leq & |\Omega| \cdot\left\|\varphi_{\bar{u}_{e}, e}-\varphi_{\bar{u}_{\bar{e}}, \bar{e}}\right\|_{L^{\infty}(\Omega)}\|e-\bar{e}\|_{E} \\
& \quad+\left(\left\|\varphi_{\bar{u}_{e}, e}-\varphi_{\bar{u}_{\bar{e}}, \bar{e}}\right\|_{L^{\infty}(\Omega)}+2\left\|\varphi_{\bar{u}_{\bar{e}}, \bar{e}}\right\|_{L^{\infty}(\Omega)}\right) 2 \sigma^{-1}\left\|\bar{u}_{e}-\bar{u}_{\bar{e}}\right\|_{L^{1}(\Omega)}\|e-\bar{e}\|_{E} \\
& \leq c\left(\left\|\varphi_{\bar{u}_{e}, e}-\varphi_{\bar{u}_{\bar{e}}, \bar{e}}\right\|_{L^{\infty}(\Omega)}+\left(1+\left\|\varphi_{\bar{u}_{e}, e}-\varphi_{\bar{u}_{\bar{e}}, \bar{e}}\right\|_{L^{\infty}(\Omega)}\right)\left\|\bar{u}_{e}-\bar{u}_{\bar{e}}\right\|_{L^{1}(\Omega)}\right)\|e-\bar{e}\|_{E} .
\end{aligned}
$$

Since $\left\|\bar{u}_{e}-\bar{u}_{\bar{e}}\right\|_{L^{1}(\Omega)}$ is uniformly bounded with respect to $e$ due to the presence of the control constraints, we can simplify this inequality to

$$
\begin{aligned}
& \left|\mathcal{J}_{u}^{\prime}\left(\bar{u}_{e}, e\right)\left(\bar{u}_{e}-u_{e}\right)-\mathcal{J}_{u}^{\prime}\left(\bar{u}_{\bar{e}}, \bar{e}\right)\left(\bar{u}_{e}-u_{\bar{e}}\right)\right| \\
& \quad \leq c\left(\left\|\varphi_{\bar{u}_{e}, e}-\varphi_{\bar{u}_{\bar{e}}, \bar{e}}\right\|_{L^{\infty}(\Omega)}+\left\|\bar{u}_{e}-\bar{u}_{\bar{e}}\right\|_{L^{1}(\Omega)}\right)\|e-\bar{e}\|_{E} .
\end{aligned}
$$

It remains to develop a lower bound of $\left\|u_{e}-\bar{u}_{\bar{e}}\right\|_{L^{1}(\Omega)}^{1+\frac{1}{x}}$. By construction of $u_{e}$, we have

$$
\left\|\bar{u}_{e}-\bar{u}_{\bar{e}}\right\|_{L^{1}(\Omega)} \leq\left\|u_{e}-\bar{u}_{\bar{e}}\right\|_{L^{1}(\Omega)}+|\Omega| \cdot\|e-\bar{e}\|_{E} .
$$


This implies

$$
\left\|\bar{u}_{e}-\bar{u}_{\bar{e}}\right\|_{L^{1}(\Omega)}^{1+\frac{1}{\infty}} \leq 2^{\frac{1}{\infty}}\left(\left\|u_{e}-\bar{u}_{\bar{e}}\right\|_{L^{1}(\Omega)}^{1+\frac{1}{\infty}}+|\Omega|^{1+\frac{1}{\infty}}\|e-\bar{e}\|_{E}^{1+\frac{1}{\infty}}\right) .
$$

Collecting the inequalities (4.14)-(4.16) yields

$$
\begin{aligned}
& \left(\mathcal{J}_{u}^{\prime}\left(\bar{u}_{e}, e\right)-\mathcal{J}_{u}^{\prime}\left(\bar{u}_{\bar{e}}, \bar{e}\right)\right)\left(\bar{u}_{\bar{e}}-\bar{u}_{e}\right) \\
& \quad \geq \kappa^{\prime}\left\|\bar{u}_{e}-\bar{u}_{\bar{e}}\right\|_{L^{1}(\Omega)}^{1+\frac{1}{\infty}}+\frac{1}{2} \mathcal{J}_{u}^{\prime}\left(\bar{u}_{\bar{e}}, \bar{e}\right)\left(u_{e}-\bar{u}_{\bar{e}}\right) \\
& \quad-c\left(\|e-\bar{e}\|_{E}^{\frac{1}{\infty}}+\left\|\varphi_{\bar{u}_{e}, e}-\varphi_{\bar{u}_{\bar{e}}, \bar{e}}\right\|_{L^{\infty}(\Omega)}+\left\|\bar{u}_{e}-\bar{u}_{\bar{e}}\right\|_{L^{1}(\Omega)}\right)\|e-\bar{e}\|_{E}
\end{aligned}
$$

with $\kappa^{\prime}:=\kappa 2^{1-\frac{1}{\infty}}$ and $c$ independent of $e, \bar{u}_{e}$.

Theorem 4.14. Let $\bar{u}_{\bar{e}}$ be a strict bang-bang solution of problem (4.1) for $\bar{e} \in E$ and assume that (A1)-(A4) hold. Assume that the second-order condition (4.7) holds at $\bar{u}_{\bar{e}}$. Then, there exist $\eta>0$ and $c>0$ such that

$$
\left\|\bar{u}_{e}-\bar{u}_{\bar{e}}\right\|_{L^{1}(\Omega)} \leq c\|e-\bar{e}\|_{E}^{\min \{x, 1\}}
$$

for all $e \in B_{\eta}(\bar{e})$ and for any $\bar{u}_{e} \in \mathcal{U}_{a d}(e) \cap B_{\eta}^{p_{0}}\left(\bar{u}_{\bar{e}}\right)$ satisfying the first-order optimality system $(2.29)-(2.31)$.

Proof. By Lemma 4.13, we have

$$
\begin{aligned}
& \left(\mathcal{J}_{u}^{\prime}\left(\bar{u}_{e}, e\right)-\mathcal{J}_{u}^{\prime}\left(\bar{u}_{\bar{e}}, \bar{e}\right)\right)\left(\bar{u}_{\bar{e}}-\bar{u}_{e}\right) \\
& \quad \geq \kappa^{\prime}\left\|\bar{u}_{e}-\bar{u}_{\bar{e}}\right\|_{L^{1}(\Omega)}^{1+\frac{1}{x}}+\frac{1}{2} \mathcal{J}_{u}^{\prime}\left(\bar{u}_{\bar{e}}, \bar{e}\right)\left(u_{e}-\bar{u}_{\bar{e}}\right) \\
& \quad-c\left(\|e-\bar{e}\|_{E}^{\frac{1}{\infty}}+\left\|\varphi_{\bar{u}_{e}, e}-\varphi_{\bar{u}_{\bar{e}}, \bar{e}}\right\|_{L^{\infty}(\Omega)}+\left\|\bar{u}_{e}-\bar{u}_{\bar{e}}\right\|_{L^{1}(\Omega)}\right)\|e-\bar{e}\|_{E}
\end{aligned}
$$

with $u_{e} \in \mathcal{U}_{a d}(\bar{e})$ and $\left\|\bar{u}_{e}-u_{e}\right\|_{L^{\infty}(\Omega)} \leq\|e-\bar{e}\|_{E}$ as above. We write

$$
\begin{aligned}
& \left(\mathcal{J}_{u}^{\prime}\left(\bar{u}_{e}, e\right)-\mathcal{J}_{u}^{\prime}\left(\bar{u}_{\bar{e}}, \bar{e}\right)\right)\left(\bar{u}_{\bar{e}}-\bar{u}_{e}\right) \\
= & \left(\mathcal{J}_{u}^{\prime}\left(\bar{u}_{e}, e\right)-\mathcal{J}_{u}^{\prime}\left(\bar{u}_{e}, \bar{e}\right)\right)\left(\bar{u}_{\bar{e}}-\bar{u}_{e}\right)+\left(\mathcal{J}_{u}^{\prime}\left(\bar{u}_{e}, \bar{e}\right)-\mathcal{J}_{u}^{\prime}\left(\bar{u}_{\bar{e}}, \bar{e}\right)\right)\left(\bar{u}_{\bar{e}}-\bar{u}_{e}\right) .
\end{aligned}
$$

Using the representation (2.32) of $\mathcal{J}_{u}^{\prime}$ by adjoint states and the estimate of Lemma 4.11, we obtain

$$
\begin{aligned}
\left|\left(\mathcal{J}_{u}^{\prime}\left(\bar{u}_{e}, e\right)-\mathcal{J}_{u}^{\prime}\left(\bar{u}_{e}, \bar{e}\right)\right)\left(\bar{u}_{\bar{e}}-\bar{u}_{e}\right)\right| & \leq\left\|\mathcal{J}_{u}^{\prime}\left(\bar{u}_{e}, e\right)-\mathcal{J}_{u}^{\prime}\left(\bar{u}_{e}, \bar{e}\right)\right\|_{L^{\infty}(\Omega)}\left\|\bar{u}_{\bar{e}}-\bar{u}_{e}\right\|_{L^{1}(\Omega)} \\
& =\left\|\varphi_{\bar{u}_{e}, e}-\varphi_{\bar{u}_{e}, \bar{e}}\right\|_{L^{\infty}(\Omega)}\left\|\bar{u}_{\bar{e}}-\bar{u}_{e}\right\|_{L^{1}(\Omega)} \\
& \leq c\|e-\bar{e}\|_{E}\left\|\bar{u}_{\bar{e}}-\bar{u}_{e}\right\|_{L^{1}(\Omega)} .
\end{aligned}
$$

In addition, by Lemmas 4.10 and 4.11 we get

$$
\left\|\varphi_{\bar{u}_{e}, e}-\varphi_{\bar{u}_{\bar{e}}, \bar{e}}\right\|_{L^{\infty}(\Omega)} \leq c\left(\|e-\bar{e}\|_{E}+\left\|\bar{u}_{e}-\bar{u}_{\bar{e}}\right\|_{L^{1}(\Omega)}\right) .
$$

This shows

$$
\begin{gathered}
\kappa^{\prime}\left\|\bar{u}_{e}-\bar{u}_{\bar{e}}\right\|_{L^{1}(\Omega)}^{1+\frac{1}{x}}+\left(\mathcal{J}_{u}^{\prime}\left(\bar{u}_{e}, \bar{e}\right)-\mathcal{J}_{u}^{\prime}\left(\bar{u}_{\bar{e}}, \bar{e}\right)\right)\left(\bar{u}_{e}-\bar{u}_{\bar{e}}\right)+\frac{1}{2} \mathcal{J}_{u}^{\prime}\left(\bar{u}_{\bar{e}}, \bar{e}\right)\left(u_{e}-\bar{u}_{\bar{e}}\right) \\
\leq c\left(\|e-\bar{e}\|_{E}^{\frac{1}{\alpha}}+\|e-\bar{e}\|_{E}+\left\|\bar{u}_{e}-\bar{u}_{\bar{e}}\right\|_{L^{1}(\Omega)}\right)\|e-\bar{e}\|_{E} .
\end{gathered}
$$

By Taylor expansion, we find

$$
\left(\mathcal{J}_{u}^{\prime}\left(\bar{u}_{e}, \bar{e}\right)-\mathcal{J}_{u}^{\prime}\left(\bar{u}_{\bar{e}}, \bar{e}\right)\right)\left(\bar{u}_{e}-\bar{u}_{\bar{e}}\right)=\mathcal{J}_{u}^{\prime \prime}(\widehat{u}, \bar{e})\left(\bar{u}_{e}-\bar{u}_{\bar{e}}\right)^{2},
$$


where $\widehat{u}=\bar{u}_{\bar{e}}+\theta\left(\bar{u}_{e}-\bar{u}_{\bar{e}}\right)$ and $\theta \in(0,1)$. Let us define

$$
\Omega_{\tau}:=\left\{x \in \Omega:\left|\varphi_{\bar{u}_{\bar{e}}}\right| \leq \tau\right\}
$$

We now define

$$
v=\chi_{\Omega_{\tau}}\left(u_{e}-\bar{u}_{\bar{e}}\right), \quad w=\chi_{\Omega \backslash \Omega_{\tau}}\left(u_{e}-\bar{u}_{\bar{e}}\right), \quad \widetilde{w}:=\bar{u}_{e}-u_{e},
$$

such that $v+w+\widetilde{w}=\bar{u}_{e}-\bar{u}_{\bar{e}}$ and $v \in C_{\bar{u}_{\bar{e}}, p_{0}}^{\tau}$, for the definition of $C_{\bar{u}_{\bar{e}}, p_{0}}^{\tau}$ see (4.6). Moreover, we have $\|\widetilde{w}\|_{L^{\infty}(\Omega)} \leq\|e-\bar{e}\|_{E}$. Due to the feasibility $u_{e} \in \mathcal{U}_{a d}(\bar{e})$, we have

$$
\mathcal{J}_{u}^{\prime}\left(\bar{u}_{\bar{e}}, \bar{e}\right)\left(u_{e}-\bar{u}_{\bar{e}}\right)=\int_{\Omega}\left|\varphi_{\bar{u}_{\bar{e}}}\right| \cdot\left|u_{e}-\bar{u}_{\bar{e}}\right| d x \geq \tau\|w\|_{L^{1}(\Omega)} .
$$

From the definition of $v$ and $w$ we get

$$
\begin{aligned}
\mathcal{J}_{u}^{\prime \prime}(\widehat{u}, \bar{e}) & \left(\bar{u}_{e}-\bar{u}_{\bar{e}}\right)^{2}+\frac{1}{2} \mathcal{J}_{u}^{\prime}\left(\bar{u}_{\bar{e}}, \bar{e}\right)\left(u_{e}-\bar{u}_{\bar{e}}\right) \\
\geq & \frac{\tau}{2}\|w\|_{L^{1}(\Omega)}+\mathcal{J}_{u}^{\prime \prime}\left(\bar{u}_{\bar{e}}, \bar{e}\right) v^{2}+\left(\mathcal{J}_{u}^{\prime \prime}(\widehat{u}, \bar{e})-\mathcal{J}_{u}^{\prime \prime}\left(\bar{u}_{\bar{e}}, \bar{e}\right)\right) v^{2} \\
& \quad+\mathcal{J}_{u}^{\prime \prime}(\widehat{u}, \bar{e})(w+\widetilde{w})^{2}+2 \mathcal{J}_{u}^{\prime \prime}(\widehat{u}, \bar{e})(v, w+\widetilde{w})
\end{aligned}
$$

Let us set for abbreviation $z_{v}:=z_{\bar{u}_{\bar{e}}, v}^{\bar{e}}, z_{\widehat{u}, v}:=z_{\widehat{u}, v}^{\bar{e}}$, and similarly for $z_{\widehat{u}, w}, z_{\widehat{u}, \widetilde{w}}$. Using the second-order condition (4.7), the continuity estimate of $\mathcal{J}_{u}^{\prime \prime}$ of Lemma 4.8, and the estimate of $\mathcal{J}_{u}^{\prime \prime}$ of Lemma 4.9, we get

$$
\begin{aligned}
\mathcal{J}_{u}^{\prime \prime}(\widehat{u}, \bar{e})\left(\bar{u}_{e}-\bar{u}_{\bar{e}}\right)^{2}+\frac{1}{2} \mathcal{J}_{u}^{\prime}\left(\bar{u}_{\bar{e}}, \bar{e}\right)\left(u_{e}-\bar{u}_{\bar{e}}\right) \\
\geq \frac{\tau}{2}\|w\|_{L^{1}(\Omega)}+\delta\left\|z_{v}\right\|_{L^{2}(\Omega)}^{2}-\frac{\delta}{4}\left\|z_{v}\right\|_{L^{2}(\Omega)}^{2}-2 K_{M}\left(\left\|z_{\widehat{u}, w}\right\|_{L^{2}(\Omega)}^{2}+\left\|z_{\widehat{u}, \widetilde{w}}\right\|_{L^{2}(\Omega)}^{2}\right) \\
\quad-2 K_{M}\left(\left\|z_{v}\right\|_{L^{2}(\Omega)}+\left\|z_{v}-z_{\widehat{u}, v}\right\|_{L^{2}(\Omega)}\right)\left(\left\|z_{\widehat{u}, w}\right\|_{L^{2}(\Omega)}+\left\|z_{\widehat{u}, \widetilde{w}}\right\|_{L^{2}(\Omega)}\right)
\end{aligned}
$$

for all $\bar{u}_{e}$ in some ball $B_{\eta}^{p_{0}}\left(\bar{u}_{\bar{e}}\right)$ with $\eta>0$. Using Lemma 4.12, we estimate

$$
\left\|z_{\widehat{u}, w}\right\|_{L^{2}(\Omega)} \leq c\|w\|_{L^{1}(\Omega)}
$$

and

$$
\left\|z_{\widehat{u}, \widetilde{w}}\right\|_{L^{2}(\Omega)} \leq c\|\widetilde{w}\|_{L^{\infty}(\Omega)} \leq c\|e-\bar{e}\|_{E} .
$$

Applying Lemma 4.12, we find

$$
\left\|z_{v}-z_{\widehat{u}, v}\right\|_{L^{2}(\Omega)} \leq c \eta\left\|z_{v}\right\|_{L^{2}(\Omega)} .
$$

Using this estimate in (4.19), we obtain

$$
\begin{aligned}
\mathcal{J}_{u}^{\prime \prime}(\widehat{u}, \bar{e}) & \left(\bar{u}_{e}-\bar{u}_{\bar{e}}\right)^{2}+\frac{1}{2} \mathcal{J}_{u}^{\prime}\left(\bar{u}_{\bar{e}}, \bar{e}\right)\left(u_{e}-\bar{u}_{\bar{e}}\right) \\
\geq & \frac{\tau}{2}\|w\|_{L^{1}(\Omega)}+\frac{3}{4} \delta\left\|z_{v}\right\|_{L^{2}(\Omega)}^{2} \\
& \quad-c\left(\|w\|_{L^{1}(\Omega)}^{2}+\|e-\bar{e}\|_{E}^{2}+\left\|z_{v}\right\|_{L^{2}(\Omega)}\left(\|w\|_{L^{1}(\Omega)}+\|e-\bar{e}\|_{E}\right)\right)
\end{aligned}
$$

with some $c>0$ independent of $e$ and $\bar{u}_{e}$. Using Young's inequality, the following inequality can be derived:

$$
\begin{aligned}
\mathcal{J}_{u}^{\prime \prime}(\widehat{u}, \bar{e})\left(\bar{u}_{e}-\bar{u}_{\bar{e}}\right)^{2}+\frac{1}{2} \mathcal{J}_{u}^{\prime}\left(\bar{u}_{\bar{e}}, \bar{e}\right)\left(u_{e}-\bar{u}_{\bar{e}}\right) \\
\quad \geq\|w\|_{L^{1}(\Omega)}\left(\frac{\tau}{2}-c_{1}\|w\|_{L^{1}(\Omega)}\right)+\frac{1}{2} \delta\left\|z_{v}\right\|_{L^{2}(\Omega)}^{2}-c_{2}\|e-\bar{e}\|_{E}^{2}
\end{aligned}
$$


By making $\eta$ smaller if necessary, we can achieve

$$
\|w\|_{L^{1}(\Omega)}\left(\frac{\tau}{2}-c_{1}\|w\|_{L^{1}(\Omega)}\right) \geq \frac{\tau}{4}\|w\|_{L^{1}(\Omega)} .
$$

This shows

$$
\mathcal{J}_{u}^{\prime \prime}(\widehat{u}, \bar{e})\left(\bar{u}_{e}-\bar{u}_{\bar{e}}\right)^{2}+\frac{1}{2} \mathcal{J}_{u}^{\prime}\left(\bar{u}_{\bar{e}}, \bar{e}\right)\left(u_{e}-\bar{u}_{\bar{e}}\right) \geq \frac{\tau}{4}\|w\|_{L^{1}(\Omega)}+\frac{\delta}{2}\left\|z_{v}\right\|_{L^{2}(\Omega)}^{2}-C\|e-\bar{e}\|_{E}^{2} .
$$

Together with (4.18), this implies

$$
\begin{aligned}
& \frac{\tau}{4}\|w\|_{L^{1}(\Omega)}+\frac{\delta}{2}\left\|z_{v}\right\|_{L^{2}(\Omega)}^{2}+\kappa^{\prime}\left\|\bar{u}_{e}-\bar{u}_{\bar{e}}\right\|_{L^{1}(\Omega)}^{1+\frac{1}{\infty}} \\
& \quad \leq c\left(\|e-\bar{e}\|_{E}^{\frac{1}{\infty}}+\|e-\bar{e}\|_{E}+\left\|\bar{u}_{e}-\bar{u}_{\bar{e}}\right\|_{L^{1}(\Omega)}\right)\|e-\bar{e}\|_{E}
\end{aligned}
$$

With Young's inequality we obtain

$$
c\left\|\bar{u}_{e}-\bar{u}_{\bar{e}}\right\|_{L^{1}(\Omega)}\|e-\bar{e}\|_{E} \leq \frac{\kappa^{\prime}}{2}\left\|\bar{u}_{e}-\bar{u}_{\bar{e}}\right\|_{L^{1}(\Omega)}^{1+\frac{1}{\infty}}+c\|e-\bar{e}\|_{E}^{\Re+1} .
$$

Thus, we arrive at the inequality

$$
\frac{\tau}{4}\|w\|_{L^{1}(\Omega)}+\frac{\delta}{2}\left\|z_{v}\right\|_{L^{2}(\Omega)}^{2}+\kappa^{\prime}\left\|\bar{u}_{e}-\bar{u}_{\bar{e}}\right\|_{L^{1}(\Omega)}^{1+\frac{1}{æ}} \leq c\left(\|e-\bar{e}\|_{E}^{1+\frac{1}{æ}}+\|e-\bar{e}\|_{E}^{2}+\|e-\bar{e}\|_{E}^{æ+1}\right) .
$$

For $æ \in[0,1], æ+1$ is the smallest exponent on the right-hand side, if $æ>1$, then $1+\frac{1}{æ}$ is the smallest exponent. This proves

$$
\left\|\bar{u}_{e}-\bar{u}_{\bar{e}}\right\|_{L^{1}(\Omega)} \leq C\|e-\bar{e}\|_{E}^{\min \{æ, 1\}},
$$

and therefore we obtain (4.17).

The following theorem shows that the (global) solution map $S: \operatorname{dom} \mathcal{U}_{a d} \rightrightarrows L^{1}(\Omega)$ admits a local upper Hölderian selection at a given point $\left(\bar{e}, \bar{u}_{\bar{e}}\right) \in \operatorname{gph} S$ provided that for every $e \in \operatorname{dom} \mathcal{U}_{a d}$ near $\bar{e}$, problem (4.1) has a (global) solution $\bar{u}_{e}$ near $\bar{u}_{\bar{e}}$.

Theorem 4.15. Assume that all the assumptions of Theorem 4.14 are satisfied and let $\left(\bar{e}, \bar{u}_{\bar{e}}\right) \in \operatorname{gph} S$ be such that $\bar{u}_{\bar{e}}$ is strict in a neighborhood $\bar{B}_{\varepsilon}^{p_{0}}\left(\bar{u}_{\bar{e}}\right)$ with $\varepsilon>0$. Assume further that for every $e \in \operatorname{dom} \mathcal{U}_{a d}$ near $\bar{e}$, problem (4.1) has a solution $\bar{u}_{e}$ satisfying $\bar{u}_{e} \in \bar{B}_{\varepsilon}^{p_{0}}\left(\bar{u}_{\bar{e}}\right)$. Then, the solution map $S: \operatorname{dom} \mathcal{U}_{a d} \rightrightarrows L^{1}(\Omega)$ admits a local upper Hölderian selection with the exponent $\rightsquigarrow[0,1]$ at the point $\left(\bar{e}, \bar{u}_{\bar{e}}\right)$.

Proof. According to Theorems 4.5 and 4.14, there exist constants $\eta>0$ and $c>0$ satisfying

$$
\left\|\bar{u}_{e}-\bar{u}_{\bar{e}}\right\|_{L^{1}(\Omega)} \leq c\|e-\bar{e}\|_{E}^{\min \{æ, 1\}}, \forall e \in \bar{B}_{\eta}(\bar{e}),
$$

where $\bar{u}_{e}$ is a solution of problem (4.1) with respect to $e \in E$ satisfying $\bar{u}_{e} \in \bar{B}_{\varepsilon}^{p_{0}}\left(\bar{u}_{\bar{e}}\right)$. Define a single-valued function $h: \operatorname{dom} \mathcal{G} \rightarrow L^{1}(\Omega)$ by $h(\bar{e})=\bar{u}_{\bar{e}}$ and $h(e)=\bar{u}_{e}$ for $e \in \operatorname{dom} \mathcal{G}$. Then, for $e \in \bar{B}_{\eta}(\bar{e}) \cap \operatorname{dom} \mathcal{G}$, by (4.20) we obtain

$$
\|h(e)-h(\bar{e})\|_{L^{1}(\Omega)} \leq c\|e-\bar{e}\|_{E}^{æ^{\prime}},
$$

which yields that $h$ is a local upper Hölderian selection of $S(\cdot)$ at the point $\left(\bar{e}, \bar{u}_{\bar{e}}\right)$, where the exponent $æ^{\prime}=\min \{æ, 1\} \in[0,1]$.

Corollary 4.16. Assume that all the assumptions of Theorem 4.14 are satisfied and let $\left(\bar{e}, \bar{u}_{\bar{e}}\right) \in \operatorname{gph} S$ be such that $S(\bar{e})=\left\{\bar{u}_{\bar{e}}\right\}$. Then, the solution map $S: \operatorname{dom} \mathcal{U}_{a d} \rightrightarrows L^{1}(\Omega)$ of problem (4.1) has a local upper Hölderian selection at $\left(\bar{e}, \bar{u}_{\bar{e}}\right)$ with the exponent $æ \in[0,1]$.

Proof. By applying Theorem 4.14 and Corollary 4.6 and arguing similarly as in the proof of Theorem 4.15, we obtain the assertion of the corollary. 


\subsection{Lower estimate for regular subdifferential of $\mu(\cdot)$}

In this subsection, we will establish a characterization of regular subgradients of the marginal function $\mu(\cdot)$ in a subspace $E_{1}^{*}$ of $E^{*}$, where $E_{1}^{*}$ is defined as follows

$$
\begin{aligned}
E_{1}^{*}: & =L^{2}(\Omega) \times L^{2}(\Omega) \times L^{1}(\Omega) \times L^{1}(\Omega) \\
& \subset L^{2}(\Omega) \times L^{2}(\Omega) \times L^{\infty}(\Omega)^{*} \times L^{\infty}(\Omega)^{*}=E^{*} .
\end{aligned}
$$

We now define the set

$$
\begin{aligned}
\widehat{\Xi}\left(\left(\bar{e}, \bar{u}_{\bar{e}}\right) ; g p h \mathcal{U}_{a d}\right)=\{ & \left(e^{*}, u^{*}\right) \in E_{1}^{*} \times L^{2}(\Omega) \mid e^{*}=\left(0,0, e_{\alpha}^{*}, e_{\beta}^{*}\right), u^{*}=-e_{\alpha}^{*}-e_{\beta}^{*}, \\
& \left.e_{\alpha}^{*}\right|_{\Omega_{1}\left(\bar{e}, \bar{u}_{\bar{e}}\right)} \geq 0,\left.e_{\alpha}^{*}\right|_{\Omega \backslash \Omega_{1}\left(\bar{e}, \bar{u}_{\bar{e}}\right)}=0, \\
& \left.\left.e_{\beta}^{*}\right|_{\Omega_{3}\left(\bar{e}, \bar{u}_{\bar{e}}\right)} \leq 0,\left.e_{\beta}^{*}\right|_{\Omega \backslash \Omega_{3}\left(\bar{e}, \bar{u}_{\bar{e}}\right)}=0\right\} .
\end{aligned}
$$

By arguing similarly as the proof of Lemma 3.2 we get

$$
\widehat{\Xi}\left(\left(\bar{e}, \bar{u}_{\bar{e}}\right) ; \operatorname{gph} \mathcal{U}_{a d}\right) \subset \widehat{N}\left(\left(\bar{e}, \bar{u}_{\bar{e}}\right) ; \operatorname{gph} \mathcal{U}_{a d}\right) .
$$

Consequently, by setting

$$
\begin{aligned}
\widehat{\Lambda}^{*} \mathcal{G}\left(\bar{e}, \bar{u}_{\bar{e}}\right)\left(u^{*}\right)= & \left\{e^{*} \in E_{1}^{*} \mid\left(e^{*},-u^{*}\right) \in \widehat{\Xi}\left(\left(\bar{e}, \bar{u}_{\bar{e}}\right) ; \operatorname{gph} \mathcal{G}\right)\right\} \\
= & \left\{e^{*} \in E_{1}^{*} \mid e^{*}=\left(0,0, e_{\alpha}^{*}, e_{\beta}^{*}\right), u^{*}=e_{\alpha}^{*}+e_{\beta}^{*},\right. \\
& \left.e_{\alpha}^{*}\right|_{\Omega_{1}\left(\bar{e}, \bar{u}_{\bar{e}}\right)} \geq 0,\left.e_{\alpha}^{*}\right|_{\Omega \backslash \Omega_{1}\left(\bar{e}, \bar{u}_{\bar{e}}\right)}=0 \\
& \left.\left.e_{\beta}^{*}\right|_{\Omega_{3}\left(\bar{e}, \bar{u}_{\bar{e}}\right)} \leq 0,\left.e_{\beta}^{*}\right|_{\Omega \backslash \Omega_{3}\left(\bar{e}, \bar{u}_{\bar{e}}\right)}=0\right\}
\end{aligned}
$$

we deduce that

$$
\widehat{\Lambda}^{*} \mathcal{G}\left(\bar{e}, \bar{u}_{\bar{e}}\right)\left(u^{*}\right) \subset \widehat{D}^{*} \mathcal{G}\left(\bar{e}, \bar{u}_{\bar{e}}\right)\left(u^{*}\right) .
$$

Motivated by the estimate (4.22), we are going to establish a lower estimate for $\widehat{\partial} \mu(\bar{e})$ via a characterization of regular subgradients of the marginal function $\mu(\cdot)$ in the subspace $E_{1}^{*}$ of $E^{*}$ in the forthcoming theorem.

Theorem 4.17. Assume that (A1)-(A3) hold and let $\left(\bar{e}, \bar{u}_{\bar{e}}\right) \in \operatorname{gph} S$ be given. Then, for any $\widehat{e}^{*}=\left(\widehat{e}_{y}^{*}, \widehat{e}_{J}^{*}, \widehat{e}_{\alpha}^{*}, \widehat{e}_{\beta}^{*}\right) \in \widehat{\partial} \mu(\bar{e}) \cap E_{1}^{*}$, the following holds

$$
\left\{\begin{array}{l}
\widehat{e}_{y}^{*}=\varphi_{\bar{u}_{\bar{e}}, \bar{e}} \\
\widehat{e}_{J}^{*}=y_{\bar{u}_{\bar{e}}+\bar{e}_{y}} \\
\left.\widehat{e}_{\alpha}^{*}\right|_{\Omega_{1}\left(\bar{e}, \bar{u}_{\bar{e}}\right)} \geq 0,\left.\widehat{e}_{\alpha}^{*}\right|_{\Omega \backslash \Omega_{1}\left(\bar{e}, \bar{u}_{\bar{e}}\right)}=0 \\
\left.\widehat{e}_{\beta}^{*}\right|_{\Omega_{3}\left(\bar{e}, \bar{u}_{\bar{e}}\right)} \leq 0,\left.\widehat{e}_{\beta}^{*}\right|_{\Omega \backslash \Omega_{3}\left(\bar{e}, \bar{u}_{\bar{e}}\right)}=0 \\
\widehat{e}_{\alpha}^{*}+\widehat{e}_{\beta}^{*}=\varphi_{\bar{u}_{\bar{e}}, \bar{e}}
\end{array}\right.
$$

In addition, assume that the solution map $S(\cdot)$ admits a local upper Hölderian selection $h(\cdot)$ with $h(\bar{e})=\bar{u}_{\bar{e}}, h(e)=\bar{u}_{e}$, and

$$
\|h(e)-h(\bar{e})\|_{L^{1}(\Omega)} \leq c\|e-\bar{e}\|_{E}^{\infty}, \forall e \in \bar{B}_{\eta}(\bar{e}) \cap \operatorname{dom} \mathcal{G},
$$

for some $\eta>0, c>0$, and $\gg 1 / 2$. If an element $\widehat{e}^{*}=\left(\widehat{e}_{y}^{*}, \widehat{e}_{J}^{*}, \widehat{e}_{\alpha}^{*}, \widehat{e}_{\beta}^{*}\right) \in E_{1}^{*}$ satisfies (4.23), then $\widehat{e}^{*} \in \widehat{\partial} \mu(\bar{e})$. 
Proof. By definition, we have $\widehat{e}^{*}=\left(\widehat{e}_{y}^{*}, \widehat{e}_{J}^{*}, \widehat{e}_{\alpha}^{*}, \widehat{e}_{\beta}^{*}\right) \in \widehat{\partial} \mu(\bar{e})$ if and only if

$$
\liminf _{e \rightarrow \bar{e}} \frac{\mu(e)-\mu(\bar{e})-\left\langle\widehat{e}^{*}, e-\bar{e}\right\rangle}{\|e-\bar{e}\|_{E}} \geq 0
$$

Suppose that $\widehat{e}^{*}=\left(\widehat{e}_{y}^{*}, \widehat{e}_{J}^{*}, \widehat{e}_{\alpha}^{*}, \widehat{e}_{\beta}^{*}\right) \in \widehat{\partial} \mu(\bar{e}) \cap E_{1}^{*}$. We verify that $\widehat{e}^{*}=\left(\widehat{e}_{y}^{*}, \widehat{e}_{J}^{*}, \widehat{e}_{\alpha}^{*}, \widehat{e}_{\beta}^{*}\right)$ satisfies (4.23). By the inclusion $\widehat{e}^{*} \in \widehat{\partial} \mu(\bar{e})$, we have (4.25).

Let $e=\left(\bar{e}_{y}, \bar{e}_{J}, e_{\alpha}, e_{\beta}\right) \rightarrow\left(\bar{e}_{y}, \bar{e}_{J}, \bar{e}_{\alpha}, \bar{e}_{\beta}\right)=\bar{e}$ with $\left(e_{\alpha}, e_{\beta}\right)$ being chosen the same in the proof of Lemma 3.2. Then, we have $\bar{u}_{\bar{e}} \in \mathcal{G}(e)$, and thus $\mathcal{J}\left(\bar{u}_{\bar{e}}, e\right) \geq \mu(e)$. Since $\mathcal{J}(\cdot, \cdot)$ does not depend on $e_{\alpha}$ and $e_{\beta}$, we have $\mathcal{J}\left(\bar{u}_{\bar{e}}, \bar{e}\right)=\mathcal{J}\left(\bar{u}_{\bar{e}}, e\right)$ which yields $\mathcal{J}\left(\bar{u}_{\bar{e}}, \bar{e}\right) \geq \mu(e)$. Note that $\mu(\bar{e})=\mathcal{J}\left(\bar{u}_{\bar{e}}, \bar{e}\right)$ due to $\left(\bar{e}, \bar{u}_{\bar{e}}\right) \in \operatorname{gph} S$. Consequently, from (4.25) we obtain

$$
\liminf _{e \rightarrow \bar{e}} \frac{-\left\langle\widehat{e}_{\alpha}^{*}, e_{\alpha}-\bar{e}_{\alpha}\right\rangle-\left\langle\widehat{e}_{\beta}^{*}, e_{\beta}-\bar{e}_{\beta}\right\rangle}{\left\|e_{\alpha}-\bar{e}_{\alpha}\right\|_{L^{\infty}(\Omega)}+\left\|e_{\beta}-\bar{e}_{\beta}\right\|_{L^{\infty}(\Omega)}} \geq 0 .
$$

Using (4.26) and arguing similarly as the proof of Lemma 3.2 we deduce that

$$
\left\{\begin{array}{l}
\left.\widehat{e}_{\alpha}^{*}\right|_{\Omega_{1}\left(\bar{e}, \bar{u}_{\bar{e}}\right)} \geq 0,\left.\widehat{e}_{\alpha}^{*}\right|_{\Omega \backslash \Omega_{1}\left(\bar{e}, \bar{u}_{\bar{e}}\right)}=0 \\
\left.\widehat{e}_{\beta}^{*}\right|_{\Omega_{3}\left(\bar{e}, \bar{u}_{\bar{e}}\right)} \leq 0,\left.\widehat{e}_{\beta}^{*}\right|_{\Omega \backslash \Omega_{3}\left(\bar{e}, \bar{u}_{\bar{e}}\right)}=0
\end{array}\right.
$$

Let $e=\left(e_{y}, e_{J}, \bar{e}_{\alpha}, \bar{e}_{\beta}\right) \rightarrow\left(\bar{e}_{y}, \bar{e}_{J}, \bar{e}_{\alpha}, \bar{e}_{\beta}\right)=\bar{e}$. Then, $\mathcal{J}\left(\bar{u}_{\bar{e}}, e\right) \geq \mu(e)$ by $\bar{u}_{\bar{e}} \in \mathcal{G}(\bar{e})=\mathcal{G}(e)$. Combining this with (4.25) we obtain

$$
\liminf _{e \rightarrow \bar{e}} \frac{\mathcal{J}\left(\bar{u}_{\bar{e}}, e\right)-\mathcal{J}\left(\bar{u}_{\bar{e}}, \bar{e}\right)-\left\langle\left(\widehat{e}_{y}^{*}, \widehat{e}_{J}^{*}\right),\left(e_{y}, e_{J}\right)-\left(\bar{e}_{y}, \bar{e}_{J}\right)\right\rangle}{\left\|\left(e_{y}, e_{J}\right)-\left(\bar{e}_{y}, \bar{e}_{J}\right)\right\|_{L^{2}(\Omega) \times L^{2}(\Omega)}} \geq 0 .
$$

We have

$$
\begin{aligned}
& \frac{\mathcal{J}\left(\bar{u}_{\bar{e}}, e\right)-\mathcal{J}\left(\bar{u}_{\bar{e}}, \bar{e}\right)-\left\langle\left(\widehat{e}_{y}^{*}, \widehat{e}_{J}^{*}\right),\left(e_{y}, e_{J}\right)-\left(\bar{e}_{y}, \bar{e}_{J}\right)\right\rangle}{\left\|\left(e_{y}, e_{J}\right)-\left(\bar{e}_{y}, \bar{e}_{J}\right)\right\|_{L^{2}(\Omega) \times L^{2}(\Omega)}} \\
= & \frac{\mathcal{J}\left(\bar{u}_{\bar{e}}, e\right)-\mathcal{J}\left(\bar{u}_{\bar{e}}, \bar{e}\right)-\left\langle\left(\mathcal{J}_{e_{y}}^{\prime}\left(\bar{u}_{\bar{e}}, \bar{e}\right), \mathcal{J}_{e_{J}}^{\prime}\left(\bar{u}_{\bar{e}}, \bar{e}\right)\right),\left(e_{y}, e_{J}\right)-\left(\bar{e}_{y}, \bar{e}_{J}\right)\right\rangle}{\left\|\left(e_{y}, e_{J}\right)-\left(\bar{e}_{y}, \bar{e}_{J}\right)\right\|_{L^{2}(\Omega) \times L^{2}(\Omega)}} \\
& +\frac{\left\langle\left(\mathcal{J}_{e_{y}}^{\prime}\left(\bar{u}_{\bar{e}}, \bar{e}\right)-\widehat{e}_{y}^{*}, \mathcal{J}_{e_{J}}^{\prime}\left(\bar{u}_{\bar{e}}, \bar{e}\right)-\widehat{e}_{J}^{*}\right),\left(e_{y}, e_{J}\right)-\left(\bar{e}_{y}, \bar{e}_{J}\right)\right\rangle}{\left\|\left(e_{y}, e_{J}\right)-\left(\bar{e}_{y}, \bar{e}_{J}\right)\right\|_{L^{2}(\Omega) \times L^{2}(\Omega)}} .
\end{aligned}
$$

Since $\mathcal{J}(\cdot, \cdot)$ is Fréchet differentiable in $\left(e_{y}, e_{J}\right)$ at the point $\left(\bar{e}_{y}, \bar{e}_{J}\right)$, we have

$$
\lim _{\left(e_{y}, e_{J}\right) \rightarrow\left(\bar{e}_{y}, \bar{e}_{J}\right)} \frac{\mathcal{J}\left(\bar{u}_{\bar{e}}, e\right)-\mathcal{J}\left(\bar{u}_{\bar{e}}, \bar{e}\right)-\left\langle\left(\mathcal{J}_{e_{y}}^{\prime}\left(\bar{u}_{\bar{e}}, \bar{e}\right), \mathcal{J}_{e_{J}}^{\prime}\left(\bar{u}_{\bar{e}}, \bar{e}\right)\right),\left(e_{y}, e_{J}\right)-\left(\bar{e}_{y}, \bar{e}_{J}\right)\right\rangle}{\left\|\left(e_{y}, e_{J}\right)-\left(\bar{e}_{y}, \bar{e}_{J}\right)\right\|_{L^{2}(\Omega) \times L^{2}(\Omega)}}=0 .
$$

Hence, from (4.28) and (4.29) it follows that

$$
\liminf _{\left(e_{y}, e_{J}\right) \rightarrow\left(\bar{e}_{y}, \bar{e}_{J}\right)} \frac{\left\langle\left(\mathcal{J}_{e_{y}}^{\prime}\left(\bar{u}_{\bar{e}}, \bar{e}\right)-\widehat{e}_{y}^{*}, \mathcal{J}_{e_{J}}^{\prime}\left(\bar{u}_{\bar{e}}, \bar{e}\right)-\widehat{e}_{J}^{*}\right),\left(e_{y}, e_{J}\right)-\left(\bar{e}_{y}, \bar{e}_{J}\right)\right\rangle}{\left\|\left(e_{y}, e_{J}\right)-\left(\bar{e}_{y}, \bar{e}_{J}\right)\right\|_{L^{2}(\Omega) \times L^{2}(\Omega)}} \geq 0,
$$

which yields $\left(\mathcal{J}_{e_{y}}^{\prime}\left(\bar{u}_{\bar{e}}, \bar{e}\right)-\widehat{e}_{y}^{*}, \mathcal{J}_{e_{J}}^{\prime}\left(\bar{u}_{\bar{e}}, \bar{e}\right)-\widehat{e}_{J}^{*}\right)=\left(0_{L^{2}(\Omega)}, 0_{L^{2}(\Omega)}\right)$. Hence, we have

$$
\left\{\begin{array}{l}
\widehat{e}_{y}^{*}=\mathcal{J}_{e_{y}}^{\prime}\left(\bar{u}_{\bar{e}}, \bar{e}\right)=\mathcal{J}_{u}^{\prime}\left(\bar{u}_{\bar{e}}, \bar{e}\right)=\varphi_{\bar{u}_{\bar{e}}, \bar{e}} \\
\widehat{e}_{J}^{*}=\mathcal{J}_{e_{J}}^{\prime}\left(\bar{u}_{\bar{e}}, \bar{e}\right)=G\left(\bar{u}_{\bar{e}}+\bar{e}_{y}\right)=y_{\bar{u}_{\bar{e}}+\bar{e}_{y}} .
\end{array}\right.
$$


Let $e=\left(\bar{e}_{y}, \bar{e}_{J}, e_{\alpha}, e_{\beta}\right) \rightarrow\left(\bar{e}_{y}, \bar{e}_{J}, \bar{e}_{\alpha}, \bar{e}_{\beta}\right)=\bar{e}$ with $e_{\alpha}-\bar{e}_{\alpha}=e_{\beta}-\bar{e}_{\beta}$ and let $u \in \mathcal{G}(e)$ with $u-\bar{u}_{\bar{e}}=e_{\alpha}-\bar{e}_{\alpha}$. Note that $u \rightarrow \bar{u}_{\bar{e}}$ as $e \rightarrow \bar{e}$, and $\mathcal{J}(u, \bar{e})=\mathcal{J}(u, e) \geq \mu(e)$. From (4.25) it follows that

$$
\liminf _{e_{\alpha} \rightarrow \bar{e}_{\alpha}} \frac{\mathcal{J}(u, \bar{e})-\mathcal{J}\left(\bar{u}_{\bar{e}}, \bar{e}\right)-\left\langle\widehat{e}_{\alpha}^{*}+\widehat{e}_{\beta}^{*}, e_{\alpha}-\bar{e}_{\alpha}\right\rangle}{2\left\|e_{\alpha}-\bar{e}_{\alpha}\right\|_{L^{\infty}(\Omega)}} \geq 0 .
$$

By the choice of $e_{\alpha}, e_{\beta}$, and $u$ as above, we have

$$
\begin{aligned}
& \frac{\mathcal{J}(u, \bar{e})-\mathcal{J}\left(\bar{u}_{\bar{e}}, \bar{e}\right)-\left\langle\widehat{e}_{\alpha}^{*}+\widehat{e}_{\beta}^{*}, e_{\alpha}-\bar{e}_{\alpha}\right\rangle}{2\left\|e_{\alpha}-\bar{e}_{\alpha}\right\|_{L^{\infty}(\Omega)}} \\
= & \frac{\mathcal{J}(u, \bar{e})-\mathcal{J}\left(\bar{u}_{\bar{e}}, \bar{e}\right)-\left\langle\mathcal{J}_{u}^{\prime}\left(\bar{u}_{\bar{e}}, \bar{e}\right), u-\bar{u}_{\bar{e}}\right\rangle+\left\langle\mathcal{J}_{u}^{\prime}\left(\bar{u}_{\bar{e}}, \bar{e}\right)-\widehat{e}_{\alpha}^{*}-\widehat{e}_{\beta}^{*}, e_{\alpha}-\bar{e}_{\alpha}\right\rangle}{2\left\|e_{\alpha}-\bar{e}_{\alpha}\right\|_{L^{\infty}(\Omega)}} \\
= & \frac{o\left(\left\|u-\bar{u}_{\bar{e}}\right\|_{L^{2}(\Omega)}\right)+\left\langle\mathcal{J}_{u}^{\prime}\left(\bar{u}_{\bar{e}}, \bar{e}\right)-\widehat{e}_{\alpha}^{*}-\widehat{e}_{\beta}^{*}, e_{\alpha}-\bar{e}_{\alpha}\right\rangle}{2\left\|e_{\alpha}-\bar{e}_{\alpha}\right\|_{L^{\infty}(\Omega)}} .
\end{aligned}
$$

From this and (4.31) with noting that

$$
\lim _{e_{\alpha} \rightarrow \bar{e}_{\alpha}} \frac{o\left(\left\|u-\bar{u}_{\bar{e}}\right\|_{L^{2}(\Omega)}\right)}{2\left\|e_{\alpha}-\bar{e}_{\alpha}\right\|_{L^{\infty}(\Omega)}}=0
$$

we deduce

$$
\liminf _{e \rightarrow \bar{e}} \frac{\left\langle\mathcal{J}_{u}^{\prime}\left(\bar{u}_{\bar{e}}, \bar{e}\right)-\widehat{e}_{\alpha}^{*}-\widehat{e}_{\beta}^{*}, e_{\alpha}-\bar{e}_{\alpha}\right\rangle}{2\left\|e_{\alpha}-\bar{e}_{\alpha}\right\|_{L^{\infty}(\Omega)}} \geq 0,
$$

which yields $\mathcal{J}_{u}^{\prime}\left(\bar{u}_{\bar{e}}, \bar{e}\right)-\widehat{e}_{\alpha}^{*}-\widehat{e}_{\beta}^{*}=0_{L^{\infty}(\Omega)^{*}}$. Hence, we obtain

$$
\widehat{e}_{\alpha}^{*}+\widehat{e}_{\beta}^{*}=\mathcal{J}_{u}^{\prime}\left(\bar{u}_{\bar{e}}, \bar{e}\right)=\varphi_{\bar{u}_{\bar{e}}, \bar{e}} .
$$

Combining (4.27), (4.30) and (4.32) we obtain (4.23).

Conversely, we show that if $\widehat{e}^{*}=\left(\widehat{e}_{y}^{*}, \widehat{e}_{J}^{*}, \widehat{e}_{\alpha}^{*}, \widehat{e}_{\beta}^{*}\right)$ from $E_{1}^{*}$ holds (4.23), then $\widehat{e}^{*}$ also holds (4.25) and thus $\widehat{e}^{*} \in \widehat{\partial} \mu(\bar{e}) \cap E_{1}^{*}$. Note that

$$
\mathcal{J}_{u}^{\prime}\left(\bar{u}_{\bar{e}}, \bar{e}\right)=\varphi_{\bar{u}_{\bar{e}}, \bar{e}}=\widehat{e}_{\alpha}^{*}+\widehat{e}_{\beta}^{*} \quad \text { and } \quad \mathcal{J}_{e}^{\prime}\left(\bar{u}_{\bar{e}}, \bar{e}\right)=\left(\varphi_{\bar{u}_{\bar{e}}, \bar{e}}, y_{\bar{u}_{\bar{e}}+\bar{e}_{y}}, 0_{L^{\infty}(\Omega)^{*}}, 0_{L^{\infty}(\Omega)^{*}}\right) .
$$

Thus, by (4.23) we have

$$
\widehat{e}^{*}=\mathcal{J}_{e}^{\prime}\left(\bar{u}_{\bar{e}}, \bar{e}\right)+\left(0_{L^{2}(\Omega)}, 0_{L^{2}(\Omega)}, \widehat{e}_{\alpha}^{*}, \widehat{e}_{\beta}^{*}\right) .
$$

Using the local upper Hölderian selection $h(\cdot)$ of the solution map $S(\cdot)$ with $h(\bar{e})=\bar{u}_{\bar{e}}$ and $h(e)=\bar{u}_{e}$ for all $e \in \bar{B}_{\eta}(\bar{e}) \cap \operatorname{dom} \mathcal{G}$, from (4.33) we deduce that

$$
\begin{aligned}
\frac{\mu(e)-\mu(\bar{e})-\left\langle\widehat{e}^{*}, e-\bar{e}\right\rangle}{\|e-\bar{e}\|_{E}}= & \frac{\mathcal{J}\left(\bar{u}_{e}, e\right)-\mathcal{J}\left(\bar{u}_{\bar{e}}, \bar{e}\right)-\left\langle\widehat{e}^{*}, e-\bar{e}\right\rangle}{\|e-\bar{e}\|_{E}} \\
= & \frac{\mathcal{J}\left(\bar{u}_{e}, e\right)-\mathcal{J}\left(\bar{u}_{\bar{e}}, e\right)-\left\langle\widehat{e}_{\alpha}^{*}, e_{\alpha}-\bar{e}_{\alpha}\right\rangle-\left\langle\widehat{e}_{\beta}^{*}, e_{\beta}-\bar{e}_{\beta}\right\rangle}{\|e-\bar{e}\|_{E}} \\
& +\frac{\mathcal{J}\left(\bar{u}_{\bar{e}}, e\right)-\mathcal{J}\left(\bar{u}_{\bar{e}}, \bar{e}\right)-\left\langle\mathcal{J}_{e}^{\prime}\left(\bar{u}_{\bar{e}}, \bar{e}\right), e-\bar{e}\right\rangle}{\|e-\bar{e}\|_{E}} \\
= & \frac{\mathcal{J}\left(\bar{u}_{e}, e\right)-\mathcal{J}\left(\bar{u}_{\bar{e}}, e\right)-\left\langle\mathcal{J}_{u}^{\prime}\left(\bar{u}_{\bar{e}}, \bar{e}\right), \bar{u}_{e}-\bar{u}_{\bar{e}}\right\rangle}{\|e-\bar{e}\|_{E}} \\
& +\frac{\left\langle\widehat{e}_{\alpha}^{*}+\widehat{e}_{\beta}^{*}, \bar{u}_{e}-\bar{u}_{\bar{e}}\right\rangle-\left\langle\widehat{e}_{\alpha}^{*}, e_{\alpha}-\bar{e}_{\alpha}\right\rangle-\left\langle\widehat{e}_{\beta}^{*}, e_{\beta}-\bar{e}_{\beta}\right\rangle}{\| e-\bar{e}_{E}} \\
& +\frac{\mathcal{J}\left(\bar{u}_{\bar{e}}, e\right)-\mathcal{J}\left(\bar{u}_{\bar{e}}, \bar{e}\right)-\left\langle\mathcal{J}_{e}^{\prime}\left(\bar{u}_{\bar{e}}, \bar{e}\right), e-\bar{e}\right\rangle}{\|e-\bar{e}\|_{E}},
\end{aligned}
$$


and thus we have

$$
\begin{aligned}
& \frac{\mu(e)-\mu(\bar{e})-\left\langle\widehat{e}^{*}, e-\bar{e}\right\rangle}{\|e-\bar{e}\|_{E}}=\frac{\mathcal{J}\left(\bar{u}_{e}, e\right)-\mathcal{J}\left(\bar{u}_{\bar{e}}, e\right)-\left\langle\mathcal{J}_{u}^{\prime}\left(\bar{u}_{\bar{e}}, e\right), \bar{u}_{e}-\bar{u}_{\bar{e}}\right\rangle}{\|e-\bar{e}\|_{E}} \\
& +\frac{\left\langle\mathcal{J}_{u}^{\prime}\left(\bar{u}_{\bar{e}}, e\right)-\mathcal{J}_{u}^{\prime}\left(\bar{u}_{\bar{e}}, \bar{e}\right), \bar{u}_{e}-\bar{u}_{\bar{e}}\right\rangle}{\|e-\bar{e}\|_{E}} \\
& +\frac{\left\langle\widehat{e}_{\alpha}^{*}+\widehat{e}_{\beta}^{*}, \bar{u}_{e}-\bar{u}_{\bar{e}}\right\rangle-\left\langle\widehat{e}_{\alpha}^{*}, e_{\alpha}-\bar{e}_{\alpha}\right\rangle-\left\langle\widehat{e}_{\beta}^{*}, e_{\beta}-\bar{e}_{\beta}\right\rangle}{\|e-\bar{e}\|_{E}} \\
& +\frac{\mathcal{J}\left(\bar{u}_{\bar{e}}, e\right)-\mathcal{J}\left(\bar{u}_{\bar{e}}, \bar{e}\right)-\left\langle\mathcal{J}_{e}^{\prime}\left(\bar{u}_{\bar{e}}, \bar{e}\right), e-\bar{e}\right\rangle}{\|e-\bar{e}\|_{E}} \\
& =: \rho_{1}(e)+\rho_{2}(e)+\rho_{3}(e)+\rho_{4}(e) .
\end{aligned}
$$

By Lemma 4.9, we can find $\eta>0$ and $K_{M}>0$ such that the following inequality

$$
\left|\mathcal{J}_{u}^{\prime \prime}(u, e)\left(v_{1}, v_{2}\right)\right| \leq K_{M}\left\|z_{u, v_{1}}^{e}\right\|_{L^{2}(\Omega)}\left\|z_{u, v_{2}}^{e}\right\|_{L^{2}(\Omega)}
$$

holds for all $e \in B_{\eta}(\bar{e}), u \in \mathcal{U}_{a d}(e)$, and $v_{1}, v_{2} \in L^{2}(\Omega)$. By [23, Lemma 4.2], we get

$$
\left\|z_{u, v}^{e}\right\|_{L^{2}(\Omega)}=\left\|z_{u+e_{y}, v}\right\|_{L^{2}(\Omega)} \leq C_{3}\|v\|_{L^{1}(\Omega)}, \forall v \in L^{1}(\Omega)
$$

for some constant $C_{3}>0$ independent of $u$ and $e$. From this and (4.34) we infer that

$$
\left|\mathcal{J}_{u}^{\prime \prime}(u, e)\left(v_{1}, v_{2}\right)\right| \leq C\left\|v_{1}\right\|_{L^{1}(\Omega)}\left\|v_{2}\right\|_{L^{1}(\Omega)}, \forall v_{1}, v_{2} \in L^{2}(\Omega)
$$

where $C:=K_{M} C_{3}$. Using (4.35) and (4.24), we get

$$
\begin{aligned}
\lim _{e \rightarrow \bar{e}}\left|\rho_{1}(e)\right| & =\lim _{e \rightarrow \bar{e}} \frac{\left|\mathcal{J}\left(\bar{u}_{e}, e\right)-\mathcal{J}\left(\bar{u}_{\bar{e}}, e\right)-\left\langle\mathcal{J}_{u}^{\prime}\left(\bar{u}_{\bar{e}}, e\right), \bar{u}_{e}-\bar{u}_{\bar{e}}\right\rangle\right|}{\|e-\bar{e}\|_{E}} \\
& =\lim _{e \rightarrow \bar{e}} \frac{1}{2} \frac{\left|\mathcal{J}_{u}^{\prime \prime}\left(\widehat{u}_{e}, e\right)\left(\bar{u}_{e}-\bar{u}_{\bar{e}}\right)^{2}\right|}{\|e-\bar{e}\|_{E}} \leq \lim _{e \rightarrow \bar{e}} \frac{C\left\|\bar{u}_{e}-\bar{u}_{\bar{e}}\right\|_{L^{1}(\Omega)}^{2}}{2\|e-\bar{e}\|_{E}} \\
& =\lim _{e \rightarrow \bar{e}} \frac{C\|h(e)-h(\bar{e})\|_{L^{1}(\Omega)}^{2}}{2\|e-\bar{e}\|_{E}} \leq \lim _{e \rightarrow \bar{e}} \frac{C c^{2}\|e-\bar{e}\|_{E}^{2 æ}}{2\|e-\bar{e}\|_{E}}=0,
\end{aligned}
$$

where $\widehat{u}_{e}=\bar{u}_{\bar{e}}+\theta\left(\bar{u}_{e}-\bar{u}_{\bar{e}}\right)$ for some function $\theta(\cdot)$ with $0 \leq \theta(x) \leq 1$. In addition, by applying Lemma 4.11 we get for some constant $K_{M}>0$ that

$$
\begin{aligned}
\lim _{e \rightarrow \bar{e}}\left|\rho_{2}(e)\right| & =\lim _{e \rightarrow \bar{e}} \frac{\left|\left\langle\mathcal{J}_{u}^{\prime}\left(\bar{u}_{\bar{e}}, e\right)-\mathcal{J}_{u}^{\prime}\left(\bar{u}_{\bar{e}}, \bar{e}\right), \bar{u}_{e}-\bar{u}_{\bar{e}}\right\rangle\right|}{\|e-\bar{e}\|_{E}} \\
& \leq \lim _{e \rightarrow \bar{e}} \frac{\left\|\mathcal{J}_{u}^{\prime}\left(\bar{u}_{\bar{e}}, e\right)-\mathcal{J}_{u}^{\prime}\left(\bar{u}_{\bar{e}}, \bar{e}\right)\right\|_{L^{\infty}(\Omega)}\left\|\bar{u}_{e}-\bar{u}_{\bar{e}}\right\|_{L^{1}(\Omega)}}{\|e-\bar{e}\|_{E}} \\
& =\lim _{e \rightarrow \bar{e}} \frac{\left\|\varphi_{\bar{u}_{\bar{e}}, e}-\varphi_{\bar{u}_{\bar{e}}, \bar{e}}\right\|_{L^{\infty}(\Omega)}\left\|\bar{u}_{e}-\bar{u}_{\bar{e}}\right\|_{L^{1}(\Omega)}}{\|e-\bar{e}\|_{E}} \\
& \leq \lim _{e \rightarrow \bar{e}} \frac{K_{M}\|e-\bar{e}\|_{E}\left\|\bar{u}_{e}-\bar{u}_{\bar{e}}\right\|_{L^{1}(\Omega)}}{\|e-\bar{e}\|_{E}}=0 .
\end{aligned}
$$

Moreover, by denoting $\Omega_{i}=\Omega_{i}\left(\bar{e}, \bar{u}_{\bar{e}}\right)$ for $i=1,2,3$, it holds that $\left.\bar{u}_{\bar{e}}\right|_{\Omega_{1}}=\left.\alpha\right|_{\Omega_{1}}+\left.\bar{e}_{\alpha}\right|_{\Omega_{1}}$ and $\left.\bar{u}_{\bar{e}}\right|_{\Omega_{3}}=\left.\beta\right|_{\Omega_{3}}+\left.\bar{e}_{\beta}\right|_{\Omega_{3}}$ due to the definition of $\Omega_{1}$ and $\Omega_{3}$. Hence, we obtain

$$
\begin{aligned}
\rho_{3}(e) & =\frac{\left\langle\widehat{e}_{\alpha}^{*}+\widehat{e}_{\beta}^{*}, \bar{u}_{e}-\bar{u}_{\bar{e}}\right\rangle-\left\langle\widehat{e}_{\alpha}^{*}, e_{\alpha}-\bar{e}_{\alpha}\right\rangle-\left\langle\widehat{e}_{\beta}^{*}, e_{\beta}-\bar{e}_{\beta}\right\rangle}{\|e-\bar{e}\|_{E}} \\
& =\frac{\left\langle\left.\widehat{e}_{\alpha}^{*}\right|_{\Omega_{1}},\left.\bar{u}_{e}\right|_{\Omega_{1}}-\left.\bar{u}_{\bar{e}}\right|_{\Omega_{1}}-\left.e_{\alpha}\right|_{\Omega_{1}}+\left.\bar{e}_{\alpha}\right|_{\Omega_{1}}\right\rangle+\left\langle\left.\widehat{e}_{\beta}^{*}\right|_{\Omega_{3}},\left.\bar{u}_{e}\right|_{\Omega_{3}}-\left.\bar{u}_{\bar{e}}\right|_{\Omega_{3}}-\left.e_{\beta}\right|_{\Omega_{3}}+\left.\bar{e}_{\beta}\right|_{\Omega_{3}}\right\rangle}{\|e-\bar{e}\|_{E}} \\
& =\frac{\left\langle\left.\widehat{e}_{\alpha}^{*}\right|_{\Omega_{1}},\left.\bar{u}_{e}\right|_{\Omega_{1}}-\left.\alpha\right|_{\Omega_{1}}-\left.e_{\alpha}\right|_{\Omega_{1}}\right\rangle+\left\langle\left.\widehat{e}_{\beta}^{*}\right|_{\Omega_{3}},\left.\bar{u}_{e}\right|_{\Omega_{3}}-\left.\beta\right|_{\Omega_{3}}-\left.e_{\beta}\right|_{\Omega_{3}}\right\rangle}{\|e-\bar{e}\|_{E}} \\
& \geq 0 .
\end{aligned}
$$


Finally, since the map $e \mapsto \mathcal{J}(u, e)$ is Fréchet differentiable at $\bar{e}$ for each $u$, we get

$$
\lim _{e \rightarrow \bar{e}} \rho_{4}(e)=\lim _{e \rightarrow \bar{e}} \frac{\mathcal{J}\left(\bar{u}_{\bar{e}}, e\right)-\mathcal{J}\left(\bar{u}_{\bar{e}}, \bar{e}\right)-\left\langle\mathcal{J}_{e}^{\prime}\left(\bar{u}_{\bar{e}}, \bar{e}\right), e-\bar{e}\right\rangle}{\|e-\bar{e}\|_{E}}=0 .
$$

Summarizing the above we deduce that

$$
\liminf _{e \rightarrow \bar{e}} \frac{\mu(e)-\mu(\bar{e})-\left\langle\widehat{e}^{*}, e-\bar{e}\right\rangle}{\|e-\bar{e}\|_{E}}=\liminf _{e \rightarrow \bar{e}} \rho_{3}(e) \geq 0 .
$$

This implies that $\widehat{e}^{*} \in \widehat{\partial} \mu(\bar{e}) \cap E_{1}^{*}$.

From Theorem 4.15 and Theorem 4.17 we obtain the following characterization of regular subgradients of the marginal function $\mu(\cdot)$ in $E_{1}^{*}$.

Theorem 4.18. Let $\left(\bar{e}, \bar{u}_{\bar{e}}\right) \in \operatorname{gph} S$ and assume that all the assumptions of Theorem 4.15 hold, where (A4) holds with $æ>1 / 2$. Then, we have $\widehat{e}^{*}=\left(\widehat{e}_{y}^{*}, \widehat{e}_{J}^{*}, \widehat{e}_{\alpha}^{*}, \widehat{e}_{\beta}^{*}\right) \in \widehat{\partial} \mu(\bar{e}) \cap E_{1}^{*}$ if and only if $\widehat{e}^{*}=\left(\widehat{e}_{y}^{*}, \widehat{e}_{J}^{*}, \widehat{e}_{\alpha}^{*}, \widehat{e}_{\beta}^{*}\right) \in E_{1}^{*}$ satisfies

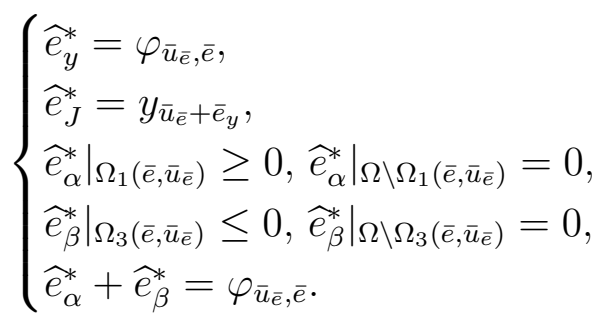

Proof. Applying Theorems 4.15 and 4.17, we obtain the assertion of the theorem.

Corollary 4.19. Let $\left(\bar{e}, \bar{u}_{\bar{e}}\right) \in \operatorname{gph} S$ and assume that all the assumptions of Corollary 4.16 hold, where (A4) holds with $æ>1 / 2$. Then, we have $\widehat{e}^{*}=\left(\widehat{e}_{y}^{*}, \widehat{e}_{J}^{*}, \widehat{e}_{\alpha}^{*}, \widehat{e}_{\beta}^{*}\right) \in \widehat{\partial} \mu(\bar{e}) \cap E_{1}^{*}$ if and only if $\widehat{e}^{*}=\left(\widehat{e}_{y}^{*}, \widehat{e}_{J}^{*}, \widehat{e}_{\alpha}^{*}, \widehat{e}_{\beta}^{*}\right) \in E_{1}^{*}$ satisfies (4.36).

Proof. It follows directly from Corollary 4.16 and Theorem 4.17.

Remark 4.20. If all the assumptions of Theorem 4.17 hold around $\bar{e} \in E$, then by using (2.12) and (2.13) we obtain the following lower estimates for the Mordukhovich and singular subdifferentials of $\mu(\cdot)$ via (4.23) as follows

$$
\partial \mu(\bar{e}) \supset \partial \mu(\bar{e}) \cap E_{1}^{*} \supset \underset{e{ }_{e} \operatorname{Limsup}_{\bar{e}}}{ }\left(\widehat{\partial} \mu(e) \cap E_{1}^{*}\right),
$$

and

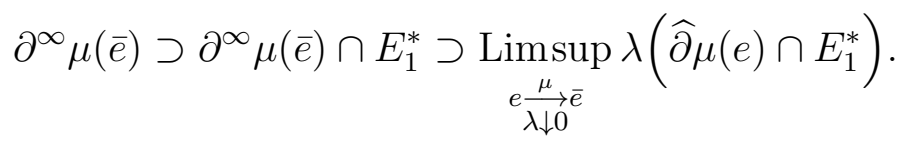

If, in addition, there exists a sequence $e_{n} \rightarrow \bar{e}$ with $\widehat{\partial} \mu\left(e_{n}\right) \cap E_{1}^{*} \neq \emptyset$, then $0 \in \partial^{\infty} \mu(\bar{e})$. Indeed, since $\widehat{\partial} \mu\left(e_{n}\right) \cap E_{1}^{*}$ is bounded by (4.23). Combining this with (4.38) yields $0 \in \partial^{\infty} \mu(\bar{e})$.

\section{Concluding remarks}

In this paper, we have obtained new results on differential stability of a class of optimal control problems of semilinear elliptic PDEs. We have established upper estimates for the regular, the Mordukhovich, and the singular subdifferentials of the marginal function $\mu(\cdot)$ in 
the setting that $E=L^{2}(\Omega)^{4}$ and $\mathcal{Q} \times \mathcal{U}_{a d}(e) \subset L^{2}(\Omega) \times L^{2}(\Omega)$. Furthermore, we have also obtained a new result on the existence of local upper Hölderian selections of the solution map $S(\cdot)$ as well as a lower estimate for the regular subdifferential of $\mu(\cdot)$ with respect to $E=L^{2}(\Omega)^{2} \times L^{\infty}(\Omega)^{2}$ and $\mathcal{Q}=L^{p_{0}}(\Omega)$, where $p_{0}>N / 2$. In the last setting, the problem of computing/estimating the limiting subdifferentials of $\mu(\cdot)$ is very complicated since the parametric space $E=L^{2}(\Omega)^{2} \times L^{\infty}(\Omega)^{2}$ is not Asplund. For this reason, such problem remains open.

Further investigation, we are also interested in the problem of computing subdifferentials of the marginal function $\mu(\cdot)$ with respect to the parametric space

$$
E=L^{2}(\Omega) \times L^{2}(\Omega) \times C(\bar{\Omega}) \times C(\bar{\Omega}) .
$$

Note that for the parametric space $E$ given by (5.1), subgradients of the marginal function $\mu(\cdot)$ will be in the form

$$
e^{*}=\left(e_{y}^{*}, e_{J}^{*}, e_{\alpha}^{*}, e_{\beta}^{*}\right) \in E^{*}=L^{2}(\Omega) \times L^{2}(\Omega) \times C(\bar{\Omega})^{*} \times C(\bar{\Omega})^{*},
$$

where $e_{\alpha}^{*}$ and $e_{\beta}^{*}$ are measures. We think that the problem of characterization of subgradients $e^{*}=\left(e_{y}^{*}, e_{J}^{*}, e_{\alpha}^{*}, e_{\beta}^{*}\right) \in E^{*}$ of $\mu(\cdot)$ with functions $e_{y}^{*}, e_{J}^{*}$ and measures $e_{\alpha}^{*}, e_{\beta}^{*}$ is an interesting and meaningful topic.

\section{References}

[1] D. T. V. An, N. T. ToAn, Differential stability of convex discrete optimal control problems, Acta Math. Vietnam., 43 (2018), pp. 201-217.

[2] D. T. V. An, J.-C. YaO, Further results on differential stability of convex optimization problems, J. Optim. Theory Appl., 170 (2016), pp. 28-42.

[3] D. T. V. An, N. D. Yen, Differential stability of convex optimization problems under inclusion constraints, Appl. Anal., 94 (2015), pp. 108-128.

[4] E. Asplund, Fréchet differentiability of convex functions, Acta Math., 121 (1968), pp. 31-47.

[5] J.-P. Aubin, Optima and Equilibria. An Introduction to Nonlinear Analysis, SpringerVerlag, Berlin, 1998.

[6] A. Auslender, Differentiable stability in nonconvex and nondifferentiable programming, Math. Programming Stud., 10 (1979), pp. 29-41.

[7] A. Auslender, M. Teboulle, Asymptotic Cones and Functions in Optimization and Variational Inequalities, Springer-Verlag, New York, 2003.

[8] J. F. Bonnans, A. Shapiro, Perturbation Analysis of Optimization Problems, Springer-Verlag, New York, 2000.

[9] E. CASAS, Second order analysis for bang-bang control problems of PDEs, SIAM J. Control Optim., 50 (2012), pp. 2355-2372.

[10] E. Casas, D. Wachsmuth, G. Wachsmuth, Sufficient second-order conditions for bang-bang control problems, SIAM J. Control Optim., 55 (2017), pp. 3066-3090. 
[11] N. H. Chieu, B. T. KIEn, N. T. ToAn, Further results on subgradients of the value function to a parametric optimal control problem, J. Optim. Theory Appl., 168 (2016), pp. $785-801$.

[12] A. L. Dontchev and R. T. Rockafellar, Implicit Functions and Solution Mappings, Springer, Dordrecht, 2009.

[13] P. H. Dien, N. D. Yen, On implicit function theorems for set-valued maps and their application to mathematical programming under inclusion constraints, Appl. Math. Optim., 24 (1991), pp. 35-54.

[14] J. Gauvin, F. Dubeau, Differential properties of the marginal function in mathematical programming, Math. Programming Stud., 19 (1982), pp. 101-119.

[15] J. Gauvin, F. Dubeau, Some examples and counterexamples for the stability analysis of nonlinear programming problems, Math. Programming Stud., 21 (1984), pp. 69-78.

[16] B. Gollan, On the marginal function in nonlinear programming, Math. Oper. Res., 9 (1984), pp. 208-221.

[17] A. D. Ioffe, V. M. Tinomirov, Theory of Extremal Problems, North-Holland, Amsterdam, 1979.

[18] B. S. Mordukhovich, Variational Analysis and Generalized Differentiation, I. Basic Theory, Springer-Verlag, Berlin, 2006.

[19] B. S. Mordukhovich, Variational Analysis and Generalized Differentiation, II. Applications, Springer-Verlag, Berlin, 2006.

[20] B. S. Mordukhovich, N. M. NAm, Variational stability and marginal functions via generalized differentiation, Math. Oper. Res., 30 (2005), pp. 800-816.

[21] B. S. Mordukhovich, N. M. Nam, N. D. Yen, Subgradients of marginal functions in parametric mathematical programming, Math. Program., Ser. B, 116 (2009), pp. 369396.

[22] R. R. Phelps, Convex Functions, Monotone Operators and Differentiability, Second Edition, Springer-Verlag, Berlin, 1993.

[23] N. T. Qui, D. Wachsmuth, Stability for bang-bang control problems of partial differential equations, Preprint, (2017), pp. 1-20.

[24] S. M. Robinson, Generalized equations and their solutions. I. Basic theory, Math. Programming Stud., 10 (1979), pp. 128-141.

[25] R. T. RockAfellar, Lagrange multipliers and subderivatives of optimal value functions in nonlinear programming, Math. Programming Stud., 17 (1982), pp. 28-66.

[26] R. T. Rockafellar, R. J.-B. Wets, Variational Analysis, Springer-Verlag, Berlin, 1998.

[27] L. ThiBault, On subdifferentials of optimal value functions, SIAM J. Control Optim., 29 (1991), pp. 1019-1036.

[28] N. T. TOAn, Mordukhovich subgradients of the value function in a parametric optimal control problem, Taiwanese J. Math., 19 (2015), pp. 1051-1072. 
[29] N. T. TOAn, B. T. KIEN, Subgradients of the value function to a parametric optimal control problem, Set-Valued Var. Anal., 18 (2010), pp. 183-203.

[30] L. Q. Thuy, N. T. Toan, Subgradients of the value function in a parametric convex optimal control problem, J. Optim. Theory Appl., 170 (2016), pp. 43-64.

[31] F. Tröltzsch, Optimal Control of Partial Differential Equations. Theory, Methods and Applications, American Mathematical Society, Providence, RI, 2010.

[32] A. Visintin, Strong convergence results related to strict convexity, Comm. Partial Differential Equations, 9 (1984), pp. 439-466. 